\title{
EFFECT OF CURING PARAMETERS AND CONFIGURATION ON THE EFFICACY OF ULTRAVIOLET LIGHT CURING SELF-ADHESIVE MASKS USED FOR ABRASIVE JET MICRO-MACHINING AND MICROFLUIDIC DEVICE FABRICATION
}

by

Farbod Ahmadzadeh

Bachelor of Mechanical Engineering, Ryerson University, Toronto, Canada 2014

\author{
A thesis \\ presented to Ryerson University \\ in partial fulfillment of the \\ requirement for the degree of \\ Master of Applied Science \\ in the Program of \\ Mechanical and Industrial Engineering \\ Toronto, Ontario, Canada, 2017 \\ (C) Farbod Ahmadzadeh 2017
}




\section{AUTHOR'S DECLARATION FOR ELECTRONIC SUBMISSION OF THESIS}

I hereby declare that I am the sole author of this thesis. This is a true copy of the thesis, including any required final revisions, as accepted by my examiners.

I authorize Ryerson University to lend this thesis to other institutions or individuals for the purpose of scholarly research.

I further authorize Ryerson University to reproduce this thesis by photocopying or by other means, in total or in part, at the request of other institutions or individuals for the purpose of scholarly research.

I understand that my thesis may be made electronically available to the public. 


\begin{abstract}
Effect of curing parameters and configuration on the efficacy of ultraviolet light curing self-adhesive masks used for abrasive jet micro-machining and Microfluidics device fabrication

Farbod Ahmadzadeh

Master of Applied Science, 2017

Mechanical and Industrial Engineering

Ryerson University
\end{abstract}

Soft lithography techniques has been used widely in the past decade to fabricate microfluidic chips used in biomedical applications. Abrasive jet machining (AJM) has been used to fabricate similar chips using particle erosion mechanisms. This thesis proposes a new technique using a UV light sensitive self-adhesive mask (RapidMask) and AJM to fabricate a three dimensional flow focusing microfluidic chip where the depth of the channel is allowed to vary along the channel length.

A detailed characterization of the effect of curing parameters of a UV light curing selfadhesive mask on the resulting feature resolution is reported. Instead of relying on the manufacturer recommended curing parameters which were vaguely described for specific UV curing units, it was found that measured energy density could be used to quantify a recommended cure that is independent of the curing unit. The best achievable pattern on borosilicate glass using $\mathrm{RM}$ and $\mathrm{AJM}$ was found and reported along with the erosion rates of uncured, cured RM during AJM. A new methodology was introduced to use multiple layers of the RM in order to increase the achievable feature aspect ratio.

The results of the RM curing and multiple layer investigation were then used to fabricate a three dimensional flow focusing chip with a varying depth at the focusing junction. The chip was then sealed and tested to demonstrate its capabilities and potential in healthcare and biomedical applications. To the best knowledge of the author, this thesis is the first to report using a double layer RM to fabricate a microfluidic chip using AJM. 


\section{Acknowledgements}

I would like to thank:

* Dr. Marcello Papini for providing me with the opportunity of joining his research team and his persistent and friendly support, encouragement, patience and advices in all steps of my MASc.

* Dr. Scott Tsai, for giving me the opportunity to work in the field of microfluidics and for his encouragements, support, and guidance throughout the course of this research project.

* The financial support of the Natural Sciences and Engineering Research Council of Canada (NSERC) and the Canada Research Chairs Program.

Ryerson University for the fantastic facilities and equipment provided for this research.

Alan Machin, Qiang Li, Chao Ma, Grace He, Joseph Amankrah, Andrew Heim, and Roy Churaman for their technical support.

My good friends Dr. Vahid Hadavi, Dr. Naser Haghbin, Dr. Mohammad Reza Sookhaklari, Ali Nouhi, Vaskar Gnyawali, Stephanie Buryk, and Morteza Jeyhani for their valuable consultations. 


\section{Dedication}

This thesis is dedicated to my beloved parents, my dear brother Farivar Ahmadzadeh, and all my loved ones. 


\section{Table of Contents}

AUTHOR'S DECLARATION FOR ELECTRONIC SUBMISSION OF THESIS ..................... ii

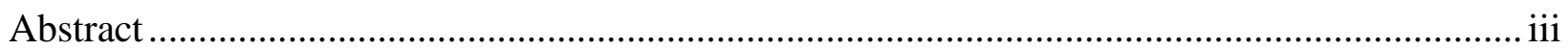

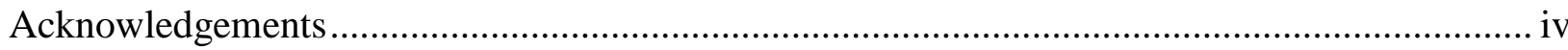

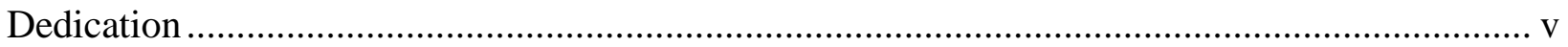

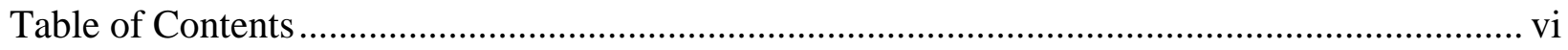

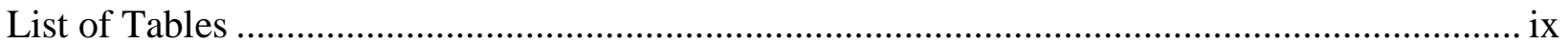

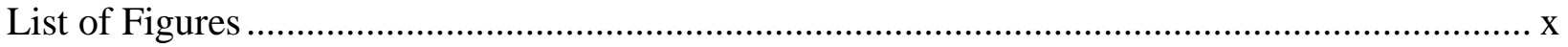

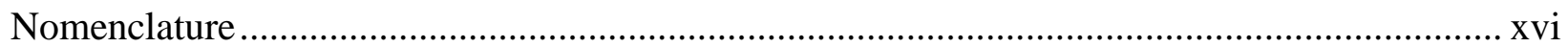

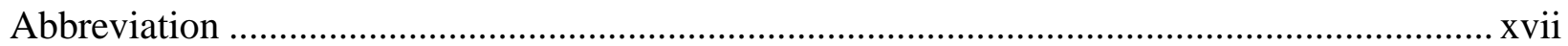

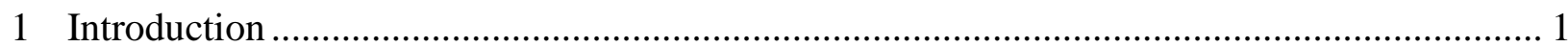

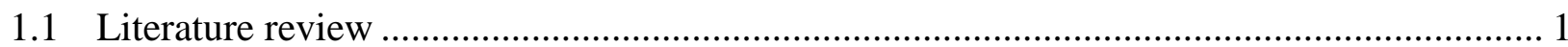

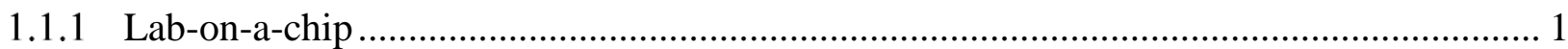

1.1.2 Abrasive jet micromachining ............................................................................ 5

1.1.3 AJM vs. traditional micro-manufacturing methods ............................................... 7

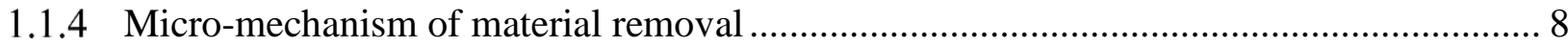

1.1.5 Effect of process parameters on solid particle erosion.......................................... 11

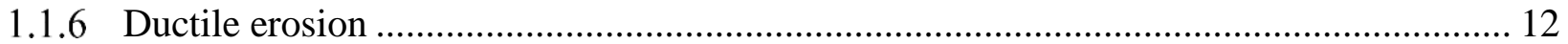

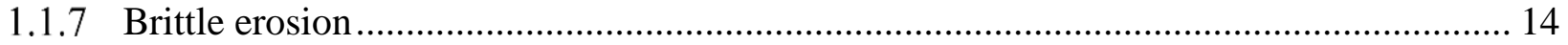

1.1.8 Microfluidic device fabrication using AJM ....................................................... 20 


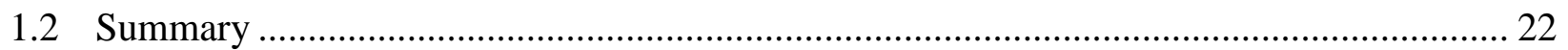

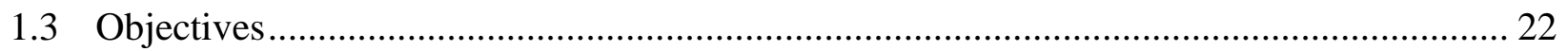

2 Effect of curing parameters and configuration on the efficacy of ultraviolet light curing selfadhesive masks used for abrasive jet micro-machining.............................................................. 24

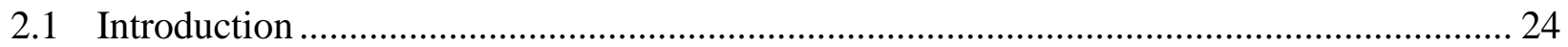

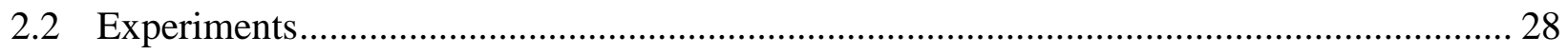

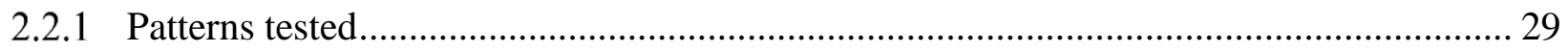

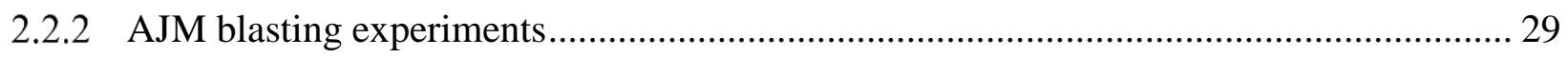

2.2.3 Effect of UV curing unit geometry and energy dose ................................................... 31

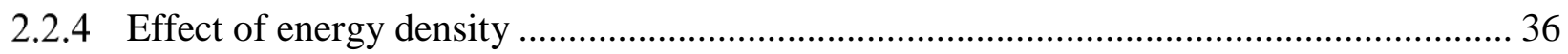

2.2.5 Effect of pattern orientation and location....................................................................... 37

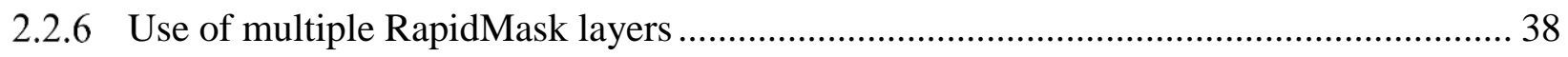

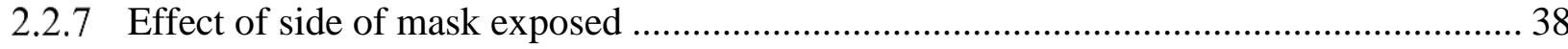

2.2.8 Erosion rate of uncured, cured RM, and borosilicate glass ............................................. 39

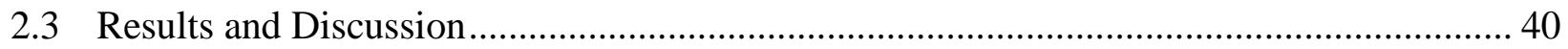

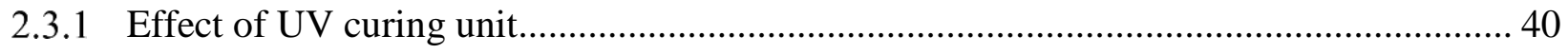

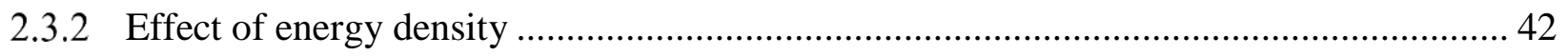

2.3.3 Effects of pattern orientation and location within cylindrical unit................................... 46

2.3.4 Effect of multiple RapidMask layers …………….................................................... 48

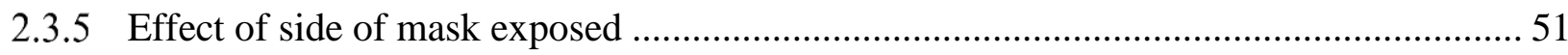

2.3.6 Erosion rate of Uncured RM, cured RM, and borosilicate glass.................................... 52 


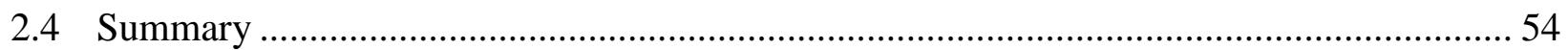

3 Application of AJM microfabrication to microfluidic three dimensional flow focusing ....... 56

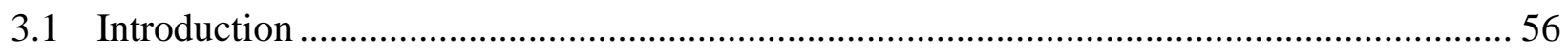

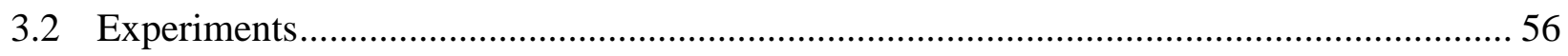

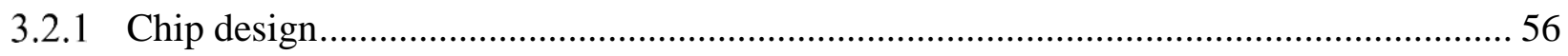

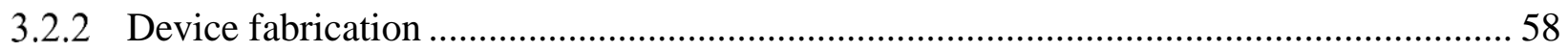

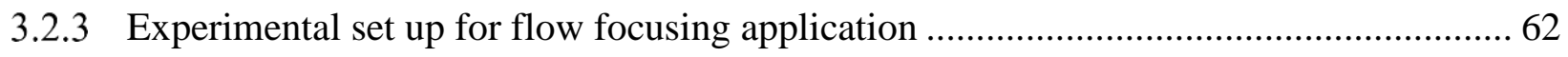

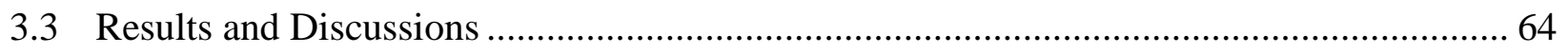

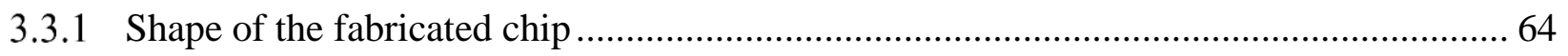

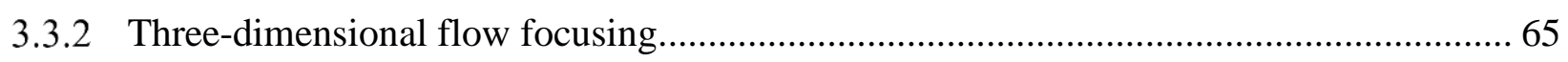

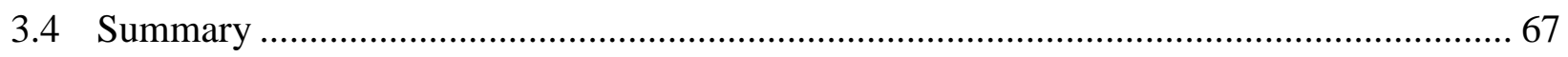

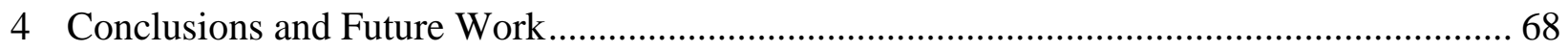

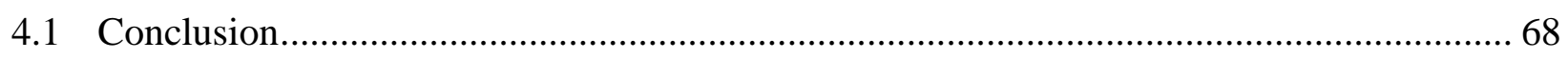

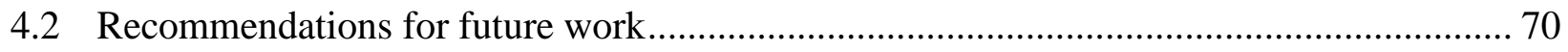

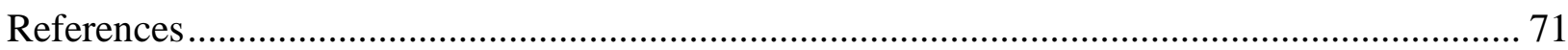




\section{List of Tables}

Table 1 - UV light energy densities resulting from curing in the cylindrical Letralite unit for the manufacturer recommended exposure times for HT RM .................................................... 36 


\section{List of Figures}

Figure 1.1 - Soft lithography fabrication process flow of a 3D flow focusing device. (a) Si wafer substrate. (b) Formation of double-step and single-step SU-8 mold. (c) Pouring of PDMS. (d) Removing PDMS from the mold. (e) Bonding of two layers via manual alignment and (f) isometric

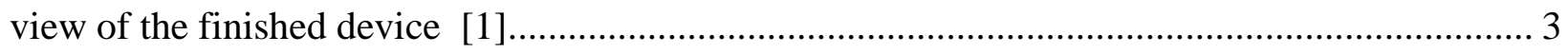
Figure 1.2 - A complex microchannel system. (A) representations of a top and bottom masters and resulting channels.(B) Optical micrograph of the channel system on PDMS membrane Optical micrograph. (C) Optical micrograph of channels with fluid in them (D) SEM image of the microstructure made of epoxy polymer that was cured under an ultraviolet light and dissolving the

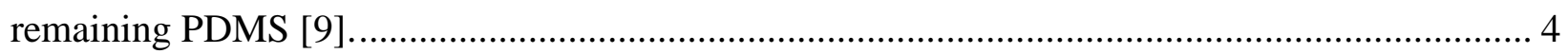

Figure 1.3 - Wax-printing-based technique of fabricating three dimensional microfluidic channels

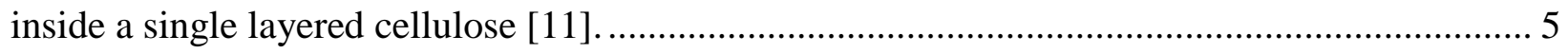

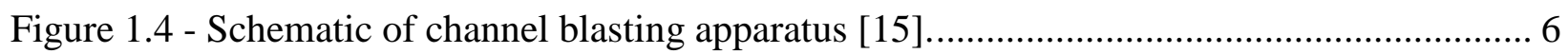

Figure 1.5 - Schematic of the variation of erosion with impact angle: (a) for ductile materials; (b)

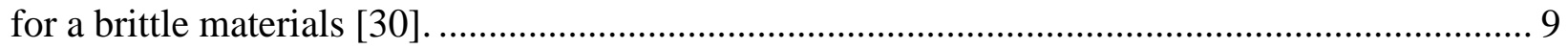

Figure 1.6 - Mechanisms of material removal by solid particle impact, (a) micro-cutting, (b)

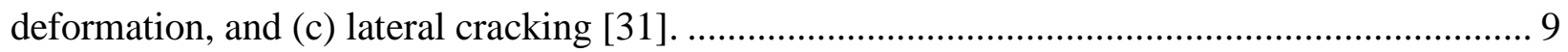

Figure 1.7 - Target material displacement and removal during continuous particles impact [41].

Figure 1.8 - Material removal mechanism when abrasive impact tangentially or scratching abrasive

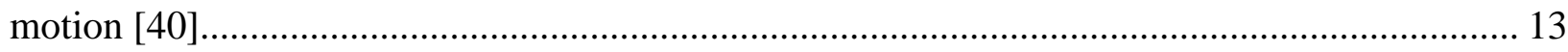

Figure 1.9 - Schematic of brittle erosion caused by cracking and fragmentation of surface material [40]. 15

Figure 1.10 - Crater shape \& Crack propagation in brittle material [42]. 15 
Figure 1.11 - SEM photograph of a lateral crack formation on a borosilicate glass target surface [43]. 16

Figure 1.12 - Crater impressions are explained with elastic-plastic material theory [45].......... 17

Figure 1.13 - scratch impressions are explained with elastic-plastic material theory [45].......... 18

Figure 1.14 - Schematic of a crater impression showing cleavage fracture on a brittle material [45].

Figure 1.15 - Schematic of two material removal modes: (1) scratching at the contact area (2) network cracking at its neighborhood [46]...... 19

Figure 1.16 - Capillary electrophoresis chip fabricated by AJM with PDMS inlets/outlets glued [47].

Figure 1.17 - Schematic of AJM apparatus to fabricate micro-channels using sacrificial masks [48].

Figure 2.1 - Procedure to cure the RM using a patterned transparency that allows UV light from the source to pass through the transparent patterns of the photomask. The UV light is transferred onto the photosensitive RM film..... 27

Figure 2.2 - Procedure to etch the surface of the glass. The patterned RM is attached to the glass, and AJM is used to transfer the pattern to the glass substrate. 27

Figure 2.3 - Microscopic images of cured and uncured regions of RM after exposure to the UV unit and being eroded by AJM up to the circled region. 28

Figure 2.4 - Image of (a) negative photomask with pattern design used and (b) exposed patterned $\mathrm{RM}$ with distance of $\sim 1.5-2 \mathrm{~mm}$ between the channel patterns. 29

Figure 2.5 - Schematic of abrasive jet micromachining experimental apparatus [65].............. 30 Figure 2.6 - Cylindrical ultraviolet exposure unit. 32 
Figure 2.7 - Schematic of the mini conveyor UV exposure unit in (a) isometric view, (b) sectioned isometric view showing the placement of the samples and their distance from the lamp. The system

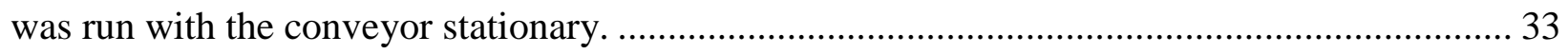

Figure 2.8 - Schematic of UV meter used to measure the UV radiation intensities. ................. 34 Figure 2.9 - Schematic of the cylindrical exposure unit in (a) side view, showing the placement of the transparent removable cover, and in (b) front view, showing the measured intensities along the length of the cylinder. 34

Figure 2.10 -Schematic of the mini conveyor unit showing the UV intensity and its region, shown in dotted lines with dimensions. The regions outside of the dotted areas are not exposed to UV light. 35

Figure 2.11 - Two sets of samples, with each box representing patterns of 250, 300, 400, 500, and $600 \mu \mathrm{m}$ wide channels, placed in the cylindrical UV curing system (Fig. 2.9) with channel length: (a) parallel and perpendicular to the cylinder $(x)$ axis; (b) perpendicular to the cylinder at different locations along the cylinder length. The curing lamp is parallel to the $x$ axis (Fig. 2.9(b)). The intensities are approximately constant within the zones shown within the dashed lines. 37 Figure $2.12-$ Intensity measurement, in $\mathrm{mW} / \mathrm{cm}^{2}$, of the cylindrical and mini-conveyor exposure units at various $y$ distances from the source. The position of the mask during curing in each unit is indicated. 40

Figure 2.13 - Measured widths of cured HT RM and AJM channels for 5 different design widths using the mini-conveyor curing system and cylindrical UV unit at the same energy density of 516 $\mathrm{mJ} / \mathrm{cm}^{2}$. Error bars indicate the standard deviation of the results of 6 sets of experiments for the UV curing process and 3 sets of experiments for the AJM process. 
Figure 2.14 - Measured widths of HT RM cured at energy densities of 258, 516, 774, 1032, and $1548 \mathrm{~mJ} / \mathrm{cm}^{2}$. Error bars indicate one standard deviation of 4 sets of experiments. 43

Figure 2.15 - Measured widths of HD RM cured at energy densities of 258, 387, 516, 774, 1032, and $1548 \mathrm{~mJ} / \mathrm{cm}^{2}$. Error bars indicate one standard deviation of 4 sets of experiments.......... 44

Figure 2.16 - Measured widths of cured HT RM and resulting AJM channel widths when curing at three energy densities. Error bars indicate one standard deviation of for 4 sets of experiments for UV exposure and 3 sets of AJM process. 45

Figure 2.17 - Measured widths of cured HD RM and resulting AJM channel widths when curing at two energy densities. Error bars indicate one standard deviation of for 4 sets of experiments for UV exposure and 3 sets of AJM process. 46

Figure 2.18 - Effect of channel orientation relative to curing cylinder axis. Measured width of cured region of HT RM for 5 different channel widths exposed in cylindrical unit at energy density $516 \mathrm{~mJ} / \mathrm{cm}^{2}$. The samples were placed perpendicular and parallel to the axis of the cylinder as in Fig. 2.11a. Error bars indicate one standard deviation of 4 sets of experiments.

Figure 2.19 - Measured widths of cured HT RM and resulting AJM channel widths cured at two energy densities, 384 and $516 \mathrm{~mJ} / \mathrm{cm}^{2}$. The samples were placed with orientations shown in Fig. 2.11b. Error bars indicate one standard deviation from 4 sets of experiments for UV exposure and

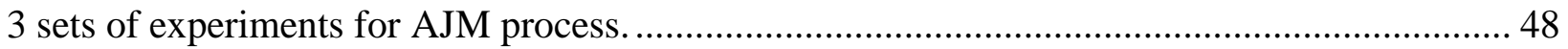
Figure 2.20 - Measured widths of cured single and double HT RM and resulting AJM channel widths exposed in the cylindrical UV exposure unit at an energy density of $516 \mathrm{~mJ} / \mathrm{cm}^{2}$. Error bars indicate one standard deviation of 4 sets of experiments for UV exposure and 3 sets of AJM process. 50 
Figure 2.21 - Centerline depth of AJM channels machined using single and double HT RM for 1 and 5 AJM passes on the glass. Error bars indicate one standard deviation from 4 measurements of eroded channels. 51

Figure 2.22 - Effect of curing the RM from dull and shiny sides. Measured width of cured region and resulting AJM channel widths of HT RM for 5 different channel widths exposed in cylindrical unit at UV energy density $516 \mathrm{~mJ} / \mathrm{cm}^{2}$. The samples were exposed from the dull and shiny side and were placed with set 1 orientation shown in Fig. 2.11b. Error bars indicate one standard deviation of 4 sets of experiments for UV exposure and 3 sets of AJM process. 52 Figure 2.23- Normalized erosion rate of uncured, UV cured RM, and borosilicate glass as a function of angle of impingement. 90 indicates nozzle angle with respect to the RM surface. The curves were normalized to their maximum erosion rates of $0.035,0.014$, and $0.116 \mathrm{~mm}^{3} / \mathrm{g}$, for the UV cured RM, uncured RM, and glass, respectively. Error bars indicate the normalized standard deviation of 3 sets of experiments at each angle. 53 Figure 3.1- Schematic of the chip pattern design with $250 \mu \mathrm{m}$ channel width for all inlets channels and depth change at the junction. 57 Figure 3.2 - 3D Schematic of the device as sample flow is focused in $y$ - $z$ plane by two sheath flows. 58

Figure 3.3- Schematic of (a) design layout (b) patterned transparency and (c) cured two layers HD RM used to fabricate the three dimensional flow focusing device. 59 Figure 3.4 - Schematic of the procedure to etch the surface of the glass. The patterned RM is attached to the glass, and AJM is used to transfer the pattern to the glass substrate. Schematic is not scale. 60 
Figure 3.5 - Schematic of microfluidic chip fabricated on a borosilicate glass and PDMS layer on top. 61

Figure 3.6 - A representative image of the inverted microscope set up used to image the flow focusing experiments [71] 62 Figure 3.7 - A representative image of confocal imaging microscopy set up used to image the flow focusing experiments. 63 Figure 3.8 - Profile of channels B, C, D in Fig. 3.1 of the chip fabricated by AJM on a borosilicate substrate. 64

Figure 3.9 - Measured width of cured RM and AJM channels of A, B, C, D channels, Fig. 3.1, shown in primary axis with measured depth of the channels shown on the secondary axis. The error bars indicate one standard deviation of 3 measurements of RM and eroded channels....... 65 Figure 3.10 - An image of top view of a focused flow in the microfluidic chip fabricated with AJM using RM. The flow rate was set at $6 \mu \mathrm{L} / \mathrm{min}$ and $100 \mu \mathrm{L} / \mathrm{min}$ for sample and two sheath flows, respectively. 66

Figure 3.11 - The confocal imaging of the channel at the focusing junction. The flow rate was set at $6 \mu \mathrm{L} / \mathrm{min}$ and $100 \mu \mathrm{L} / \mathrm{min}$ for sample flow rate and two sheath flows, respectively. 67 


\section{Nomenclature}

$\begin{array}{lc}d & \text { Particle diameter }(\mu \mathrm{m}) \\ E & \text { Young's modulus }(\mathrm{GPa}) \\ H & \text { Material hardness }(\mathrm{GPa}) \\ K & \text { Facture toughness }\left(\mathrm{J} / \mathrm{cm}^{2}\right) \\ m_{p} & \text { Particle mass }(\mathrm{g}) \\ v & \text { Particle velocity }(\mathrm{m} / \mathrm{s}) \\ v_{n}, v_{t} & \text { Normal and tangential threshold velocities }(\mathrm{m} / \mathrm{s}) \\ \theta & \text { Angle of impact } \\ \rho_{p} & \text { Particle density }\left(\mathrm{kg} / \mathrm{m}^{3}\right)\end{array}$




\section{Abbreviation}

$\begin{array}{lc}\text { AJM } & \begin{array}{c}\text { Abrasive jet micromachining } \\ \text { CAC }\end{array} \\ \text { EDM } & \text { Collagen-alginate composite } \\ \text { HD } & \text { High Detail } \\ \text { HT } & \text { High Tack } \\ \text { MEMS } & \text { Micro-Electromechanical systems } \\ \text { PDMS } & \text { Polydimethylsiloxane } \\ \text { RM } & \text { RapidMask } \\ \text { SEM } & \text { Scanning Electron Microscopy }\end{array}$




\section{Introduction}

This chapter presents a broad overview of the literature relevant to the micro-fabrication on microfluidic devices using abrasive jet micro-machining (AJM). It is meant to provide background and motivation for the objectives of the thesis.

\subsection{Literature review}

\subsubsection{Lab-on-a-chip}

Microfluidics has a growing role in biological and healthcare applications. Its capability for analysis of biological samples such as biomolecules, cells and functionalized beads has revolutionized conventional assay techniques [1]. Micro-fabrication has been a key to the development of microfluidics devices. Van Dijke et al. [2] stated that micro-fabrication has provided a platform for the development of finer and more accurate features. Such precision has resulted in increased reliability and capability of analyses including, but not limited to, the replication of biological environments such as a heart artery.

Flow confinement in microfluidics has been an essential feature in the study of biological samples as well as in biomedical research. When studying biological samples in micro-channels, the position of moving micro particles is optimal when positioned in the center of the channel. If micro particles deviate from this position, the results obtained may be compromised. For example, Chiu et al. [1] stated that to minimize channel clogging and sample fouling in the channel, it is essential to keep the sample flow near the center of the channel. A three-dimensional flow focusing microfluidic geometry can focus microparticles to the center of the microchannel cross section. Flow cytometry is one of methods used in clinical applications such as cell counting. Roederer et al. [3] showed that using flow cytometry, valuable studies can be conducted on cells and particles of different sizes with various properties. Microfluidic flow cytometry systems hold a clinical role in the diagnosis and monitoring of life threatening diseases such as AIDS and leukemia [4]. As such, improvements in current microfluidic flow cytometry systems are highly desired [5]. The advantages of using microfluidic devices instead of conventional flow chambers for flow cytometers include, but are not limited, cost reduction, environment size reduction where samples are analyzed, a decrease in cross contamination, and a decrease in volume of reagents and wastes [6]. 
Microfluidic devices are commonly termed "lab-on-chip" devices as a result of their many functions. There are several components common to lab-on-chip devices including pumps, dispensers, and separators. These components allow for multiple experiments to be conducted using a single platform [2]. Implementation of multiple components located on one device, by replicating biological environments, makes lab-on-chip devices well suited for applications in clinical research. For instance, Jun et al. [7] stated that microfluidic chips are used for production of collagen-alginate composite (CAC) fibers as an alternative to melt-spinning because the high temperature used during the process stops cell capsulations within the fibers. Although lab-on-chip devices hold many advantages over their conventional counterparts, there are still opportunities for further enhancement and improvements of the devices' capabilities.

Lab-on-chip capabilities can be drastically enhanced by including the ability to control fluid flow in the third dimension. Three-dimensional microstructures with exceptional geometries and features have created avenues in various microfluidics applications in healthcare related studies and research, [8]. There have been a number of methods traditionally used to fabricate 3D focusing devices. Chiu et al. [1] demonstrated a novel method to create three dimensional flow focusing of microchannels using photolithography, Fig 1.1. In this study, a device was fabricated consisting of two separate components, top and bottom layers. Both parts are made of polydimethylsiloxane (PDMS, Sylgard 184, Dow Corning, MI). Desired patterns were printed on each part using photolithography and soft lithography processes. SU-8 was used as a mold with a specific design and uncured PDMS was poured on it. The microfluidic device was then cured at $65^{\circ} \mathrm{C}$ in order to cure the viscous PDMS to its solid form. Finally, the two parts were carefully and precisely bonded, Fig. 1.1f. 


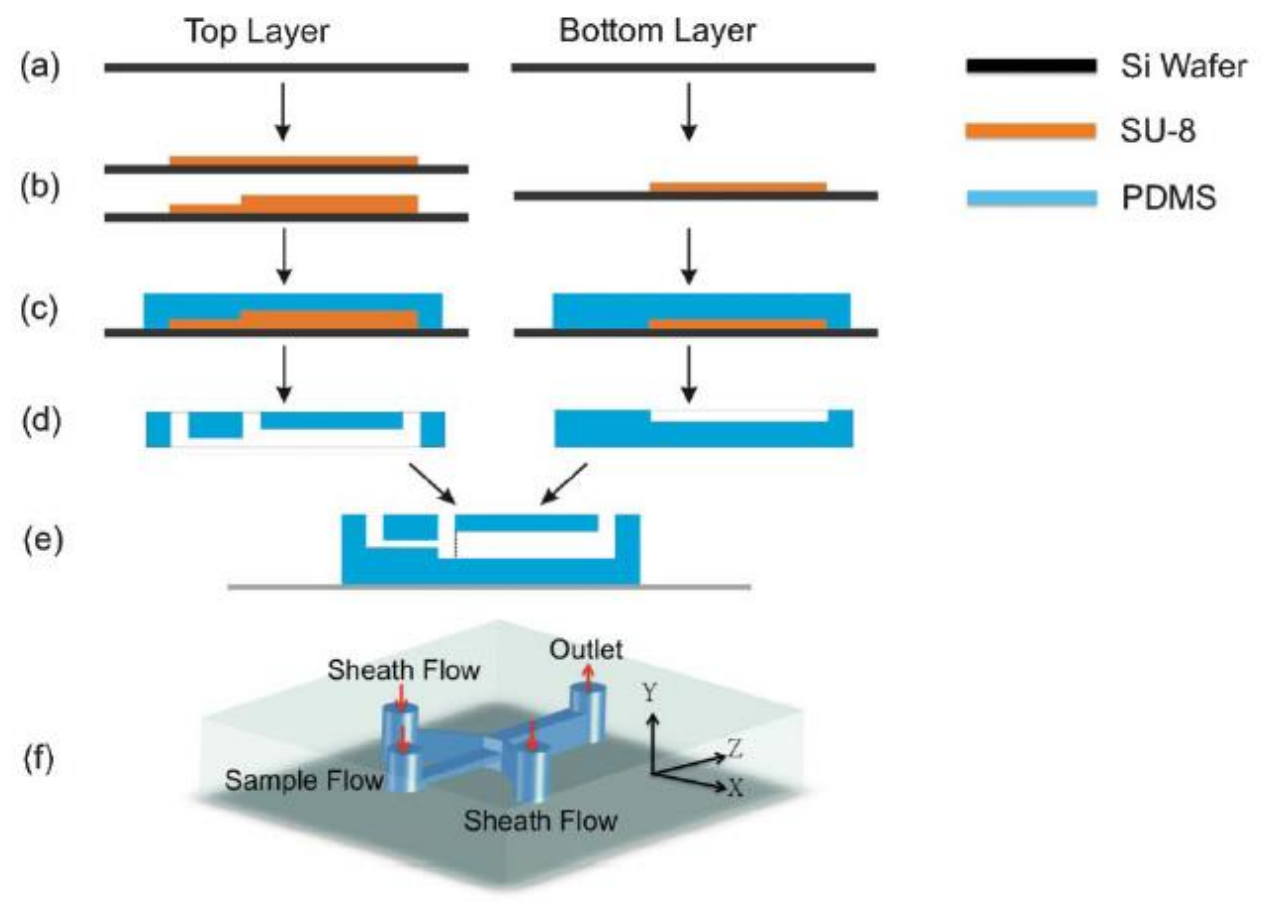

Figure 1.1 - Soft lithography fabrication process flow of a 3D flow focusing device. (a) Si wafer substrate. (b) Formation of double-step and single-step SU-8 mold. (c) Pouring of PDMS. (d)

Removing PDMS from the mold. (e) Bonding of two layers via manual alignment and (f) isometric view of the finished device [1].

The method explained is one of the well-studied techniques used to fabricate microfluidic devices. Soft lithography using SU-8 and PDMS is relatively cheap compared to other microfabrication techniques such as ion beam and electron beam lithography. However, one of the main drawback of this technique is the need for clean rooms. Clean rooms are expensive to maintain and operate which can affect this process in the long run in terms of cost and commercialization.

J.R Anderson et al. [9] showed a procedure to create a unique 3D microchannel using PDMS. The device consisted of channels on each face of a membrane. Two level photolithography and molding were used to create two masters. The two masters were aligned with uncured PDMS between the two masters, and then the entire assembly was cured thermally. After curing, the masters were removed in order and a thin PDMS membrane were interconnected (Fig. 1.2). The resulting product was a complex micro structure with microchannels at different levels in the $z$ direction, Fig. 1.2. 


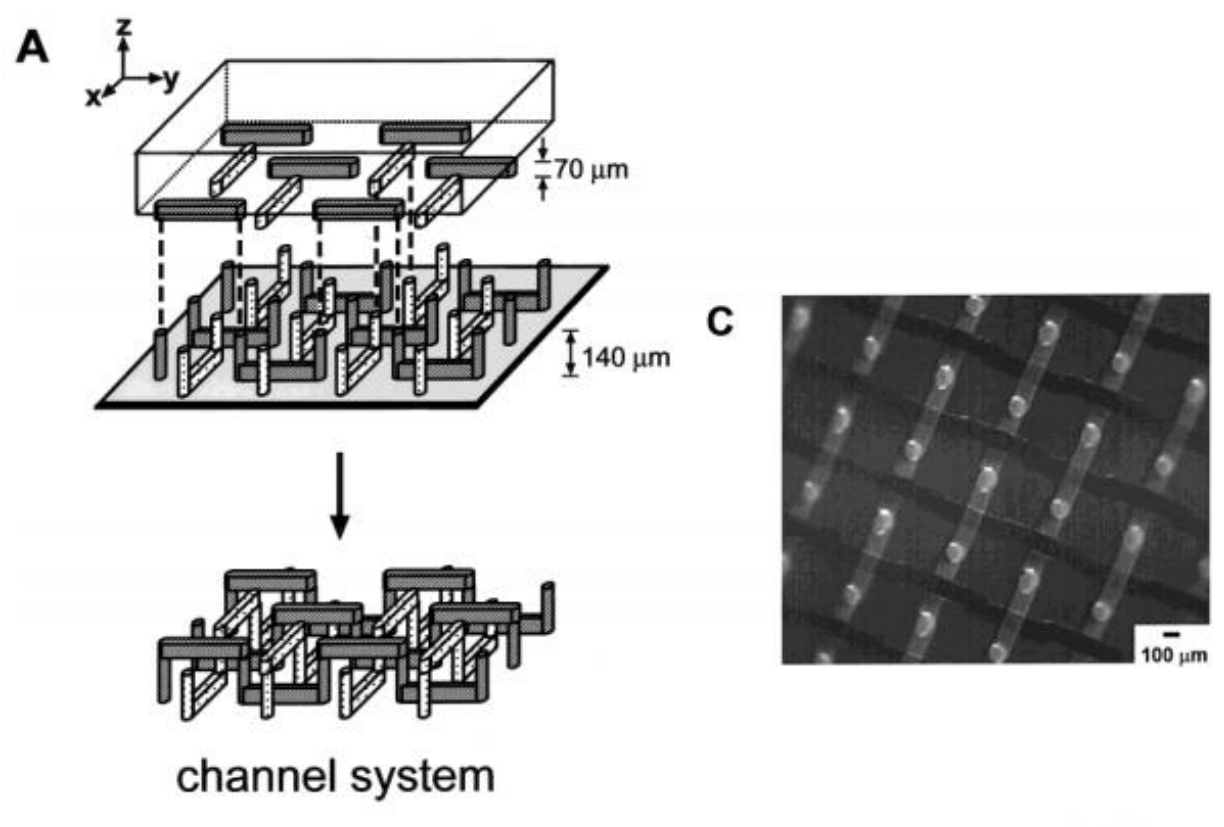

B
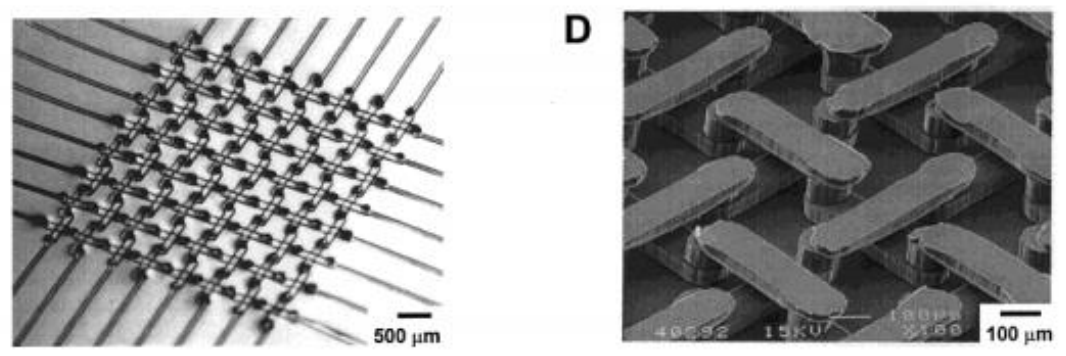

Figure 1.2 - A complex microchannel system. (A) representations of a top and bottom masters and resulting channels.(B) Optical micrograph of the channel system on PDMS membrane Optical micrograph. (C) Optical micrograph of channels with fluid in them (D) SEM image of the microstructure made of epoxy polymer that was cured under an ultraviolet light and dissolving the remaining PDMS [9].

For devices fabricated with methods explained above, the surface roughness and waviness of the channels may vary. Moreover, traditional fabrication technologies are limited to twodimensional (2D) fabrication, unless complex and expensive procedures are introduced [10]. Nevertheless, there have been previous studies that produce three-dimensional microfluidic devices using alternative methods. Li and Liu [11] fabricated a three-dimensional microfluidic device using wax-printing-based techniques (Fig. 1.3) while Martinez et al. [12] demonstrated fabricating devices in layered paper and tape for environmental monitoring and water analysis. 


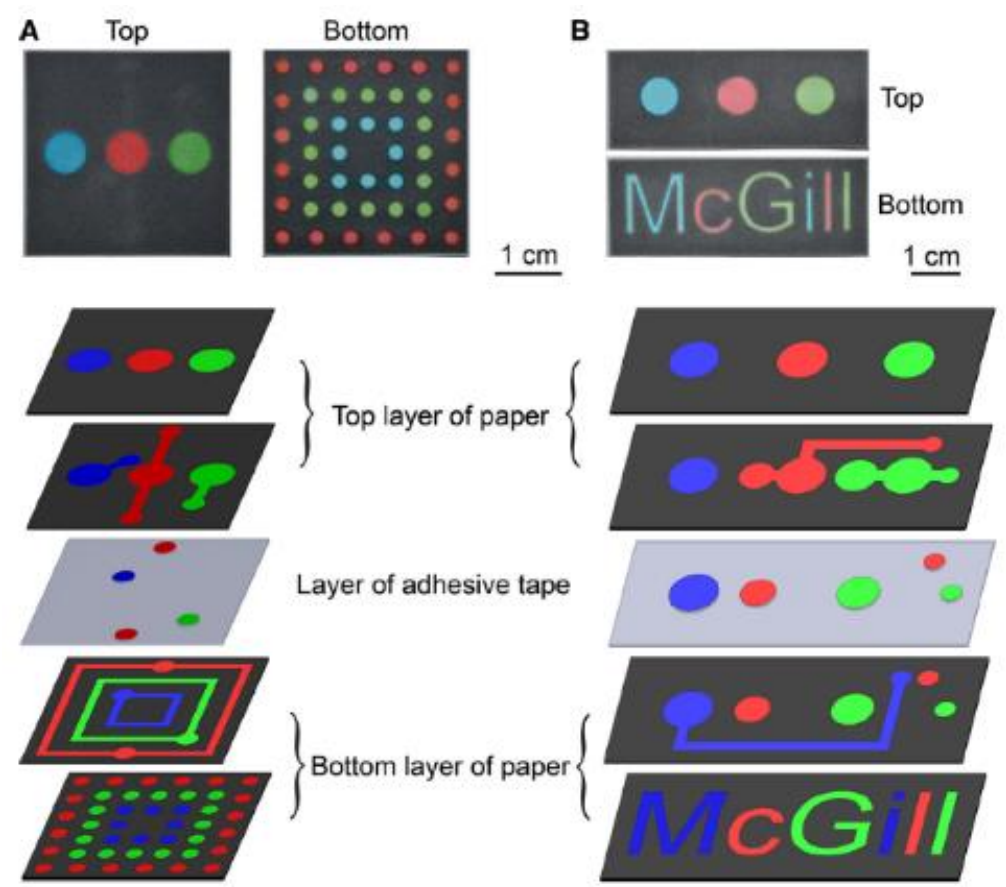

Figure 1.3 - Wax-printing-based technique of fabricating three dimensional microfluidic channels inside a single layered cellulose [11].

\subsubsection{Abrasive jet micromachining}

Abrasive Jet micro-machining, AJM, is a novel and developing method for the fabrication and forming of micro channels, micro holes, and 3D features. In the AJM technique, abrasive particles are accelerated by compressed air and directed toward a surface target through a nozzle, Fig. 1.4. The micro particles impact the substrate, ductile or brittle, with high velocities at a defined angle of strike and remove target material through erosion. Material removal occurs due to the superposition of the material removed by many particle impacts, [13]. Many investigations have been conducted regarding the advantages of AJM fabrication such as effective material removal mechanisms, the influences of material properties, input parameters on the erosive behaviour of materials, and the surface finish. One of the largest advantages of AJM is its ability to fabricate anisotropic patterns with low cost in comparison to conventional microfabrication methods, such as wet etching $[13,14]$. 


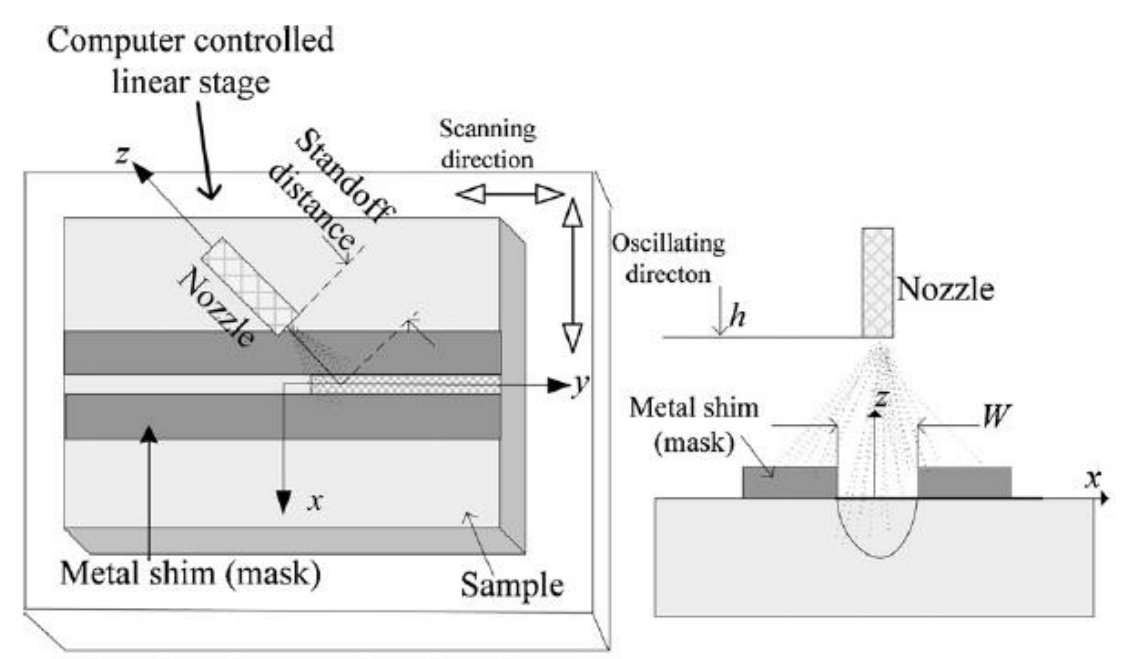

(a)

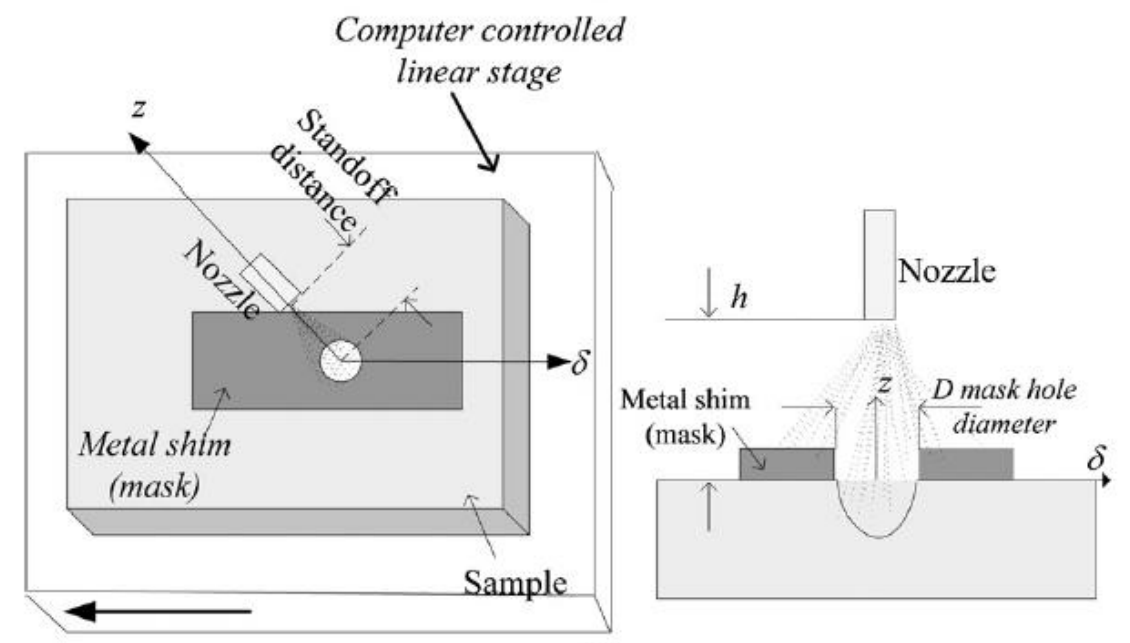

(b)

Figure 1.4 - Schematic of channel blasting apparatus [15].

The fabrication of micro features using AJM can be achieved either using masked or unmasked targets. In the AJM process of masked channels, the opening of the mask is smaller than the jet footprint on the surface, Fig. $1.4[13,16]$. The erosion across a central portion of the channel is relatively constant; however, this does not apply to the edges where there are interactions between the particles and the edge of the mask. This causes a reduction in the erosive power as more particles ricochet from the mask [17]. On the other hand, in unmasked micromachining using AJM, erosive power is known to be non-uniform. In addition, the jet footprint is fully exposed to the target surface, which can be used to machine larger planer areas [13]. 


\subsubsection{AJM vs. traditional micro-manufacturing methods}

Fabricating features smaller than one millimeter is known as micromachining, and it is used commonly in the production of, but not limited, (Microelectromechanical systems) MEMS, micro-sensors, and microfluidics devices. One of the main problems with traditional micromachining techniques such as chemical etching is the use of hazardous chemicals [18]. In addition, micro-milling using traditional cutting tools is time consuming due to the small size of the tooling during the process $[19,20]$. Micro-milling has other problems. Due to existence of submicronlevel dimensions, machined chip removal is difficult, and the very unpredictable cutting action causes sudden tool failure. Finally, since the milling is done on the micron scale, even a tiny defect on the tool or tool deflection can compromise the accuracy of the milling and finished product [19]. Zheng et al. [21] reported creating micro-features using electrical discharge machining (EDM). However, this technique is relatively expensive compared to other competing methods. Kucukturk and Can [22] listed some of the drawbacks of EDM as:

(1) Unnecessary tool wear due to EDM slow rate of material removal.

(2) High power required during the process.

(3) Cracking on the work piece may occur.

(4) Electrode wear causes difficulty in reproducing sharp corners and fillets on the work piece.

(5) EDM is highly useful in machining of conductive material but electrically non-conductive materials require an additional conductive electrode in order to be machined.

Ogura and Yoshida [23] are among the many who fabricated features such as micro-holes, using $\mathrm{CO}_{2}$ laser machining. The drawback of this method is the extreme temperature created by the laser that causes target surface thermal damage, and poor surface quality. In contrast to traditional methods, AJM has a directional etch capability that can produce microfluidic channels of non-uniform depth, using abrasive slurry jet machining [24], a similar approach to AJM. Ghobeity et al. [25] reported creating microfluidics channels using AJM using a sacrificial photoresist mask.

It is essential to mention the limitations of AJM. The resolution of unmasked AJM is limited since particles that diverge after exiting from the nozzle cause larger than anticipated channel geometries [26,27]. However, the use of masks compensates for particle divergence (Fig. 
1.4). In addition, the surface roughness of the features fabricated by AJM is relatively high. Therefore efforts have been made to minimize the surface roughness problem [28]. For example, Wensink et al. [28] improved the roughness of the channels after AJM by post hydrofluoric acid (HF) etching or high temperature annealing at $800{ }^{\circ} \mathrm{C}$. Haj Mohammad Jafar et al. [29] investigated the effect of AJM input parameters such as particle size, velocity, and incident angle on the unmasked channel roughness. Machined channels at lower impact angle had smoother surfaces. In general, the study showed that the roughness of channels was proportional to the kinetic energy; i.e. higher kinetic energy resulted in higher roughness due to larger chip removal from the substrate surface with each particle impact [29].

\subsubsection{Micro-mechanism of material removal}

Ghobeity et al. [15] stated that in general, solid particle erosion is divided into "ductile", "brittle" or both depending on the material properties distinguishing two different deformation mechanisms and machining conditions. The erosion mechanism of ductile systems involves cutting, extrusion, forging, and removal of target material at high strain rates; however, cracking and chipping are the dominant mechanisms of material removal in brittle materials [15]. The maximum erosion rate, i.e., the mass of target material removed per unit mass of incident erodent,

for brittle systems usually happens when the particles incident are perpendicular to the target surface. On the other hand, the erosion rate in ductile systems reaches maximum when the particles strike at a $15^{\circ}-30^{\circ}$ angle from the surface [30]. Figure 1.5 shows the relationship between the impact angle of eroding particle on the surface and erosion rate of both ductile and brittle materials. 


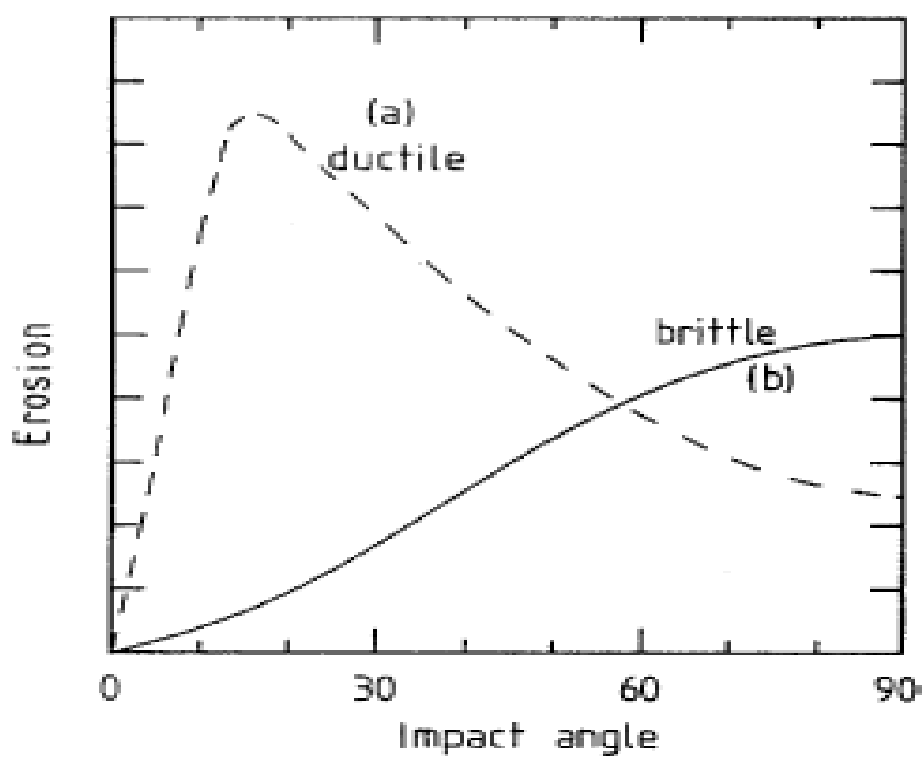

Figure 1.5 - Schematic of the variation of erosion with impact angle: (a) for ductile materials; (b) for a brittle materials [30].

Figure 1.6 is a schematic representation of some of the involved erosion processes. Microcutting as shown in Fig. 1.6a occurs by the impact of abrasive particles at small impact angles resulting in removing tiny chips. Formation of the lip occurs at the particle final track and then is later removed by other impacting particles. The parameters that affect the amount of volumetric material removed is a function of particles kinetic energy, angle of impact, and the target material's flow stress. Indentation occurs solely when the impact angles approach $90^{\circ}$. In addition, as it can be seen in Fig. 1.6b, ploughing deformation, as the material removal process, occurs if spherical particles are used. On the other hand, for brittle materials, the erosion mechanism, Fig. 1.6c, occurs by indentation fracture mechanics through the formation of micro cracks as well as crack propagation, and interaction, reported by Sheikh-Ahmad [31].

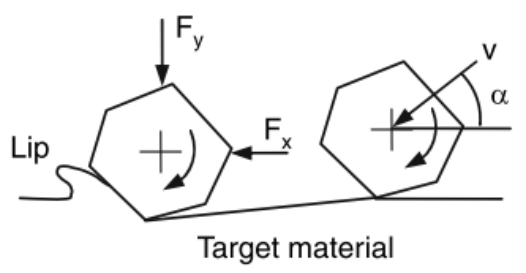

(a)

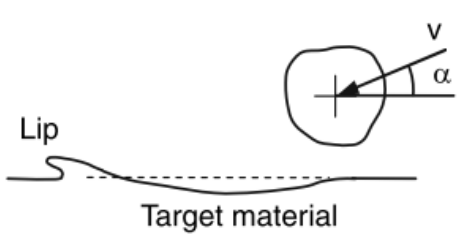

(b)

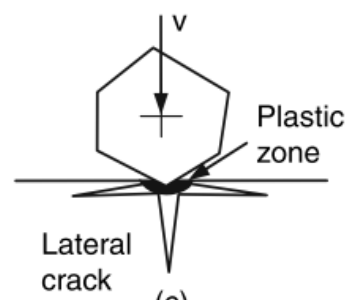

(c)

Figure 1.6 - Mechanisms of material removal by solid particle impact, (a) micro-cutting, (b) deformation, and (c) lateral cracking [31]. 
The use of various operating conditions such as angle of attack, erodent particle properties, and target characteristics results in different erosion mechanisms, ductile, brittle, or a combination of both. Several mechanisms have been widely studied and investigated for material surface removal by solid particle impact. One of the pioneering investigators of this field is Finnie [32], who studied mechanisms associated with cutting or displacing the target material. In another study by Tilly [33], it was concluded that the erosion process consists of two stages: i) the formation of raised lips and ii) the removal of those lips by a radial flow of fragments. This removal was caused by the impingement of particles impacting the surrounding area. Hutchings and Winter [34] reported that a lip can be formed from surface material sheared caused by the motion of a single spherical particle, which strikes the surface at a velocity that is higher than the critical oblique impact velocity. These lips in fact can be separated from the substrate through the material removal process.

As Hutchings reported [30] and Papini and Dhar [35] verified, the eroded surface quality and erosion rate are affected by not only the properties and structures of the erodent particles and target materials, but also the equipment input variables such as the velocity, size, shape, and impacting angle of the erodent particle. Hutchings [30] reported that the relative values of the hardness of the erodent and the target govern the operative material removal mechanism. The erosion rate resulting from small scale chipping mechanism are typically an order of magnitude lower than the lateral fracture mechanism, Hutching reported [30]. Using hard particles, two methods were used to attack the materials; namely, sliding over the surface (abrasive wear), and impacting freely resulting in erosive wear and both of course [30]. The local fracture phenomenon can be compared to the dimension of the particle; for instance, it was stated that the abrasive wear rates of a range of ceramics were increased by a factor of ten when wear occurred due to brittle fracture instead of plastic deformation.

One study conducted by Tilly and Sage [36] on solid particle erosion postulated that impacting particles initially cause pitting and extrusion of the target material. The report also hypothesized that the initial impact may results in particle fracture which may lead to "secondary damage" as the particle fragments move in radial directions relative to the point of initial impact. As a summary, they found the following: (i) In comparison with coarse erodents, small particles are less likely to fracture and break up while they require many impact cycles to erode chippings from the substrate. (ii) The erosion rate increases with increase in size of particles which also 
results in increasing fragmentation. (iii) Impact velocity has a significant effect on fracture and fragmentation of particles.

Materials can erode in different modes under different impact conditions. In an investigation done by Sheldon and Finnie [37], it was reported that aluminum experienced ductile erosion while soda-lime glass, sintered magnesium oxide, sintered alumina, and hardened tool steel all showed a brittle behaviour when eroded by large silicon carbide (CSi) particles at a speed of $153 \mathrm{~m} / \mathrm{s}$. The study showed that the erosion rate also depends on impact angle for ceramics and other nominally brittle materials. Nevertheless, it has also been shown that the brittle characteristic curve (Fig. 1.5, curve (b)) is not always evident for ceramic and other brittle materials. For example, Sheldon and Finnie [37] stated that by reducing the size of the silicon carbide erodent particles from $125 \mu \mathrm{m}$ to $9 \mu \mathrm{m}$, the maximum erosion angle of $90^{\circ}$ will reduce to a shallower angle. The findings were for tests conducted on brittle materials such as soda-lime glass, molded graphite, and magnesium oxide showing a brittle-to-ductile transition with change of particle size and not the temperature [37].

\subsubsection{Effect of process parameters on solid particle erosion}

There are various parameters that affect solid particle erosion. Misra and Finnie [38] have reported major parameters that have a direct influence on ductile materials. As some mentioned earlier, the parameters include 1) angle of impact; 2) particle rotation during the impact; 3) particle size; 4) particle velocity at impingement; 5) particle concentration in the fluid directed toward the substrate; 6) stress level on the surface of the substrate; 7) geometry and shape of the surface substrate; 8) surface properties; 9) abrasive particle shape and hardness; 10) carrier fluid; and 11) temperature of the surface and fluid. Barkoula et al. [39] divided these parameters into two categories of particle and target material properties. According to the review effective particle properties are:

- Particle size

- Velocity

- Roundness

- Hardness

- Kinetic energy

- Coefficient of restitution 
- Density

- Single mass

- Moment inertia

While the target material properties that can affect the AJM process are:

- Heat capacity

- Erosion Resistance

- Critical strain

- Fracture Toughness

-Enthalpy of melting

- Brittleness and ductility
- Grain diameter

- Hardness

- Grain molecular weight

- Melting temperature

- Density

- Depth of deformation
- Young's modulus

- Deformation energy

- Lame constant

- Incremental strain per impact

- Cutting energy

- Thermal conductivity

\subsubsection{Ductile erosion}

Ali et al. [40] suggest that the general oblique impact of a rigid particle on a flat surface, (Fig. 1.7 and Fig. 1.8), can divide into two categories: i) normal angle, indention-like, and ii) tangential, scratching-like. In the first case, the surface is pushed down by the particle and the material beneath is compressed. In addition, the particle tends to move sideways if this indentation continues; and then upward toward the free surface. The particle ricochets making a permanent crater on the surface and the formation of excess lips takes place, called "cantilever formations". Formation of this crater and surface displacement consumes the kinetic energy of the impacting particle $[40,41]$. 


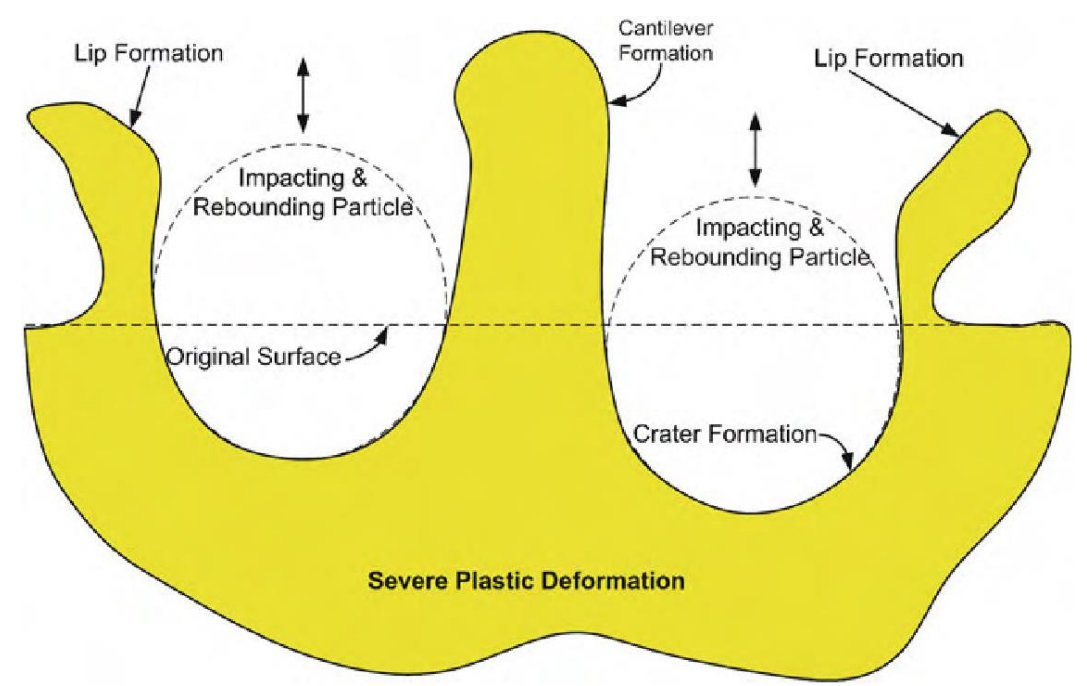

Figure 1.7 - Target material displacement and removal during continuous particles impact [41].

During the tangential motion seen in Figure 1.8 below, shear stresses dominate over compressive stresses in material removal. There is tensile stress on the trailing edge and more compressive stress on the leading edge of the particle, reported by Ali et al. [40]. The formation craters made during the tangential motion are due to sliding and friction effects.

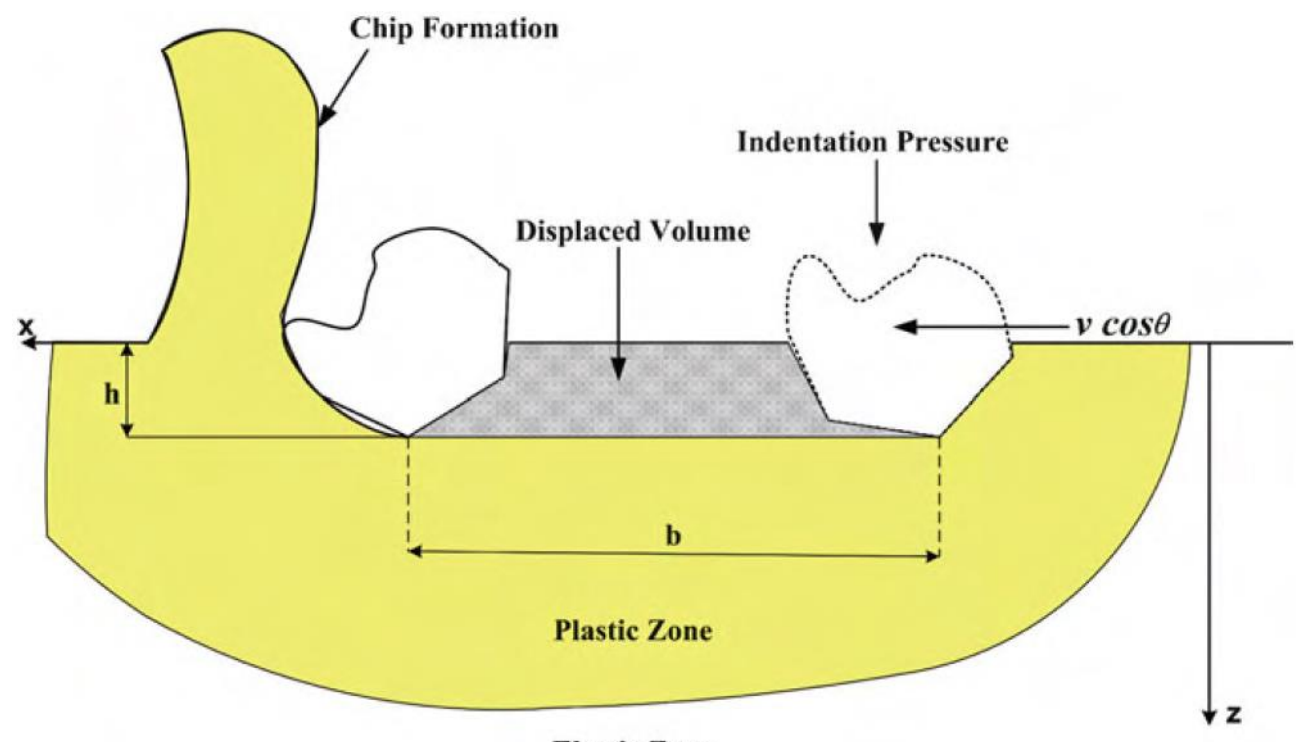

Elastic Zone

Figure 1.8 - Material removal mechanism when abrasive impact tangentially or scratching abrasive motion [40]. 
According to Ali et al. , the total material removal considering particle impact effects in normal mode and tangential mode can be expressed as [40,41]:

$$
\frac{V_{r}}{m_{p}}=k_{n} \frac{\left(v \sin \theta-v_{n}\right)^{2}}{H}+k_{t} \frac{\left(v \cos \theta-v_{t}\right)^{2}}{H}
$$

where $k_{n}, k_{t}$ are experimental coefficients, $v_{n}$ and $v_{t}$ are threshold velocities below which no material removal can occur, $H$ is the target material hardness, $m_{p}$ is the particle mass, $v$ is particle velocity, and $\theta$ is the angle of impact.

\subsubsection{Brittle erosion}

Brittle materials such as ceramics and glass, do not have considerable plastic deformation. Therefore, these materials respond to impact loads by fracture, forming cracks, and fragmentation of the affected region [40]. There are various types of cracks that can be formed in materials with brittle properties: i) ring cracks caused by elastic stress waves; ii) conical cracks affected by quasistatic tensile failure; and iii) radial, median, and lateral type cracks. The lateral cracks forms because of redistribution of stresses in the plastic zone at the crack tip during loading and unloading as shown in Fig. 1.9 [40].

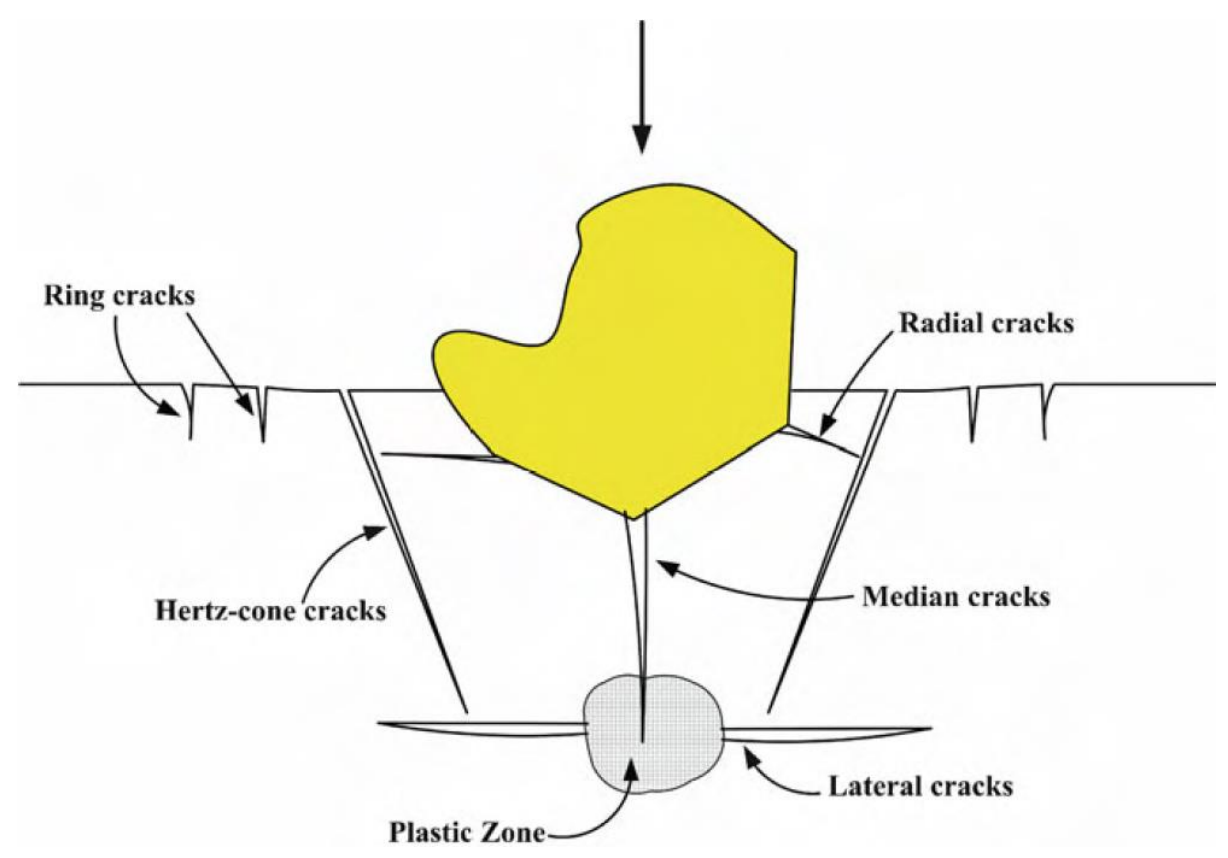


Figure 1.9 - Schematic of brittle erosion caused by cracking and fragmentation of surface material [40].

Aquaro and Fontani [42] reported that the fracture mechanisms depends on flaw sizes, the location, size, growth rate of the various cracks, and the interactions among various cracks. Generally, all brittle erosion models depend on the following terms [42]:

$$
V_{r} \propto \rho_{p}^{q_{1}} d^{q_{2}} \quad v^{q_{3}} \quad H^{q_{4}} \quad K^{q_{5}} \quad E^{q_{6}}
$$

where $\rho_{P}$ is particle density, $d$ is the particle size, $v$ is particle impact velocity, and $H, K, E$ are hardness, fracture toughness, and Young modulus of the target material, respectively. The exponents $q_{1}, q_{2}, q_{3}, q_{4}, q_{5}, q_{6}$ were found experimentally for each specific particle-target material grouping. For brittle materials such as ceramics, when a solid particle strikes their surface, lateral and radial cracks form. Most of the material removal is due to the lateral crack which propagates outwards and eventually reaches the surface to remove a chip, Fig. 1.10 [42].

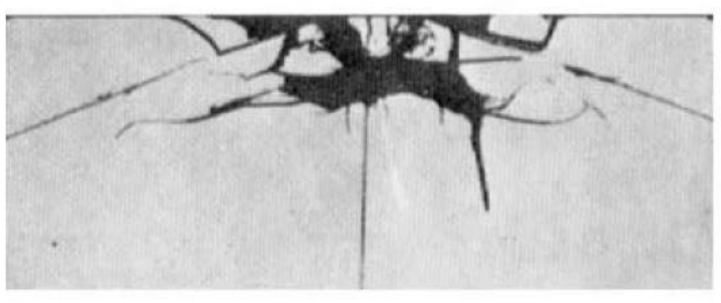

(a) Crater shape

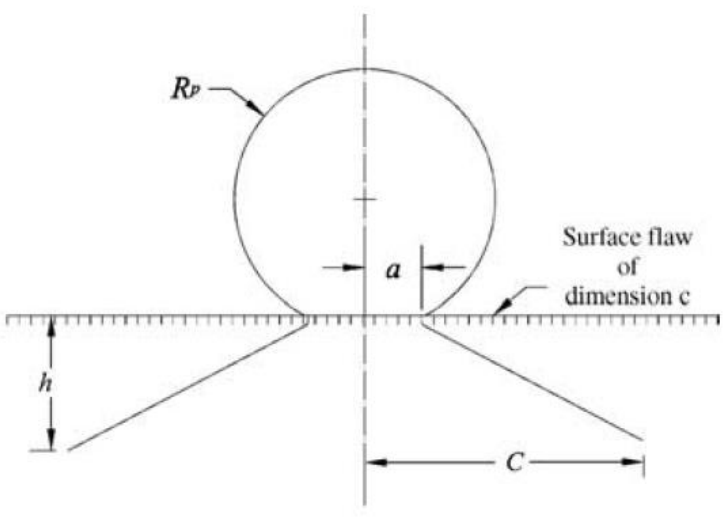

(b) Crack propagation

Figure 1.10 - Crater shape \& Crack propagation in brittle material [42].

The erosion rates in brittle systems are overestimated due to the oversimplification of the conditions under which lateral cracks cause chipping. Figure 1.11 shows a scanning electron microscopy (SEM) photograph of a typical lateral crack system. In this case, the test assumes that the lateral crack does not initiate at the bottom of the plastic zone, but outside the plastic zone. Using the same assumption, Verpui et al. [43] developed a simple simulation model of brittle material erosion process. It was reported that the roughness in the simulation model was much 
higher than the experimental surface roughness. With this finding, it was reported that there is a possibility that the lateral crack begins at the bottom of the indentation instead. Using the new assumption, the simulated model roughness improved, closer to the experimental results [43].

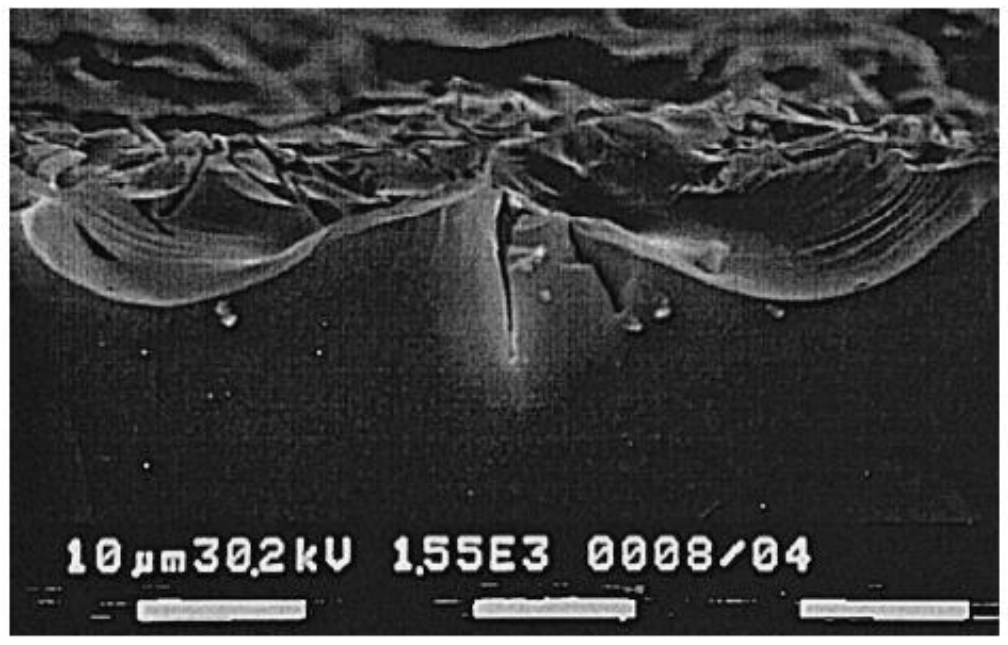

Figure 1.11 - SEM photograph of a lateral crack formation on a borosilicate glass target surface [43].

Haj Mohammad Jafar et al. [44] developed a numerical model that predicted the roughness as well as the erosion rate of a brittle material subjected to unmasked AJM process. The study concluded that by decreasing the angle of attack, the number of edge chipping increased; however, the major damage mechanism was caused by crater removal at both normal and shallow impact angles.

In a study by Basak et al. [45], it was stated that the formation of impressions that were described earlier are consistent with elastic-plastic material deformation. Looking at Fig. 1.12 below, the impressions can be explained in four different forms: (i) plastic indentation, with no cracks due to insufficient tensile stress (Fig. 1.12a) (ii) radial and lateral cracking with some added features due to crystallography (Fig. 1.12b) (iii) primarily lateral cracking with minor radial cracks (Fig. 1.12c) and (iv) radial and lateral cracking (Fig. 1.12d). 

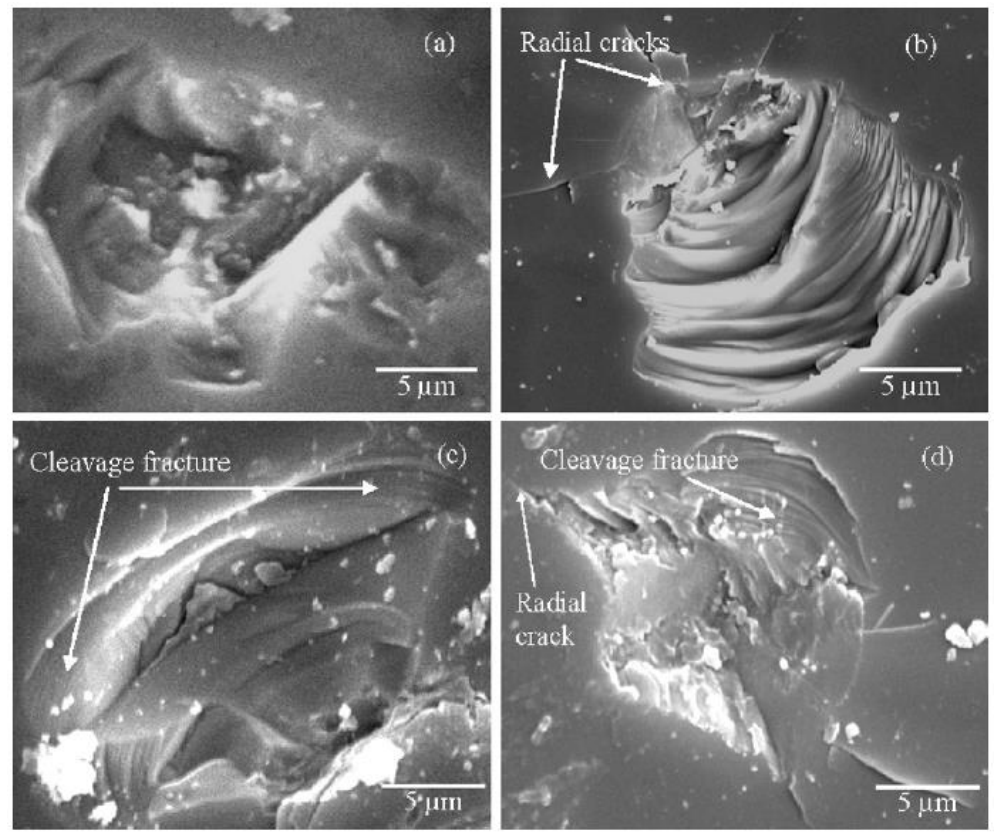

Figure 1.12 - Crater impressions are explained with elastic-plastic material theory [45].

Basak et al. [45] reported that all these types of impressions are formed from plastic indentation. However, they do differ in the extents of radial and lateral cracking (Fig. 1.12). The study also mentioned that the scratch type impression can also be explained according to the elastic-plastic framework. The detail of this impression is shown in Fig. 1.13 as following [45]:
(a) Scratch without any cracking
(b) Minor lateral cracking
(c) Some radial cracking
(d) Considerable lateral cracking 

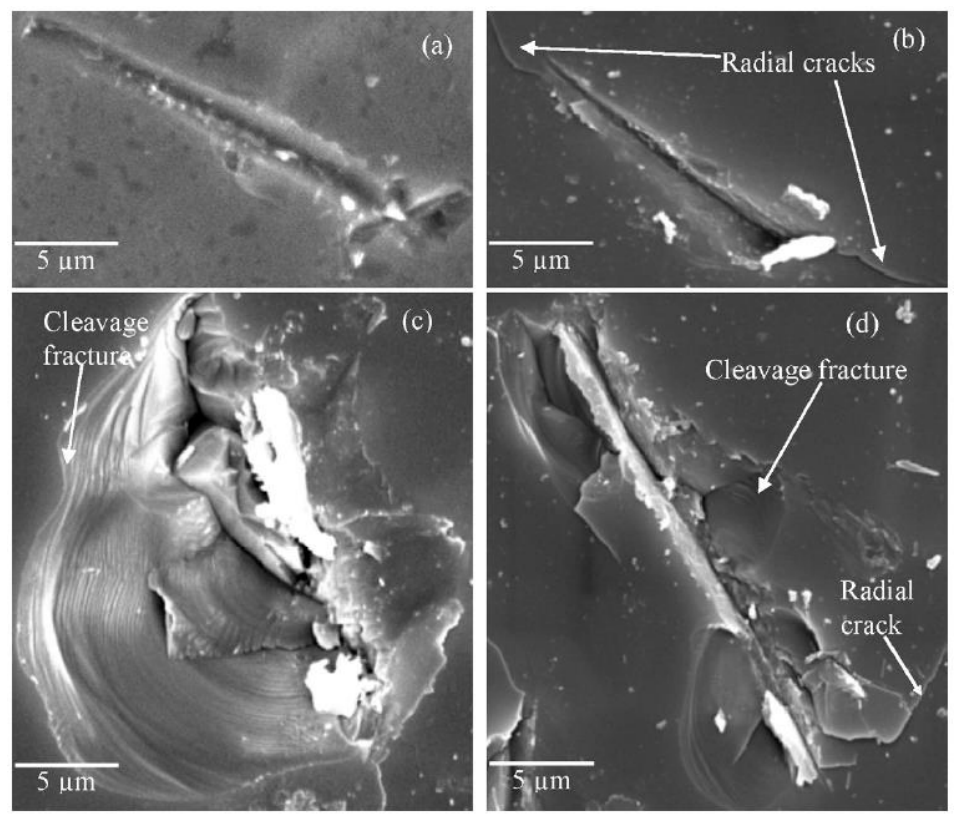

Figure 1.13 - scratch impressions are explained with elastic-plastic material theory [45].

It was observed that crack formation during elastic-plastic deformation was influenced by upward excursions. The cracks grow parallel to the surface of the substrate and shift in this direction most likely because of the possibly low surface energy crystallographic planes, illustrated as steps in the cleavage fracture zone, Fig. 1.14. In addition, Basak et al. [45] also reported microindents are produced by debris created or formed from impact fracture. However, they emphasize the removal mechanism follows elastic-plastic models when the impact angle is normal to the target surface, where compressive and tensile forces have significant roles, while at $30^{\circ}$ and $45^{\circ}$, shear stresses act as well as compressive and tensile stresses, which may affect the mechanism of material removal. 


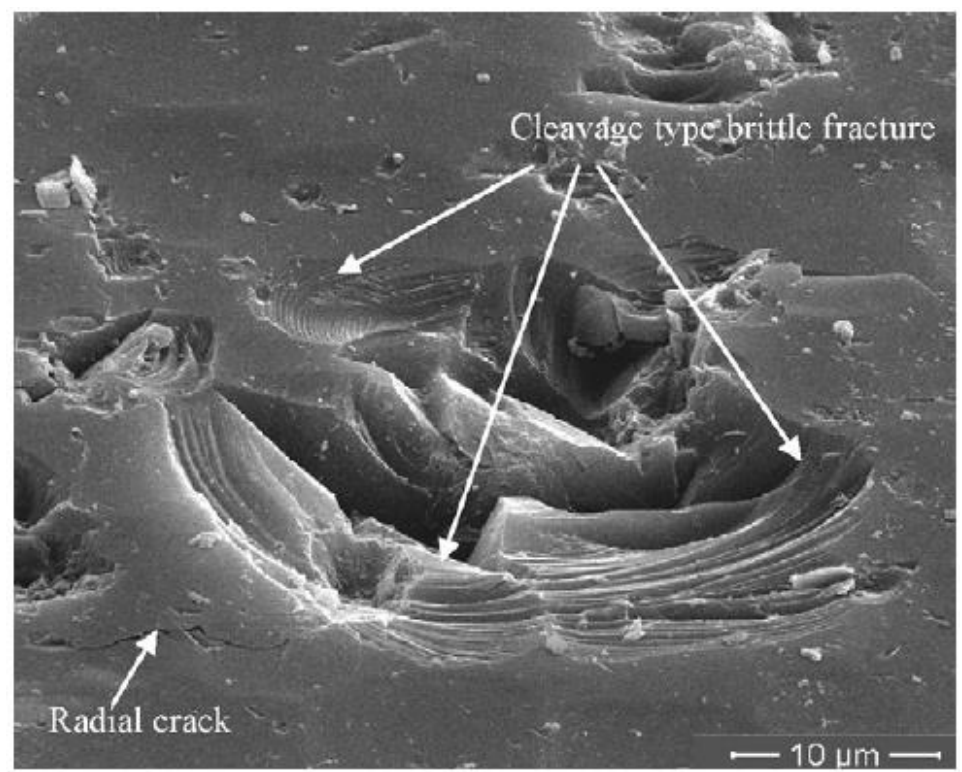

Figure 1.14 - Schematic of a crater impression showing cleavage fracture on a brittle material [45].

In 1996, Zeng and Kim [46] concluded that the total energy induced on the target when a particle strikes consists of plastic deformation energy, stress wave energy, and residual strain energy. They hypothesized that the plastic deformation energy converts to both heat and potential energy. The converted potential energy manifests as residual strain. Fractures and internal friction within the target material are caused by the stress caused by particle incidents. Finally, the residual strain occurs by either particle fragmentation or rebounding [46]. Figure 1.15 presents a cutting action that removes the top layer of material through plastic flow. Fractures occurs along the grain boundaries caused by the stress wave.

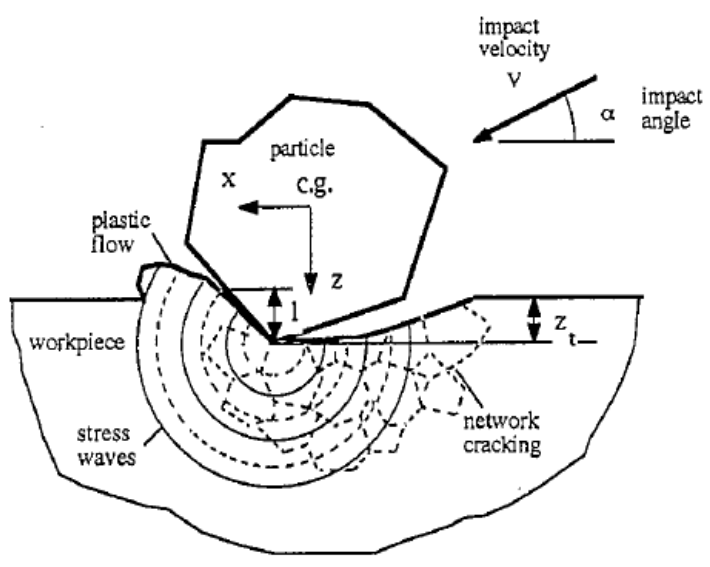

Figure 1.15 - Schematic of two material removal modes: (1) scratching at the contact area (2) network cracking at its neighborhood [46]. 


\subsubsection{Microfluidic device fabrication using AJM}

Solignac et al. [47] used AJM and a metal mask to fabricate microfluidic channels having $100 \mu \mathrm{m}$ wide and $30 \mu \mathrm{m}$ depth, to form a capillary electrophoresis chip , Fig. 1.16. In this study, a thin metal sheet was used as a sacrificial mask with high erosion wear resistance that was placed and clamped on the substrate.

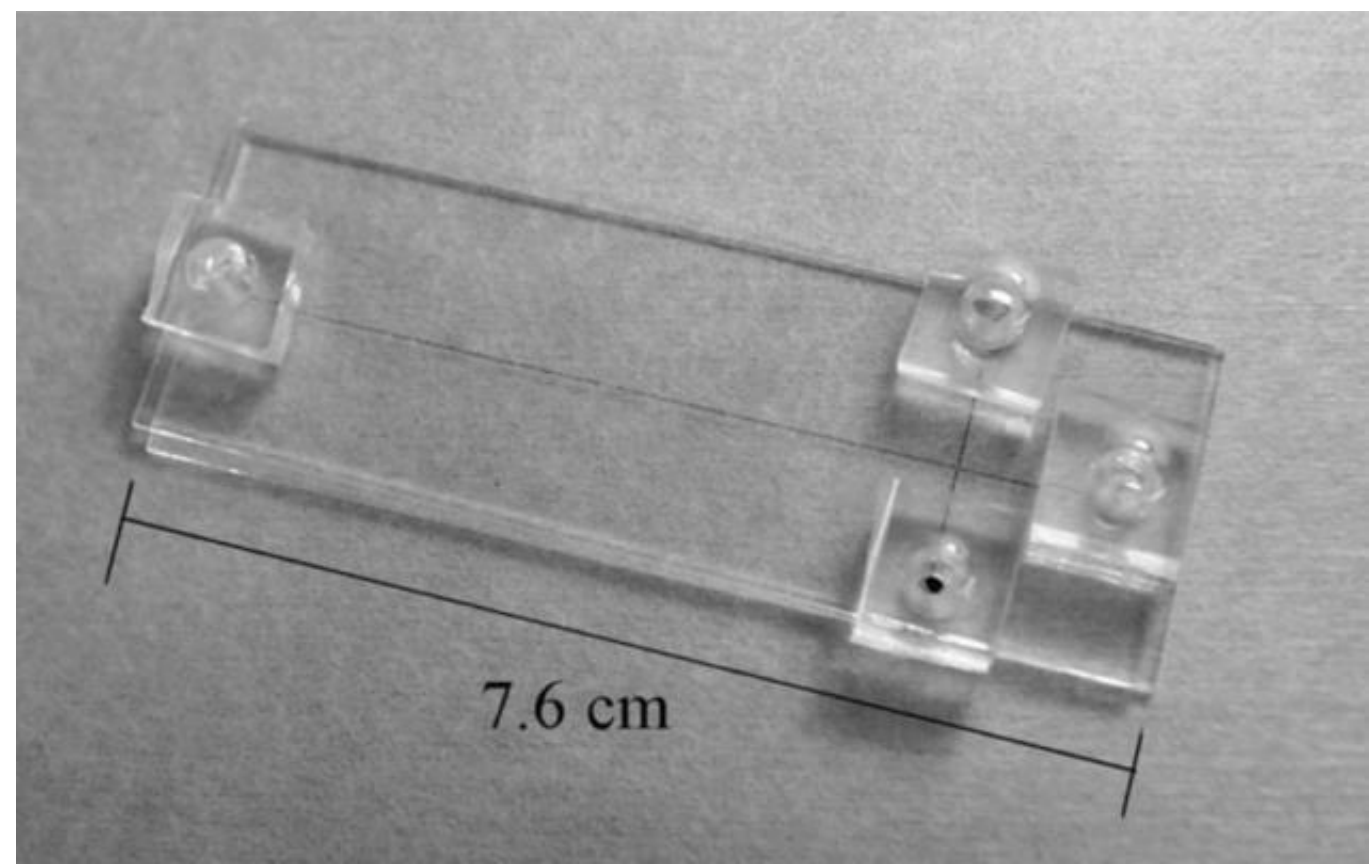

Figure 1.16 - Capillary electrophoresis chip fabricated by AJM with PDMS inlets/outlets glued [47].

Burzynski and Papini [48] used a High Tack RapidMask (RM) (IKONICS Imaging, Duluth, MN, U.S.A.) in their fabrication of various traditional and inclined microfluidic channels. The RapidMask is a self-adhesive photoresist that is ultraviolet light sensitive. The uncured RM erodes with ductile properties when blasted with particles during AJM. Burzynski and Papini found that the maximum erosion on the uncured regions occurred when particles strike the surface at relatively shallow angles $\left(<40^{\circ}\right)$. On the other hand, the cured regions of RM have brittle erosive behaviour, i.e. erosion is maximum when particles strike normal to the target surface, Sec 1.1.4. The photomask used to pattern the RM was made using CAD software and printed using a highresolution printer. The photomask was placed on the RM and exposed to a fused quartz silicon dioxide curing lamp $(5.04 \mathrm{~W} / \mathrm{mm})$. After the desired pattern was cured on the RM, it was carefully 
placed on the substrate to be eroded by the AJM. As mentioned above, the UV cured regions of the RM (desired pattern) became brittle. Maximum ductile and brittle erosion occur when the impact angle of particles are $<30^{\circ}$ and $90^{\circ}$, respectively (Section 1.1.4). Therefore, when the RM on a substrate was blasted by the abrasive particle while the nozzle was normal to the surface of the target material, the brittle (UV exposed) portion of the RM eroded faster. Therefore, the particles reached the substrate under brittle section resulting in etching of the desired pattern on the target. Figure 1.17 is a schematic of the placement of the RM and substrate.

Ghobeity et al. [25] also used a high tack self-adhesive RapidMask® (RM) to create microfluidic chips by AJM. The study reported fabricated feature sizes from $50-300 \mu \mathrm{m}$ using the technique. The fabricated channels are considered as a 2D microchannel since there is uniform depth along the length of the channel.

A more detailed review of microfluidic chip fabrication using AJM and Rapidmask can be found in Section 2.1.
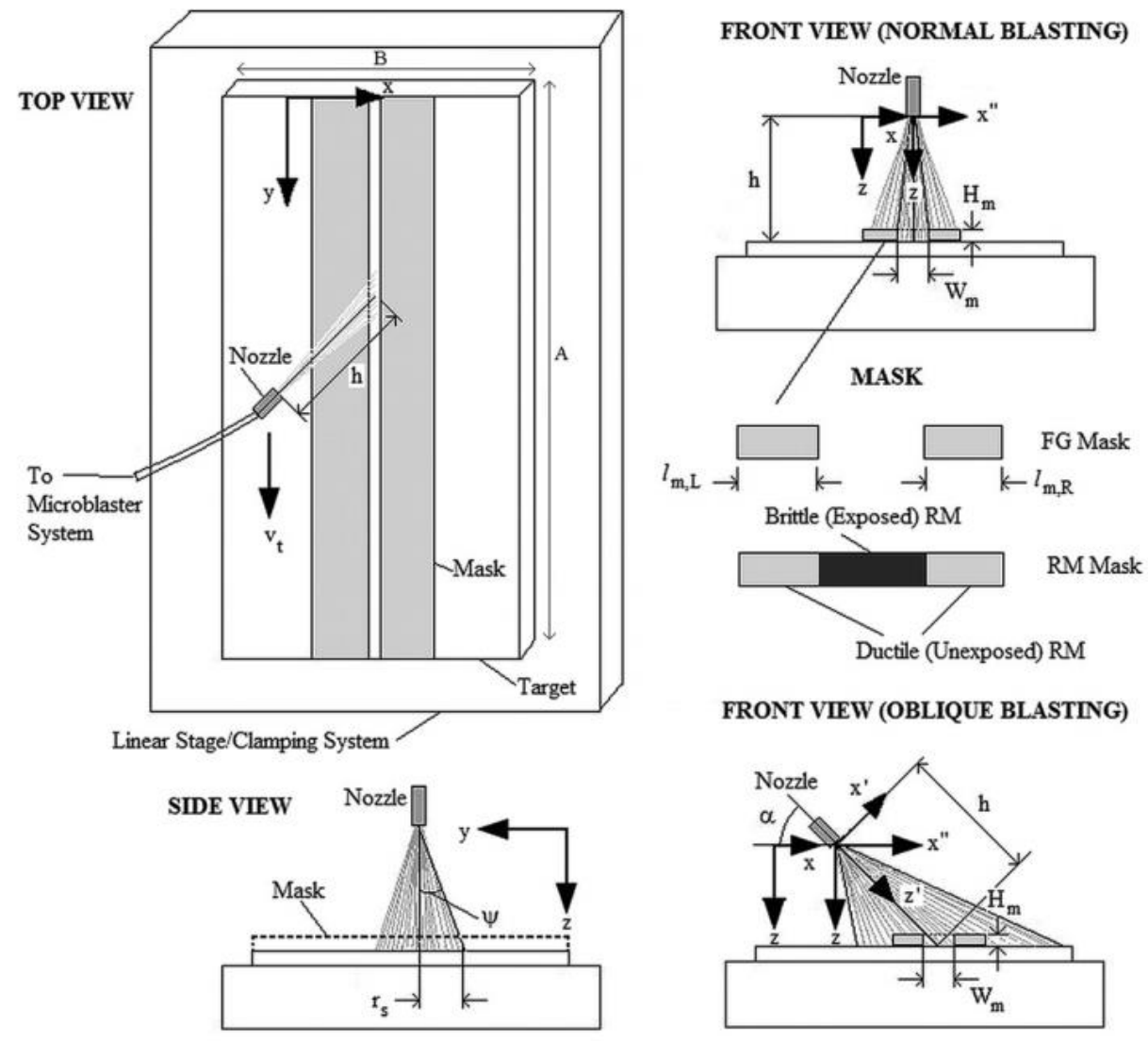

Figure 1.17 - Schematic of AJM apparatus to fabricate micro-channels using sacrificial masks [48]. 


\subsection{Summary}

In previous sections, a number of micromachining techniques, erosion mechanisms, and fabrication of microfluidics chips were discussed. In this section, some of the limitations and issues surrounding microfluidics devices fabricated by AJM using RapidMask are discussed.

As explained in Section 1.1.9, there have been number of studies on fabricating microfluidics chips using masked AJM [25,47]. Machining channels using masked AJM allows control on channel width, with a smaller achievable width. Using metal masks, however, may compromise the channel width due to the mask undercutting that occurs during AJM. Undercutting is caused as particles escaping between small gaps between the mask and substrate surface [13]. In studies mentioned in Section 1.1.9, RapidMask was used as an alternative material for masked AJM. Due to its self-adhesive nature, small thickness, and ultraviolet exposure behavior, it was found to be suitable for fabricating microchannels on glass with microfluidic applications [25,48]. As mentioned in Sec 1.1.1, fluid flow focusing can improve the analysis of cell, beads, and other biological samples [1-6]. Three dimensional flow focusing microfluidic devices fabricated using PDMS and soft lithography techniques have been reported [1]. However, AJM and RapidMask have never been implemented together to fabricate three-dimensional flow focusing devices.

Though RapidMask has been used in AJM applications, there is not enough information for its use in micro-scale applications. RapidMask has been used for larger scale industrial sand carving artistry, where the accuracy and mask blast resistance is not as important as it is in AJM application where channels with specific geometries and aspect ratio are desired. Furthermore, its application to exploit one of AJM's major advantages, i.e. its ability to gradient etch threedimensional features, has not been yet explored.

\subsection{Objectives}

The overall objective of the thesis was to determine whether RapidMask could be effectively used with AJM to machine three-dimensional microfluidics devices. The specific objectives are:

(i) Determine a quantifiable parameter that could be used as a measure to characterize the ultraviolet light curing cycle of RapidMask. This will be shown in Chapter 2 to be the UV light energy density. 
(ii) Determine the effects of curing unit configuration, UV energy density, and sample thickness, position, and orientation, on the accuracy of the pattern transfer, and ultimately the geometry of resulting machined micro-features.

(iii) Develop a novel method to stack multiple masks with higher erosion resistance in order to fabricate aspect ratio channels.

(iv) Fabricate a three-dimensional flow-focusing microfluidic chip using RapidMask and AJM and experimentally show the chip's potential in microfluidic and biomedical applications. 


\section{Effect of curing parameters and configuration on the efficacy of ultraviolet light curing self-adhesive masks used for abrasive jet micro-machining}

This chapter focuses on the curing parameters and configurations of RapidMask (RM) used for AJM to achieve the most accurate micro-channels on borosilicate glass.

This chapter is based on the following submitted paper (currently under review):

F. Ahmadzadeh, S. S. H. Tsai, and M. Papini, (2016), Effect of curing parameters and configuration on the efficacy of ultraviolet light curing self-adhesive masks used for abrasive jet micro-machining. Review for Precision Engineering

\subsection{Introduction}

Abrasive jet micro-machining (AJM) uses a jet of particles, directed through a patterned erosion-resistant mask, to etch features for the micro-fabrication of a wide variety of devices. For instance, Schlautmann et al. [49] reported fabricating electrophoresis capillaries, Belloy et al. [50] fabricated glass inertial sensors, Park et al. [51] etched micro-molds in steel, Yamahata et al. [52] machined ball valve micro pumps in glass, and Ghobeity et al. [25] generated microfluidic chips in glass.

There have been a number of studies related to micro-device fabrication using AJM with patterned masks made from various metallic and polymeric materials. For example, Wensink et al. [53] examined two polymers that could be structured lithographically on glass, and studied the use of electroplated copper as a mask attached to a glass substrate over a titanium binding film. The study showed that the resulting copper mask had high erosion resistance, and channels as small as $50 \mu \mathrm{m}$ in width could be fabricated in glass. Liao and Chen [54] fabricated 240-575 $\mu \mathrm{m}$ microgrooves and holes on brittle silicon wafers using AJM with lithography. First, the substrate was preheated at $80^{\circ} \mathrm{C}$ and coated with a water-soluble resin, a mixture of polyvinyl alcohol and polyvinyl acetate. After drying the first layer, they formed a second layer using a combination of urethane acrylate oligomer, photopolymerization, and an initiator with strong adhesion to the first layer and strong resistance to AJM. After the layers were formed, photo-etching was used to achieve desired patterns which were then machined using AJM. Pawlowski et al. [55] used epoxy 
and polydimethylsiloxane (PDMS) masks attached to glass substrates, in conjunction with lithography techniques, to achieve feature sizes of 20 - $30 \mu \mathrm{m}$ after blasting the glass with $10 \mu \mathrm{m}$ diameter aluminum oxide particles. Lomas et al. [56] used PDMS and SU-8 as masks on stainless steel to make a precision hot embossing metal mold. Park et al. [57] reported using AJM to fabricate microgrooves as small as $80 \mu \mathrm{m}$ on glass using an ultraviolet (UV) light hardening polyurethane mask, BF405. Preparation of the masks, however, required a complex a multi-step process. Belloy et al. [14] reported creating complex microstructures in glass with aspect ratios between 5 to 10, by using steel masks attached to the glass, and rotating the specimen while machining at both normal and oblique incidence. Yagyu et al. [58] presented a rapid prototyping method to fabricate a glass chip using AJM and direct laser patterning of a polymer, AuPU4, as a mask coating on a glass substrate.

A number of authors have compared the performance of different mask materials in AJM applications. For example, Slikkerveer et al. [59] investigated the use of three different elastomeric coatings as AJM mask materials, a $100 \mu \mathrm{m}$ laminate dry-film resist (Ordyl BF410), a flexographic printing material (LF55G1), and ab aliphatic urethane diacrylate oligomer film (Ebecryl 270), and found that the maximum erosion occurred at oblique angles in all three cases. All three materials were polyurethane (PU) acrylate based, suitable for photolithography, and it was found that the LF55G1 was the most erosion resistant. Achtsnick et al. [60] studied the erosion resistance and pattern transfer accuracy of a variety of metal, elastomer and photoresist AJM mask materials. They reported that metal masks were generally more suitable for high particle velocities and fast production processes, but that the minimum achievable feature width was limited to $300 \mu \mathrm{m}$. For electroplated copper masks, the achievable mask thickness was limited to 10-30 $\mu \mathrm{m}$. Electroformed zinc was found to be a good alternative because it could be applied in thicknesses of $1 \mathrm{~mm}$ or higher, resulting in a large erosion resistance, albeit at the expense of a larger minimum achievable feature size. They also studied four types of self-adhesive elastomer foils and concluded that they were suitable for more complex pattern transfers, but did not perform well under higher air pressures due to their elastic behavior. They found that the epoxy-based photoresist SU-8 was a good compromise to use in order achieve improved accuracy, although the equipment to prepare the mask was relatively expensive.

Self-adhesive masks are of particular interest in AJM applications because, in contrast to the multi-step procedures described above that involve masks applied as coatings, self-adhesive 
masks can be patterned very easily, dry-processed, and easily adhered to the target. The technique used for self-adhesive masks is straightforward if a proper halftone image and a suitable ultraviolet (UV) light exposing unit are used. As shown in Figure 2.1, the desired pattern is first printed on a clear acetate transparency sheet. The sheet is then laid over the unexposed mask material and subjected to a dose of UV radiation. The areas below the black portions of the acetate sheet are not exposed to UV light, and remain erosion resistant, while portions below the clear areas cure, becoming brittle and thus easily eroded. The self-adhesive mask is then attached to the target substrate and blasted using AJM to fabricate the desired patterns shown in Figure 2.2. For example, Ghobeity et al. [25] used a high tack self-adhesive RapidMask® (RM) (IKONICS Imaging, Duluth, MN, U.S.A.) to create microfluidic chips, and achieved feature sizes from $50-300 \mu \mathrm{m}$. Burzynski and Papini [48] compared the performance of two types of RM with steel contact masks on a glass substrate, and reported that, because of its higher erosion rate, the aspect ratio, defined as the channel depth divided by its width, was limited to 1 using RM, as opposed as 3 using the steel. They used the measured erosion rate to construct a level-set based surface evolution model that could predict the shape of both the eroded channel cross sections, and also the eroded mask.

Although self-adhesive UV curing masks such as RM have been used in AJM applications, there is a lack of information regarding the optimum curing conditions. Such masks were originally developed for the much larger-scale application of sand-carving artistic patterns, where the demands regarding the pattern transfer accuracy and mask durability are not as stringent as they are for AJM applications where higher aspect ratio and smaller features are required. Therefore, the manufacturer-provided curing instructions provide only basic guidelines such as the recommended time to cure for a particular curing unit, reported in RapidMask user guides [61,62]. In this chapter more fundamental data will be presented for RM materials regarding the effects of curing unit configuration, UV energy density, and sample thickness, position and orientation, on the accuracy of the pattern transfer, and ultimately the geometry of resulting machined microfeatures. 


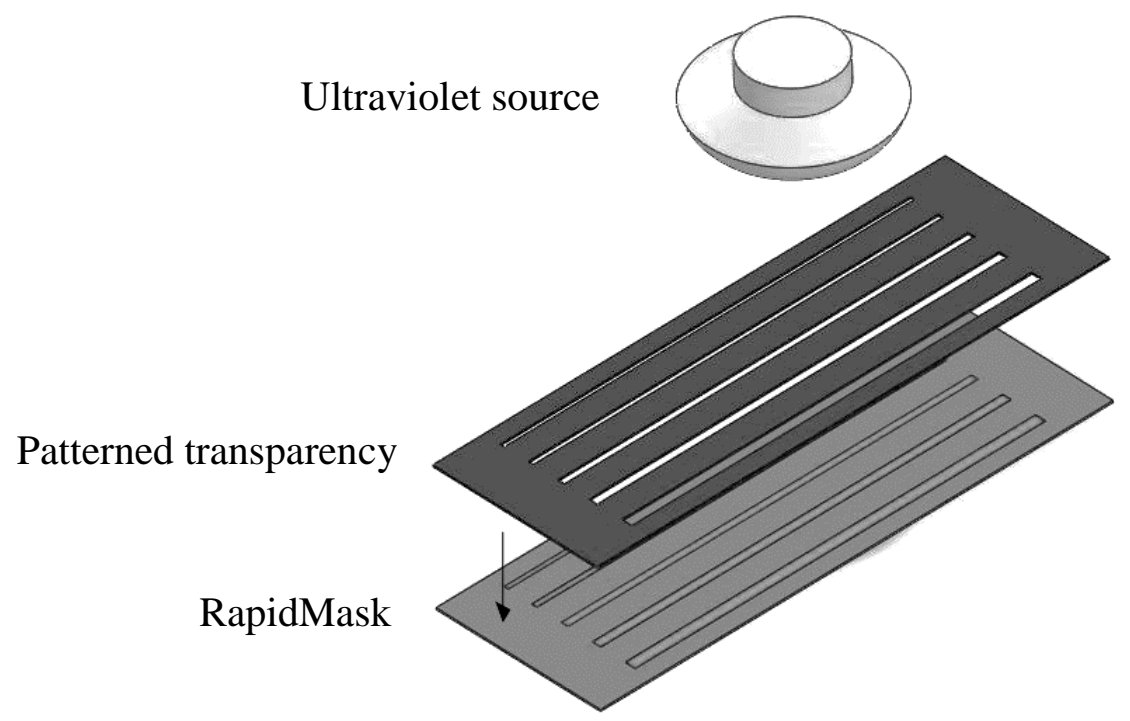

Figure 2.1 - Procedure to cure the RM using a patterned transparency that allows UV light from the source to pass through the transparent patterns of the photomask. The UV light is transferred onto the photosensitive RM film.

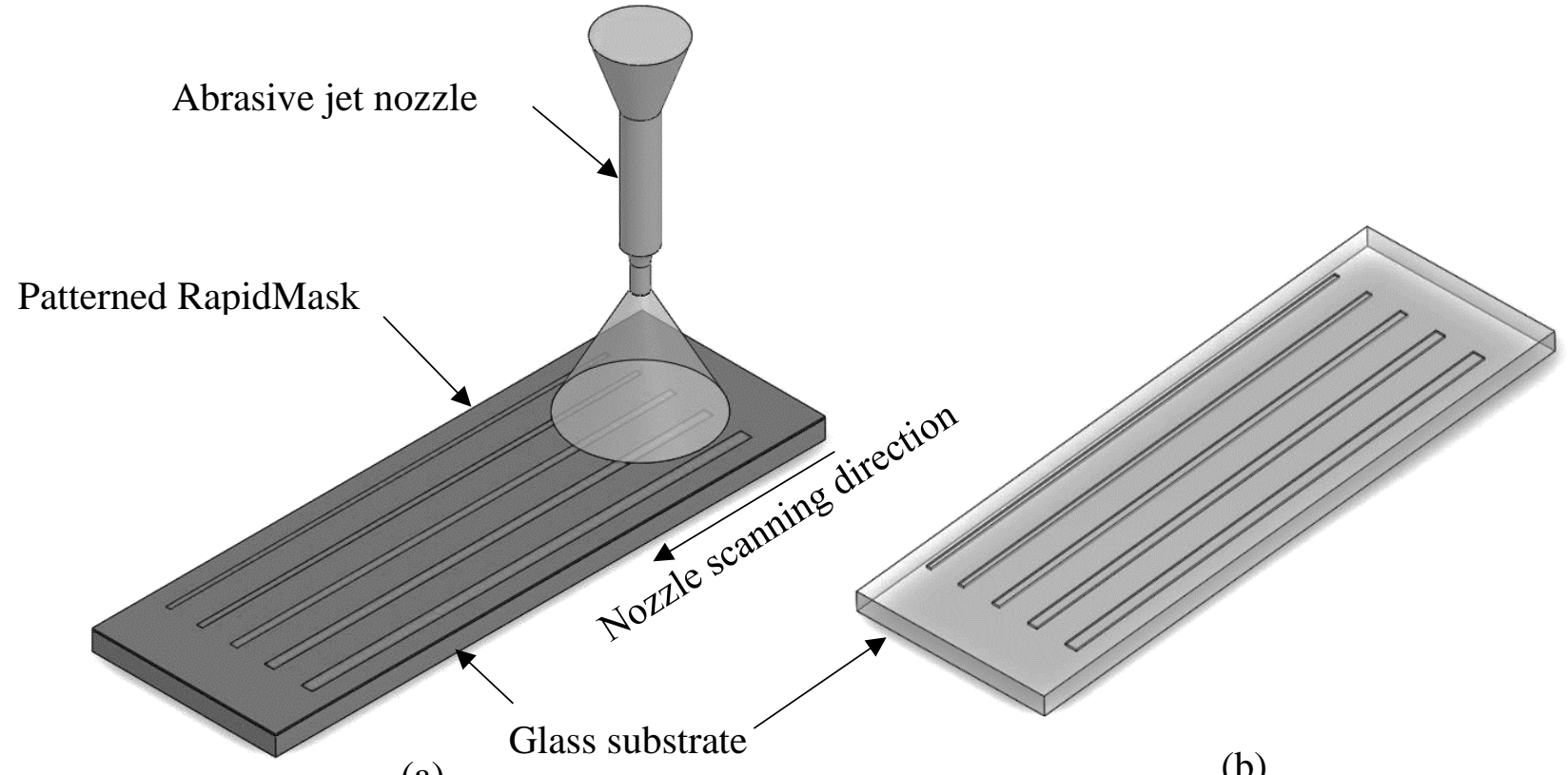

(a)

(b)

Figure 2.2 - Procedure to etch the surface of the glass. The patterned RM is attached to the glass, and AJM is used to transfer the pattern to the glass substrate. 


\subsection{Experiments}

The performance of the RM film under various cure conditions was evaluated in terms of the accuracy of the pattern transferred both onto the film (i.e., width of the cured features on film before AJM compared to the width of the lines on the photomask), and that of the features actually eroded into the borofloat glass target. There were obvious color and texture differences (Fig. 2.3), visible to the naked eye, between the uncured and cured areas of the mask, making measurement of the cured pattern transfer accuracy possible using an optical microscope and image analysis (Clemex intelligent microscopy, Les Technologies Clemex Incorporated, Longueuil, Quebec, Canada). The masks were then attached to the glass targets and subjected to AJM (Section 2.2.2), thus eroding away the brittle cured RM (Fig. 2.3) and exposing the glass to the abrasive jet. The topographies of the eroded features were then determined using an optical profilometer (NANOVEA ST400 Micro Photonics Inc., Irvine, CA, U.S.A.) with a depth and lateral resolution of $25 \mathrm{~nm}$ and $0.1 \mu \mathrm{m}$, respectively. All pattern transfer assessments were repeated four times, resulting in a standard deviation of $\sim 3-7 \mu \mathrm{m}$.

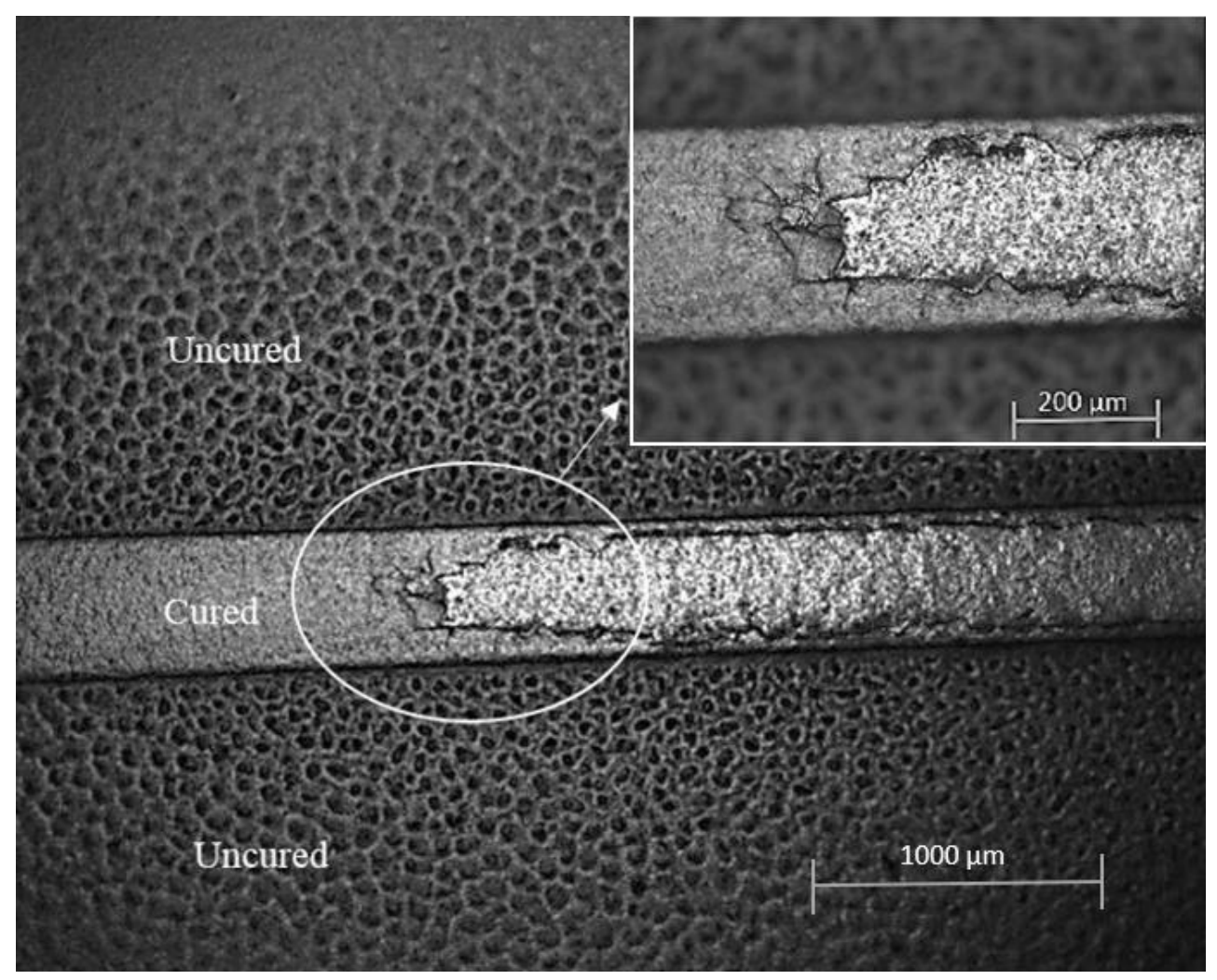

Figure 2.3 - Microscopic images of cured and uncured regions of RM after exposure to the UV unit and being eroded by AJM up to the circled region. 


\subsubsection{Patterns tested}

All the tests were conducted using High Tack (HT, $100 \mu \mathrm{m}$ thick), and High Detail (HD, $50 \mu \mathrm{m}$ thick) RapidMask® (RM) photoresist films (IKONICS Imaging, Duluth, MN, U.S.A.). The RM film was a mixture of Acrylic acid, triester with 2-ethyl-2-(hydroxymethyl)-1,3-propanediol, Ethanone, 2, 2-dimethoxy-1, and 2-diphenyl [63] with density of $1292 \mathrm{~kg} / \mathrm{m}^{3}$ [48]. Five patterns of straight $25 \mathrm{~mm}$ long channels with widths of 250, 300, 400, 500, and $600 \mu \mathrm{m}$ were designed using computer-aided design (CAD) software (AutoCAD 2016, Autodesk, Inc., Dan Rafael, CA, U.S.A.) and printed onto a transparency sheet (25,400 dpi, CAD/ART Services Inc., Bandon, OR, U.S.A.) to be used as photomasks. The designs are representative of typical channel widths used in relatively large microfluidic devices. Each photomask was attached to the RM using paper tape, and was exposed to UV light, to transfer the pattern on to the film, as shown in Figure 2.1. Figure 2.4 shows an example of a patterned transparency photomask (Fig. 2.4(a)), and the pattern transferred to an HT mask (Fig. 2.4(b)).

(b)

(a)
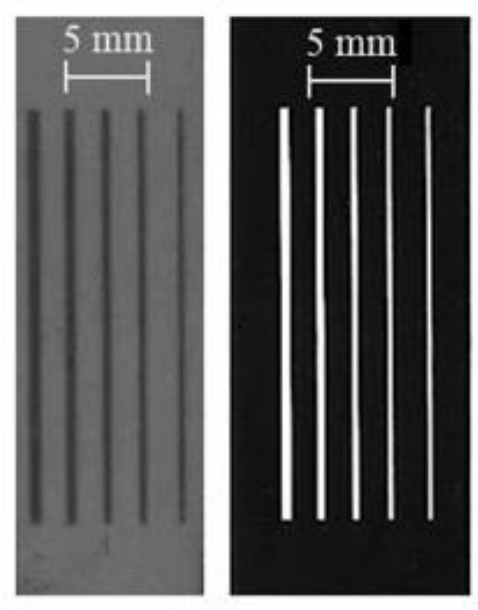

Figure 2.4 - Image of (a) negative photomask with pattern design used and (b) exposed patterned RM with distance of $\sim 1.5-2 \mathrm{~mm}$ between the channel patterns.

\subsubsection{AJM blasting experiments}

The AJM experiments were performed using an AccuFlo AF10 Micro-Abrasive Blaster, (Comco, Inc. Burbank, CA, U.S.A.) set at a $200 \mathrm{kPa}$ air pressure. To minimize the moisture in the room and the powder reservoir, a combination of a refrigeration air dryer, a dehumidifier, and a 
desiccant filter were used at the air inlet. As reported by Ghobeity et al. [64], an electric mixer with variable speed control (Arrow Model 850, Arrow Engineering Co., Inc., Hillside, NJ, U.S.A.) was used in the powder reservoir, which was kept at a constant powder level to achieve a relatively uniform particle mass flow rate, Fig. 2.5.

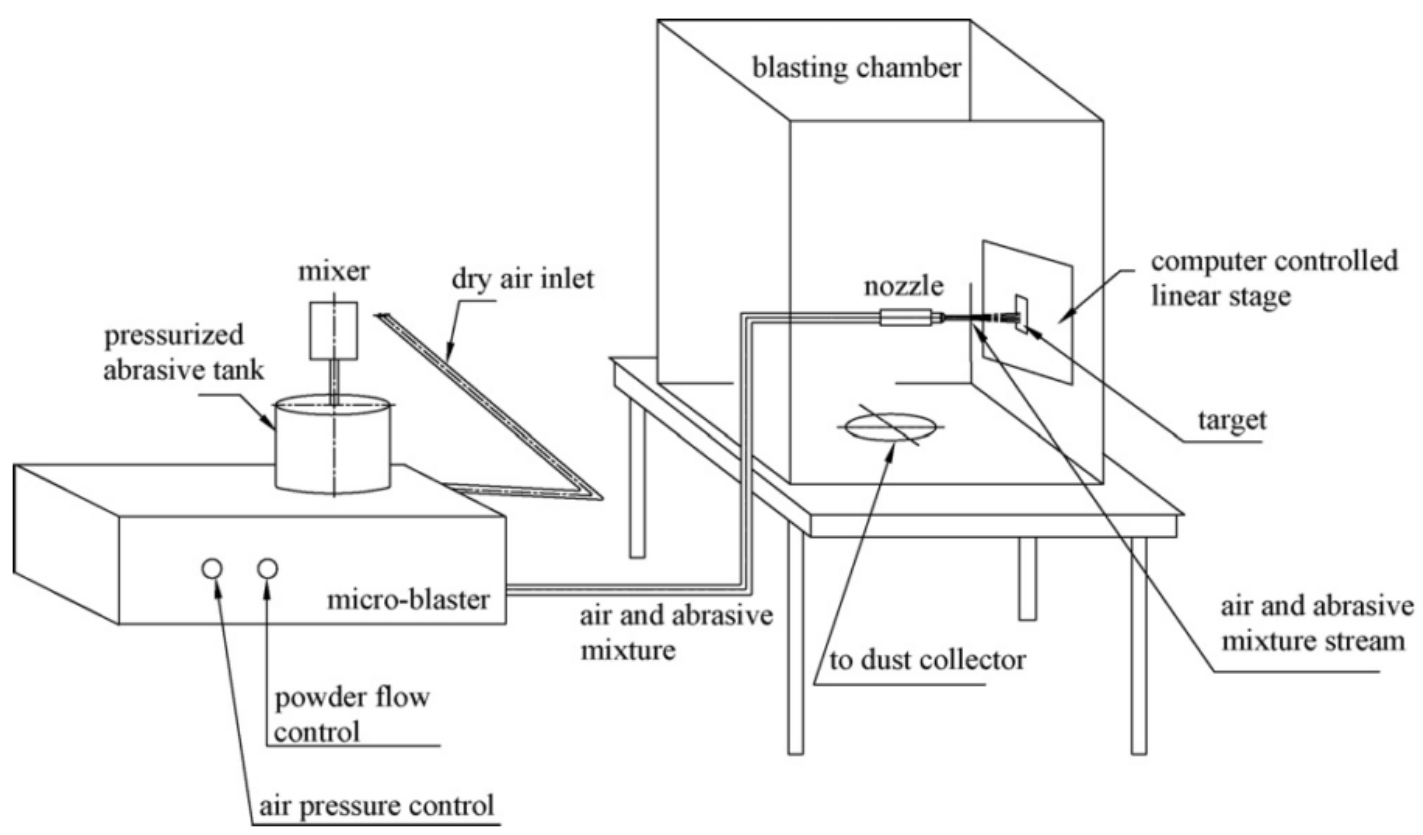

Fig. 2. Experimental apparatus.

Figure 2.5 - Schematic of abrasive jet micromachining experimental apparatus [65].

Aluminum oxide $\left(\mathrm{Al}_{2} \mathrm{O}_{3}\right)$ powder (Comco Inc. Burbank, CA, U.S.A.) with nominal $25 \mu \mathrm{m}$ particle diameter was used in all the experiments. The particle size distribution was found to be log-normal, as reported in [64]. After curing the RM under UV light with desired patterns, samples were adhered on $3.3 \mathrm{~mm}$ thick borosilicate glass targets (Borofloat, Swift glass Co. Inc., Elmira, NY, U.S.A.). The glass samples with RM on top were then clamped on a programmable computercontrolled linear stage (Aerotech Inc., Pittsburgh, PA, U.S.A.). The stage could be controlled in two directions independently with positioning resolution of $5 \mu \mathrm{m}$ that allowed constant linear motion under the fixed jet. A relatively large $0.3 \times 3.8 \mathrm{~mm}$ rectangular nozzle (Comco Inc. Burbank, CA, U.S.A.) was used for all experiments with jet scanning direction parallel to the channels pattern on the RM (Fig. 2.2). The nozzle was kept at a constant standoff distance of 20 $\mathrm{mm}$ from the surface for all tests, yielding an average particle velocity of approximately $150 \mathrm{~m} / \mathrm{s}$, 
estimated from the velocity model of Li et al. [66], assuming a particle diameter of $25 \mu \mathrm{m}$. The mass flow rate of the abrasive jet was measured before and after each experiment. This was done by collecting abrasive particles during $2 \mathrm{~min}$. with the nozzle blasting into a cylinder with an air filter sealed on the top. All of the experiments were performed at particle mass flow rates between 7.5 and $9 \mathrm{~g} / \mathrm{min}$, unless otherwise reported. The change in the mass flow rate during the $3 \mathrm{~min}$. experiments was always less than $12 \%$.

\subsubsection{Effect of UV curing unit geometry and energy dose}

Two different commercial UV curing units were tested: a cylindrical curing unit, and a mini conveyor type curing unit. When using the mini conveyer which was run with the conveyer stationary, the RM were exposed on a flat surface, while the cylindrical UV unit exposed RM on a curved surface.

The cylindrical UV exposure unit (Letralite, IKONICS Imaging, Duluth, MN, U.S.A.), utilized a $15 \mathrm{~W}$ fluorescent lamp that does not require warm up before use (Fig. 2.6). It is one of two units recommended by the RM manufacturer. After laying the transparent photomask on the $\mathrm{RM}$, the assembly was placed on a black flexible mat that was designed to tightly roll around the transparent cover, which kept a close contact between the photomask and RM during the exposure, and protected the user from being directly exposed to ultraviolet light.

The mini conveyor UV cure system (American Ultraviolet Company, Lebanon, IN, U.S.A.) used a fused quartz silicon dioxide curing lamp $(5.04 \mathrm{~W} / \mathrm{mm})$ shown in Fig. 2.7. The test samples were first placed on the conveyor and positioned directly under the UV lamp. Then, the conveyor was set to be stationary during the exposure of the RM samples. 


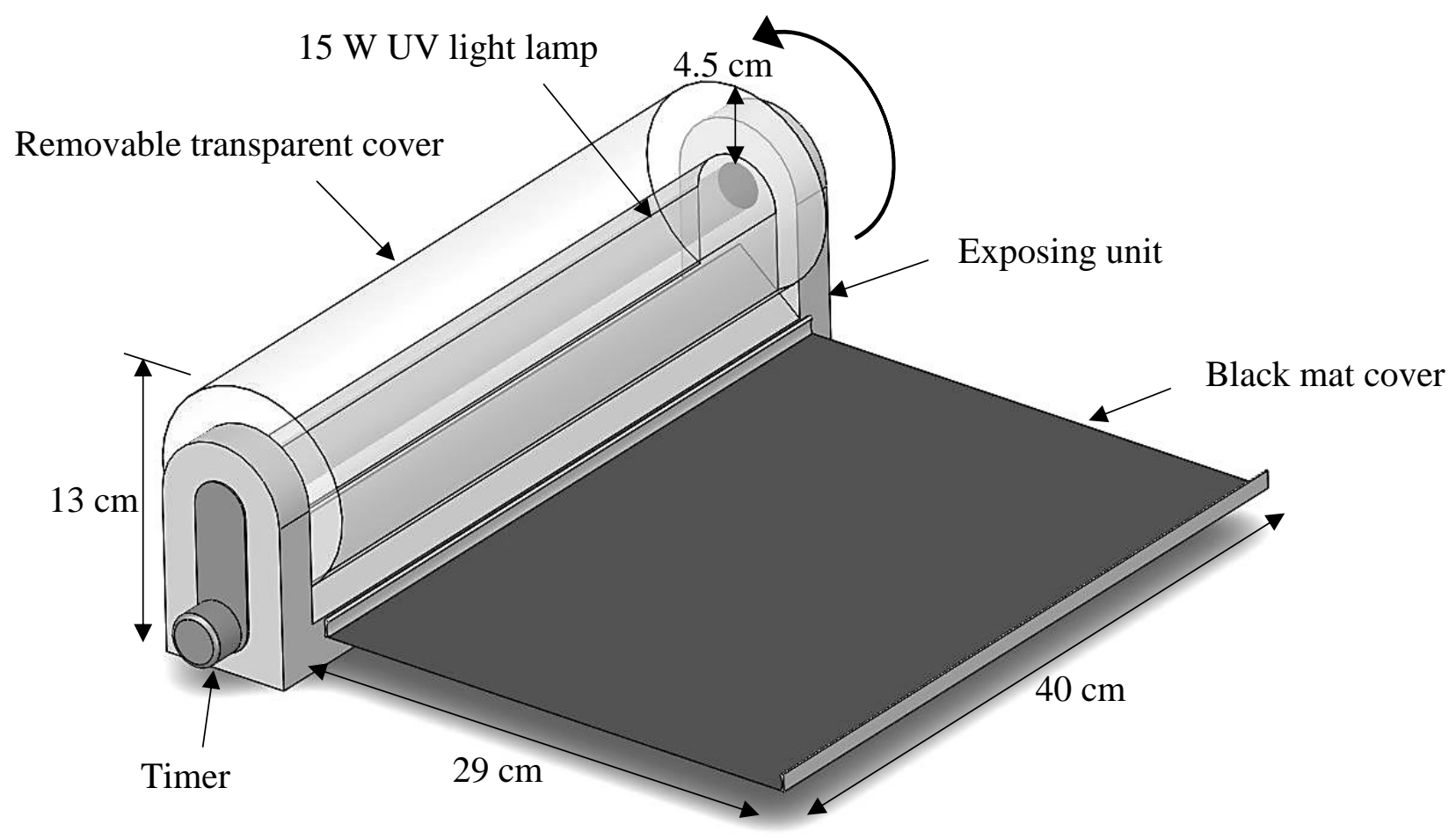

Figure 2.6 - Cylindrical ultraviolet exposure unit. 

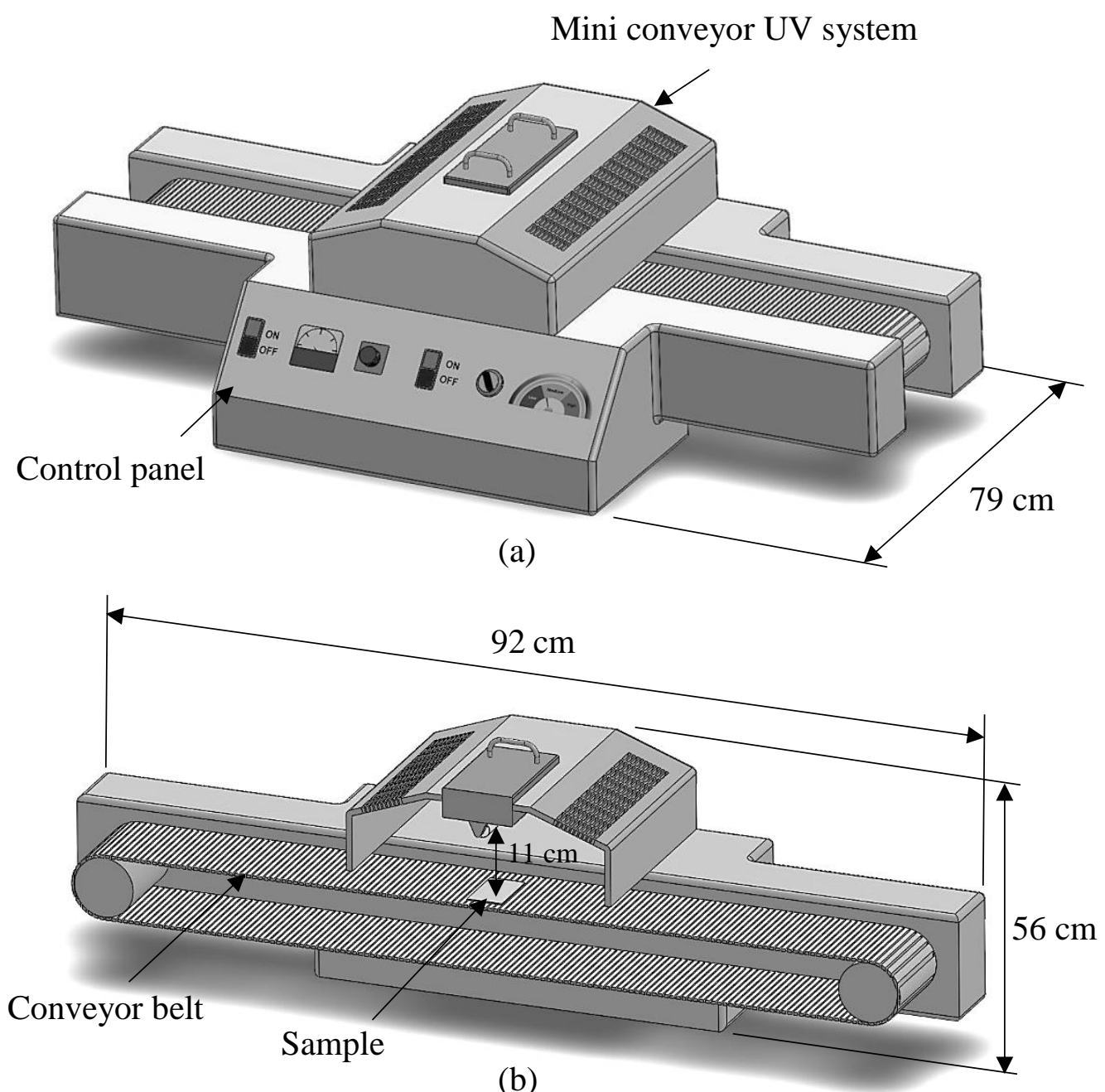

Figure 2.7 - Schematic of the mini conveyor UV exposure unit in (a) isometric view, (b) sectioned isometric view showing the placement of the samples and their distance from the lamp. The system was run with the conveyor stationary.

The UV radiation intensity in $\left(\mathrm{mW} / \mathrm{cm}^{2}\right)$ was measured at various locations within both of the units using an Accu-Cal 50 UV meter (Dymax Corporation, Torrington, CT, U.S.A.) that had a $9 \mathrm{~mm}$ diameter photo sensor (Fig. 2.8). For the cylindrical Letralite system, measurements were taken at various axial locations along the lamp, along the surface of the transparent cover, which was $\sim 4.5 \mathrm{~cm}$ from the source (Fig. 2.9). For the mini conveyor system, the intensity was measured directly below where the sample was exposed to UV light. The relation between the intensity and distance from the light source for both units was also measured by placing the measuring device on the lamp and moving it vertically ( $y$ direction in Figs. 2.9 and 2.10) away from it until the device read zero intensity. 


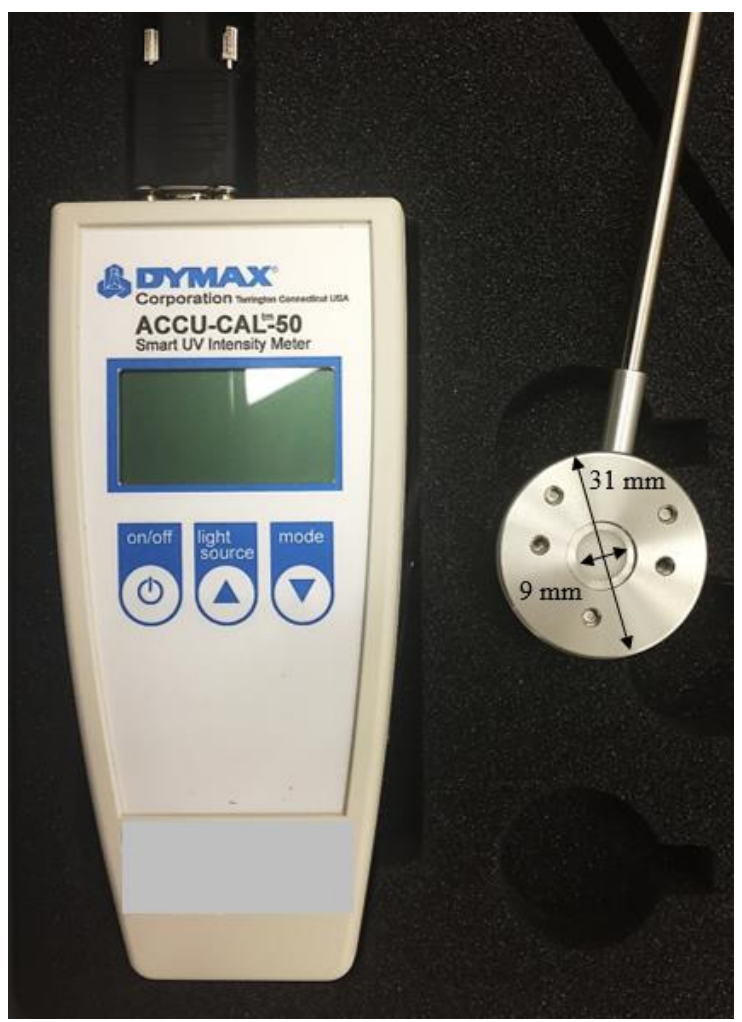

Figure 2.8 - Schematic of UV meter used to measure the UV radiation intensities.

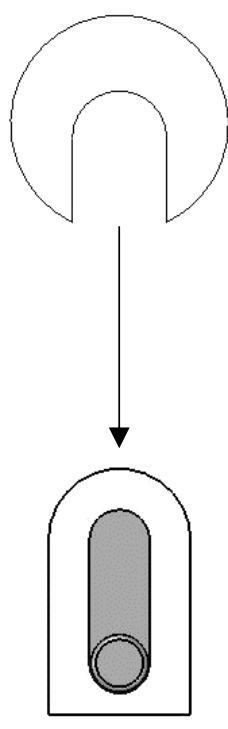

(a)

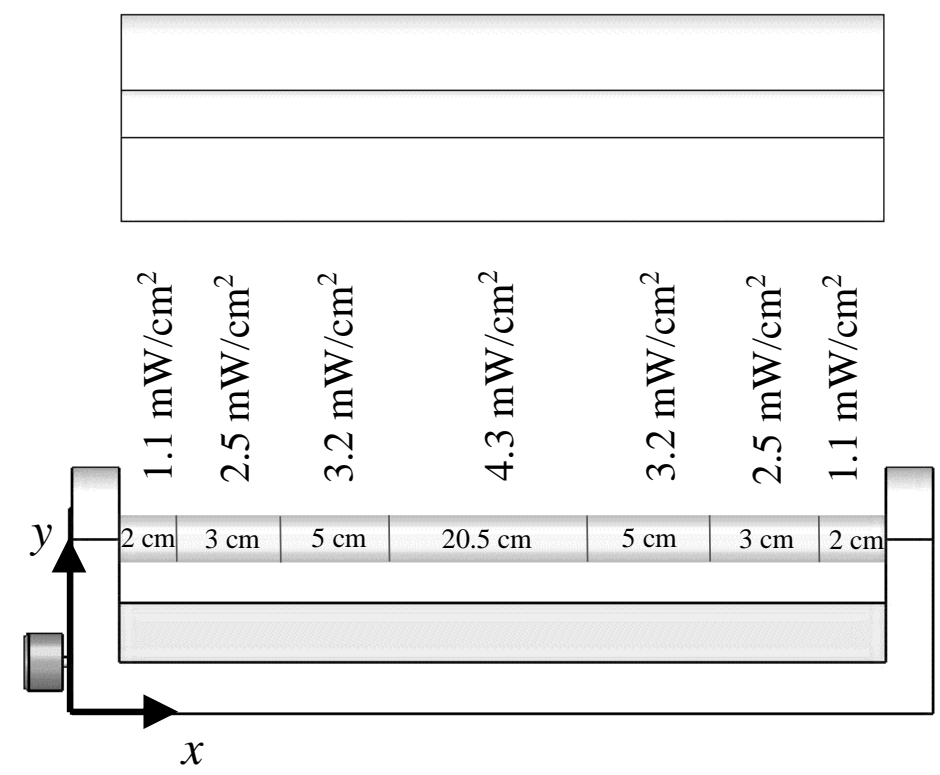

(b)

Figure 2.9 - Schematic of the cylindrical exposure unit in (a) side view, showing the placement of the transparent removable cover, and in (b) front view, showing the measured intensities along the length of the cylinder. 


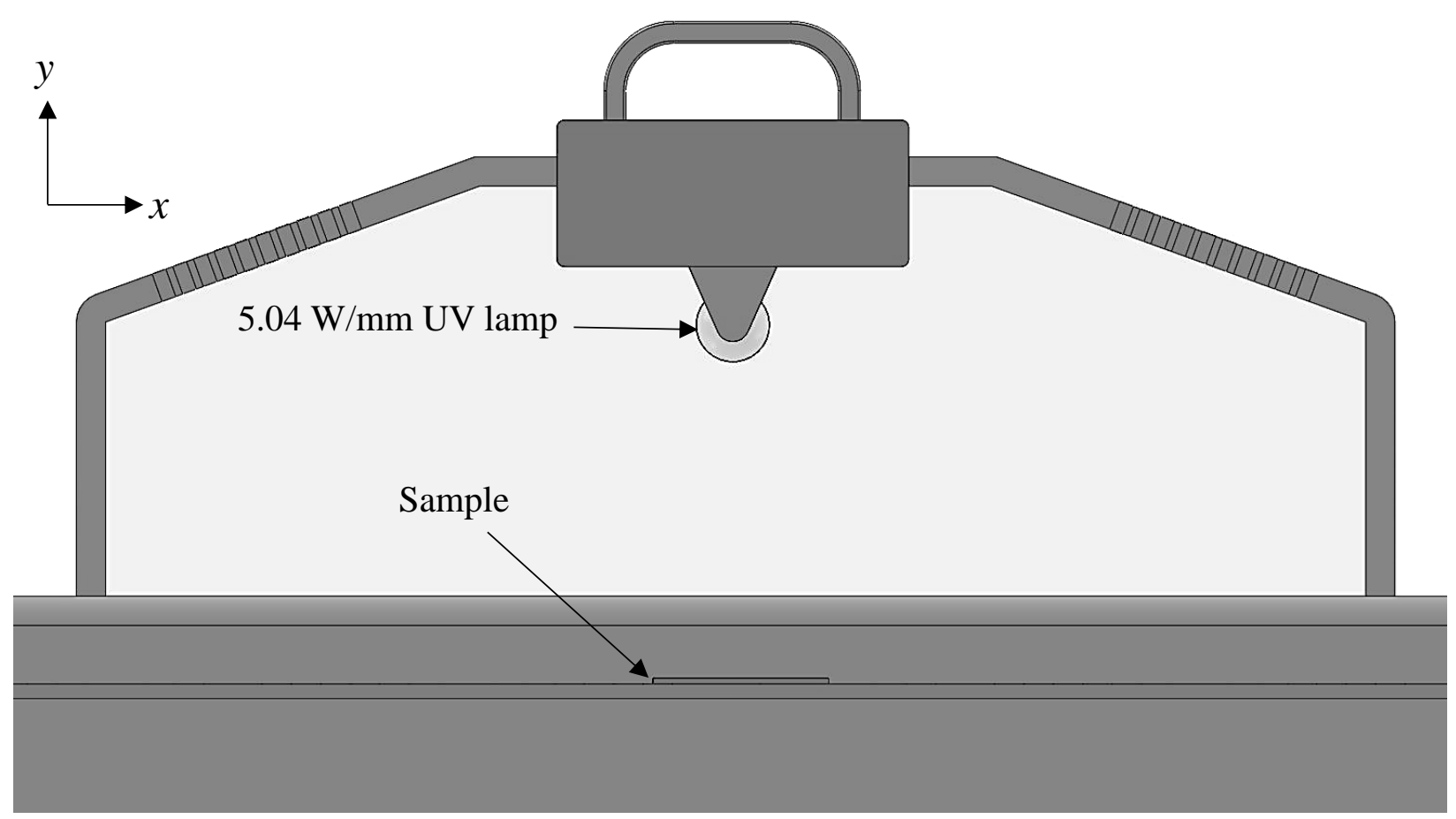

Figure 2.10 -Schematic of the mini conveyor unit showing the UV intensity and its region, shown in dotted lines with dimensions. The regions outside of the dotted areas are not exposed to UV light.

Since the manufacturer of the RM provided specifications for optimum cure only in terms of curing time for their recommended cylindrical unit, it was of interest to quantify the exposures of the RM to UV radiation in terms of a more fundamental measure, i.e., the energy density $\left(\mathrm{mJ} / \mathrm{cm}^{2}\right)$. The energy density corresponding to curing time recommended by the RM manufacturer was estimated by multiplying the measured UV intensity in the cylindrical cure unit by the recommended exposure time ( $120 \mathrm{~s}$ for $\mathrm{HT}$ and $90 \mathrm{~s}$ for HD). Table 1 shows that the energy densities using the RM manufacturer recommended exposure time of $120 \mathrm{~s}$ for HT RM varied significantly along the length of the surface of the cylinder, i.e., at the locations shown in Fig. 2.9. However, as shown in Fig. 2.9, $20.5 \mathrm{~cm}$ of the $40.5 \mathrm{~cm}$ total length had an approximately constant energy density of $516 \mathrm{~mJ} / \mathrm{cm}^{2}$. It was therefore assumed that this corresponded to the manufacturer recommended energy density for HT RM cure, and it was used as the baseline to compare the two systems. Similarly, the manufacturer recommended HD RM cure was estimated as $387 \mathrm{~mJ} / \mathrm{cm}^{2}$. 
Table 1 - UV light energy densities resulting from curing in the cylindrical Letralite unit for the manufacturer recommended exposure times for HT RM

\begin{tabular}{|c|c|c|c|}
\hline \multirow{4}{*}{$\begin{array}{c}\text { Cylindrical } \\
\text { Letralite }\end{array}$} & $\begin{array}{c}\text { UV light intensity } \\
\left(\mathrm{mW} / \mathrm{cm}^{2}\right)\end{array}$ & $\begin{array}{c}\text { Time } \\
(\mathrm{s})\end{array}$ & $\begin{array}{c}\text { UV light energy density } \\
\left(\mathrm{mJ} / \mathrm{cm}^{2}\right)\end{array}$ \\
\cline { 2 - 4 } & 4.30 & 120 & 516 \\
\cline { 2 - 4 } & 3.20 & 120 & 384 \\
\cline { 2 - 4 } & 2.50 & 120 & 300 \\
\cline { 2 - 4 } & 1.10 & 120 & 132 \\
\hline
\end{tabular}

To test the effect of sample location on the resulting pattern transfer, the patterned samples from Section 2.2.1 were cured in the cylindrical unit at the different locations shown in Fig. 2.9, which had the energy densities given in Table 1. The samples were placed such that the channel length was parallel to the axis of the cylinder unless otherwise mentioned.

In order to determine the effect of curing on a flat versus curved surface, HT RM samples in both systems were exposed to the same UV energy density i.e., in the conveyor system at 1.19 $\mathrm{mW} / \mathrm{cm}^{2}$ (Fig. 2.8) for $435 \mathrm{~s}$, to give the same energy density of $516 \mathrm{~mJ} / \mathrm{cm}^{2}$ as at the central portion of the Letralite system. The samples in the conveyor system were taped on a steel sheet which was painted dull black to minimize reflection of UV light. After measuring the width of the cured channel patterns from both curing systems, the RMs were attached to the borofloat glass samples, and then machined under identical conditions using AJM (Section 2.2.2). Regardless of which curing unit was used, for a particle mass flowrate of $\sim 8.5 \mathrm{~g} / \mathrm{min}$, five passes at a $1 \mathrm{~mm} / \mathrm{s}$ traverse speed were required to erode the brittle cured portions, before an additional pass eroded the glass to create a relatively shallow micro-channel.

\subsubsection{Effect of energy density}

To determine whether the pattern transfer was affected by UV energy density, the HT and HD RM patterned samples were cured within the cylindrical Letralite unit at a $4.30 \mathrm{~mW} / \mathrm{cm}^{2}$ intensity (Fig. 2.9) for between 60 and $360 \mathrm{~s}$, corresponding to energy densities between 258 and $1548 \mathrm{~mJ} / \mathrm{cm}^{2}$, respectively. The channel length was parallel to the axis of the Letralite cylinder during curing. The width of the cured channel patterns in the resulting masks were then measured. To determine the effects of curing energy density on the resulting eroded feature size, samples of the HT RM cured at 258, 516 (the manufacturer recommended), and $774 \mathrm{~mJ} / \mathrm{cm}^{2}$, and HD RM at 258 and $387 \mathrm{~mJ} / \mathrm{cm}^{2}$ (the recommended) were attached to the borofloat targets, and subjected to 
AJM using a particle mass flowrate of $\sim 8.70 \mathrm{~g} / \mathrm{min}$ and a $1 \mathrm{~mm} / \mathrm{s}$ transverse speed. The number of machining passes required to remove the cured portions of the RM generally increased for the undercured samples. For example, the $\mathrm{HT}$ at $258 \mathrm{~mJ} / \mathrm{cm}^{2}$ required seven passes, while at 516 , and $774 \mathrm{~mJ} / \mathrm{cm}^{2}$ only five passes were required. Similarly, the HD at $258 \mathrm{~mJ} / \mathrm{cm}^{2}$ required four passes, while at $387 \mathrm{~mJ} / \mathrm{cm}^{2}$, only 3 passes were required. After the brittle region of RM was removed, one additional pass was used to etch relatively shallow micro-channels into the glass.

\subsubsection{Effect of pattern orientation and location}

Since the cylindrical UV curing system introduced curvature in one direction during the cure, it was of interest to study whether the orientation of the channel patterns with respect to the curvature affected the pattern transfer. It was also of interest to test whether lateral or axial location made any difference. Therefore, two sets of channel patterns were cured for $120 \mathrm{~s}$ in the two different configurations shown in Fig. 2.11. All masks received the same $\sim 516 \mathrm{~mJ} / \mathrm{cm}^{2}$ energy density, except for Set 2 in Fig. $2.11 \mathrm{~b}$, at $\sim 384 \mathrm{~mJ} / \mathrm{cm}^{2}$ because it was further away from the cylinder center where the intensity was lower. All samples were then attached to borofloat glass and subjected to AJM with an $8.50 \mathrm{~g} / \mathrm{min}$ particle mass flow rate and a traverse speed of $1 \mathrm{~mm} / \mathrm{sec}$. All samples required five passes to remove the cured (brittle) region except for Set 2 of Fig. 2.11b, which required seven passes. All masks were then subject to a single pass to erode the borofloat glass substrates.

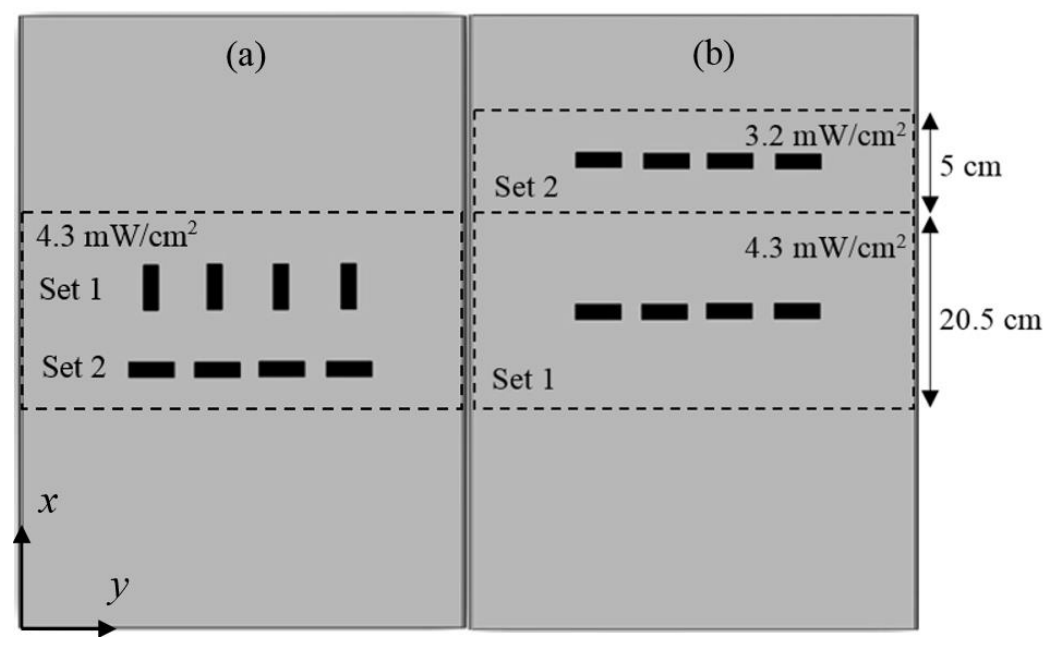

Figure 2.11 - Two sets of samples, with each box representing patterns of 250, 300, 400, 500, and $600 \mu \mathrm{m}$ wide channels, placed in the cylindrical UV curing system (Fig. 2.9) with channel length: (a) parallel and perpendicular to the cylinder $(x)$ axis; (b) perpendicular to the cylinder at 
different locations along the cylinder length. The curing lamp is parallel to the $x$ axis (Fig. 2.9(b)). The intensities are approximately constant within the zones shown within the dashed lines.

\subsubsection{Use of multiple RapidMask layers}

Ghobeity et al. [25] reported machining channels in glass that were $300 \mu \mathrm{m}$ wide and 150 $\mu \mathrm{m}$ deep before the HT RM fully eroded. To test whether multiple layers could be used to extend the mask lifetime and thus machine deeper (higher aspect ratio) channels, two single HT (100 $\mu \mathrm{m}$ thick) RM were stacked on top of each other creating a single $200 \mu \mathrm{m}$ thick mask, and patterned with the channels of Section 2.2.1 using $516 \mathrm{~mJ} / \mathrm{cm}^{2}$ of UV radiation. The orientation was with the channel length parallel to the cylinder axis, i.e. as for Set 1 in Fig. 2.11a. The test was repeated four times, and the average measured width of the cured portion was reported in Section 2.3.4.

To compare the width of the eroded features using a single layer HT RM and double layer HT RM, both cured samples were adhered to borofloat glass, and subjected to AJM under identical conditions, as described in Section 2.2.2 With a particle mass flowrate of $8.7 \mathrm{~g} / \mathrm{min}$, the cured patterns on the masks were fully eroded to the glass surface after five and ten passes at a $1 \mathrm{~mm} / \mathrm{s}$ transverse speed, for single and double layer HT RM, respectively. The samples were subjected to a further additional pass to create relatively shallow ( $\sim 30-70 \mu \mathrm{m}$ depending on channel width) channels, whose dimensions were measured with an optical profilometer. Then the channels were subjected to an additional four passes halfway along their length to establish whether higher aspect ratio channels could be made using the double layer system.

\subsubsection{Effect of side of mask exposed}

The RM mask has protective films on both sides that are removed before blasting, a "dull" adhesive side which is stuck to the target surface, and a "shiny" side that is ultimately exposed to abrasive particles. The manufacturer's instructions specify that the adhesive dull side should be exposed to the UV light $[61,62]$. Since it may be more convenient to first adhere the mask to the surface before curing it, it was of interest to investigate if curing the RM from the shiny side would affect the pattern transfer. Five samples of HT RM were exposed and patterned in the cylindrical unit on each side under identical conditions, with $516 \mathrm{~mJ} / \mathrm{cm}^{2}$ of UV light radiation, with the channel length parallel to the cylinder axis. One of the resulting HT RM cured through the shiny side and one cured through the dull side were then adhered to borofloat samples, and subject to 
AJM under identical conditions as described in Section 2.2.2. Both were first subjected to five passes at $1 \mathrm{~mm} / \mathrm{s}$ scan speed to erode the cured brittle RM, and a further single pass to erode the masked glass.

\subsubsection{Erosion rate of uncured, cured RM, and borosilicate glass}

The volumetric erosion rate, i.e. the volume of target material removed per unit mass of incident erodent, was measured on bulk samples of both the uncured and cured $\left(516 \mathrm{~mJ} / \mathrm{cm}^{2}\right) \mathrm{RM}$ material, and on a borofloat glass sample that had been patterned with 400 and $500 \mu \mathrm{m}$ channels using the HT mask material. The experiments were performed under the same conditions as Section 2.2.2, but with the nozzle inclined to the surface at various angles between 20 and $90^{\circ}$. The plane of inclination was parallel to the axis of the channels so that symmetric channels were

formed. Such standard erosion tests are useful to establish material removal mechanisms, and can be used to provide guidelines regarding the efficacy of the mask under various process conditions. For all tests, the target was scanned at a constant speed of $1 \mathrm{~mm} / \mathrm{s}$ over a length of $10 \mathrm{~mm}$. The erosion rate was then obtained from the measured volume of the eroded regions obtained using the optical profilometer, the particle mass flow rate $(9.0 \mathrm{~g} / \mathrm{min})$, and the machining time $(10 \mathrm{~s})$. 


\subsection{Results and Discussion}

\subsubsection{Effect of UV curing unit}

Figure 2.12 shows that the measured intensities were similar for the two curing units, decreasing sharply with increasing vertical distance (along $y$ in Fig. 2.9 and Fig. 2.10) from the source. The locations where the RM samples were cured directly below the light are also shown. The UV radiation intensity in the mini-conveyor unit was not proportional to $1 / r^{2}, r$ being the distance from the UV source point, which may be attributed to reflections within the chamber of the unit.

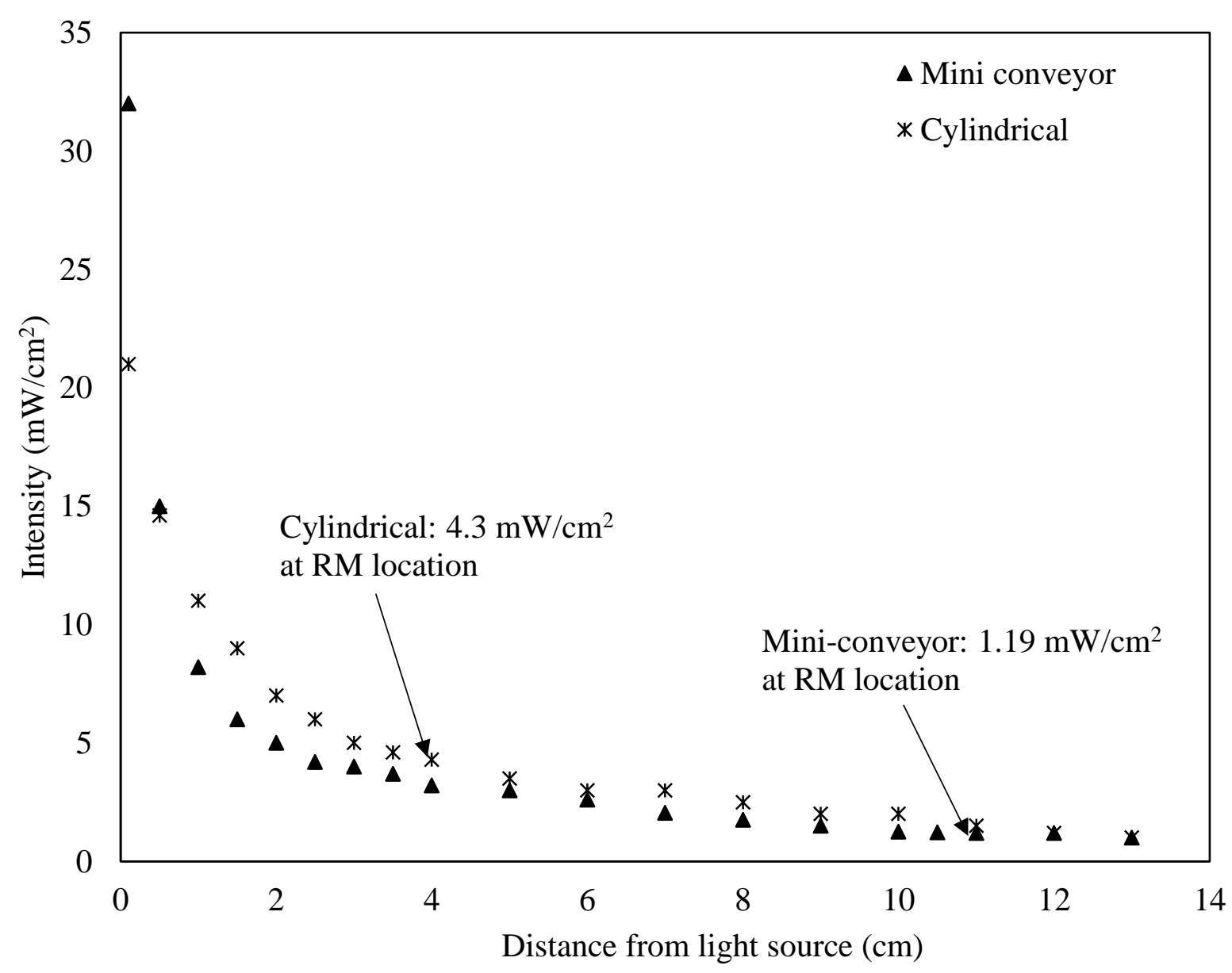

Figure 2.12- Intensity measurement, in $\mathrm{mW} / \mathrm{cm}^{2}$, of the cylindrical and mini-conveyor exposure units at various $y$ distances from the source. The position of the mask during curing in each unit is indicated.

Figure 2.13 shows the 'pattern transfer accuracy', i.e. the width of the cured portion of the mask compared to the 'design width' of the patterns on the transparency photomask, for the HT 
RM using both the flat mini conveyor and the cylindrical UV light exposing systems at the same recommended energy density of $516 \mathrm{~mJ} / \mathrm{cm}^{2}$. As expected, regardless of design channel width and which curing unit was used, the cured channel widths were consistently between $\sim 15 \mu \mathrm{m}$ and $\sim 20$ $\mu \mathrm{m}$ larger than design, i.e. as long as the RM samples were sufficiently UV cured, the pattern transfer accuracy was the same.

Figure 2.13 also shows the width of the relatively shallow $(\sim 40-60 \mu \mathrm{m}$ in depth) channels that were machined into glass using the HT RM samples that were cured in both the cylindrical and mini-conveyor systems. The widths of the actual AJM eroded channels were similar for both units, about 15-20 $\mu \mathrm{m}$ less than the width of the transferred patterns, and within $\sim 3 \%$ of the design. The narrower width was expected since particles arriving within one particle radius $(\sim 12.5 \mu \mathrm{m}$ on average) of the mask edge will hit it and thus not reach the surface. Indeed, as shown in Fig. 2.3, a small portion at the edges of the cured RM remains untouched by particle impacts. In summary, curing on a flat or curved surface did not affect the resulting feature width.

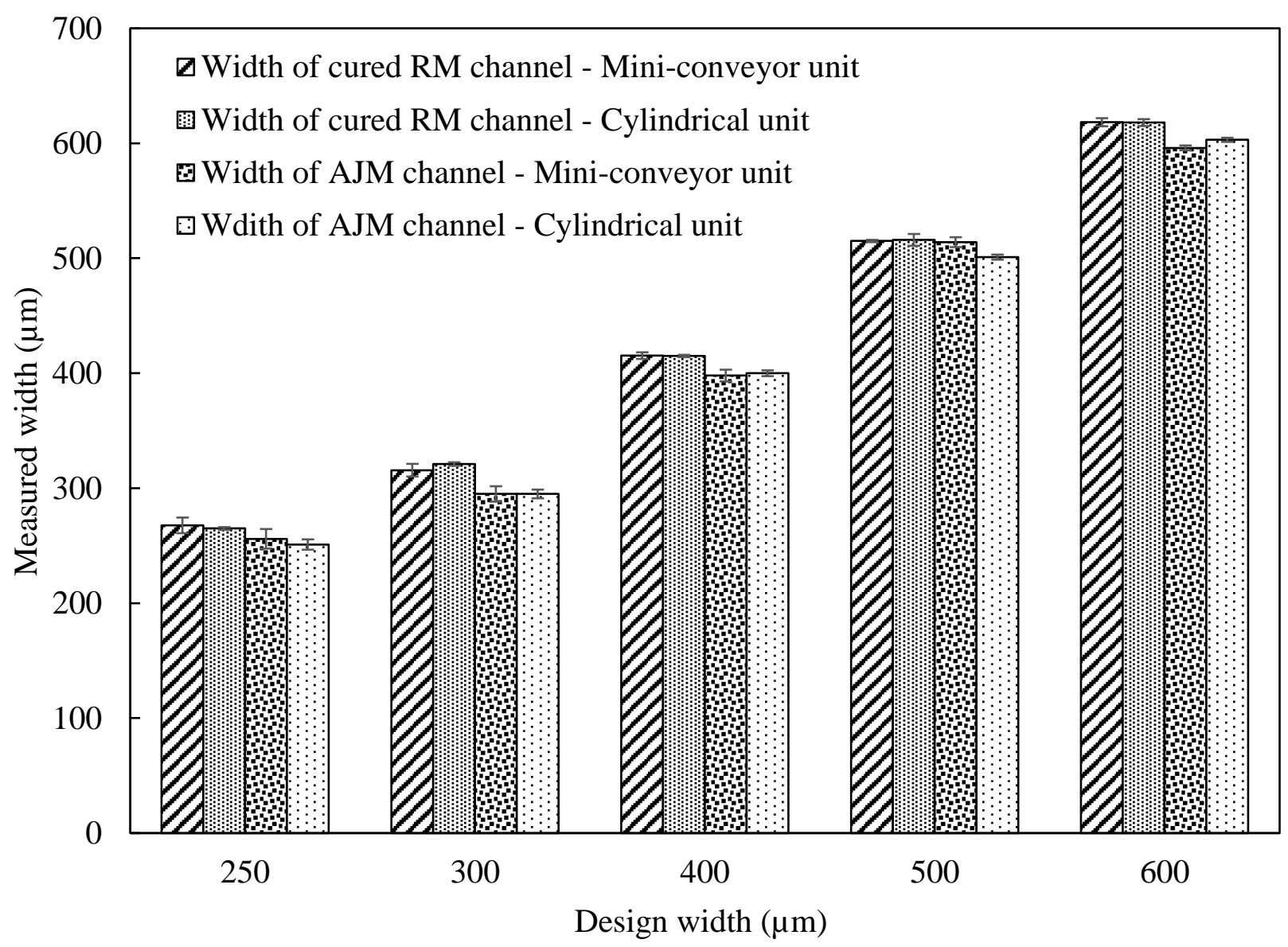

Figure 2.13 - Measured widths of cured HT RM and AJM channels for 5 different design widths using the mini-conveyor curing system and cylindrical UV unit at the same energy density of 
$516 \mathrm{~mJ} / \mathrm{cm}^{2}$. Error bars indicate the standard deviation of the results of 6 sets of experiments for the UV curing process and 3 sets of experiments for the AJM process.

\subsubsection{Effect of energy density}

The manufacturer instructions indicated that overexposing the RMs should not significantly affect their performance, while underexposing them could compromise their performance [61,62]. Figures 2.14 and 2.15 show that the widths of the cured patterns increased with increasing energy density for both thicknesses of RM and that near the optimum recommended curing conditions, the cured widths were similar. For example, at $516 \mathrm{~mJ} / \mathrm{cm}^{2}$ (the recommended) and $774 \mathrm{~mJ} / \mathrm{cm}^{2}$, the pattern transfer to the HT RM were $\sim 20-30 \mu \mathrm{m}$ larger than design (Fig. 2.14). Similarly, for the HD RM, the cured widths at the optimum $387 \mathrm{~mJ} / \mathrm{cm}^{2}$ and $516 \mathrm{~mJ} / \mathrm{cm}^{2}$, were both $\sim 10-20 \mu \mathrm{m}$ larger than design (Fig. 2.15). However, for the samples cured at higher energy densities, i.e. 1032 and $1548 \mathrm{~mJ} / \mathrm{cm}^{2}$ for the HT, and $774 \mathrm{~mJ} / \mathrm{cm}^{2}$ for the HD, the transferred patterns became significantly (60-90 $\mu \mathrm{m}$ for HT, and 50-60 $\mu \mathrm{m}$ for HD) larger than design. This indicates that, although some degree of overcuring can be tolerated, at sufficiently high energy densities, the channels will become significantly larger than desired. Figures 2.14 and 2.15 also indicate that undercuring generally results in cured pattern transfer to the mask that is very close to design. For example, for both the HT and HD RM undercured at $258 \mathrm{~mJ} / \mathrm{cm}^{2}$, the width of the transferred patterns were only $\sim 1-5 \mu \mathrm{m}$ larger than the design width. 


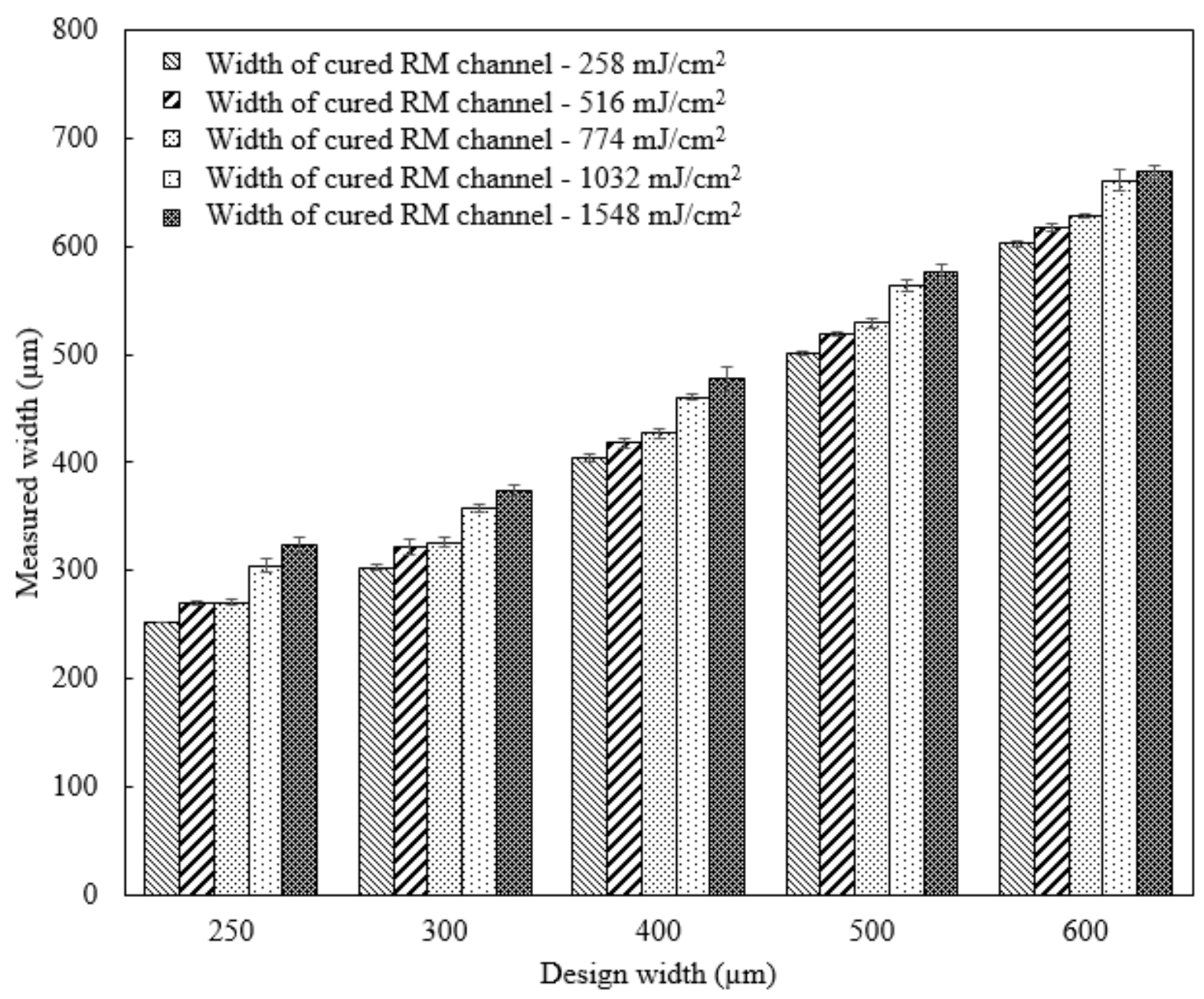

Figure 2.14 - Measured widths of HT RM cured at energy densities of 258, 516, 774, 1032, and $1548 \mathrm{~mJ} / \mathrm{cm}^{2}$. Error bars indicate one standard deviation of 4 sets of experiments. 


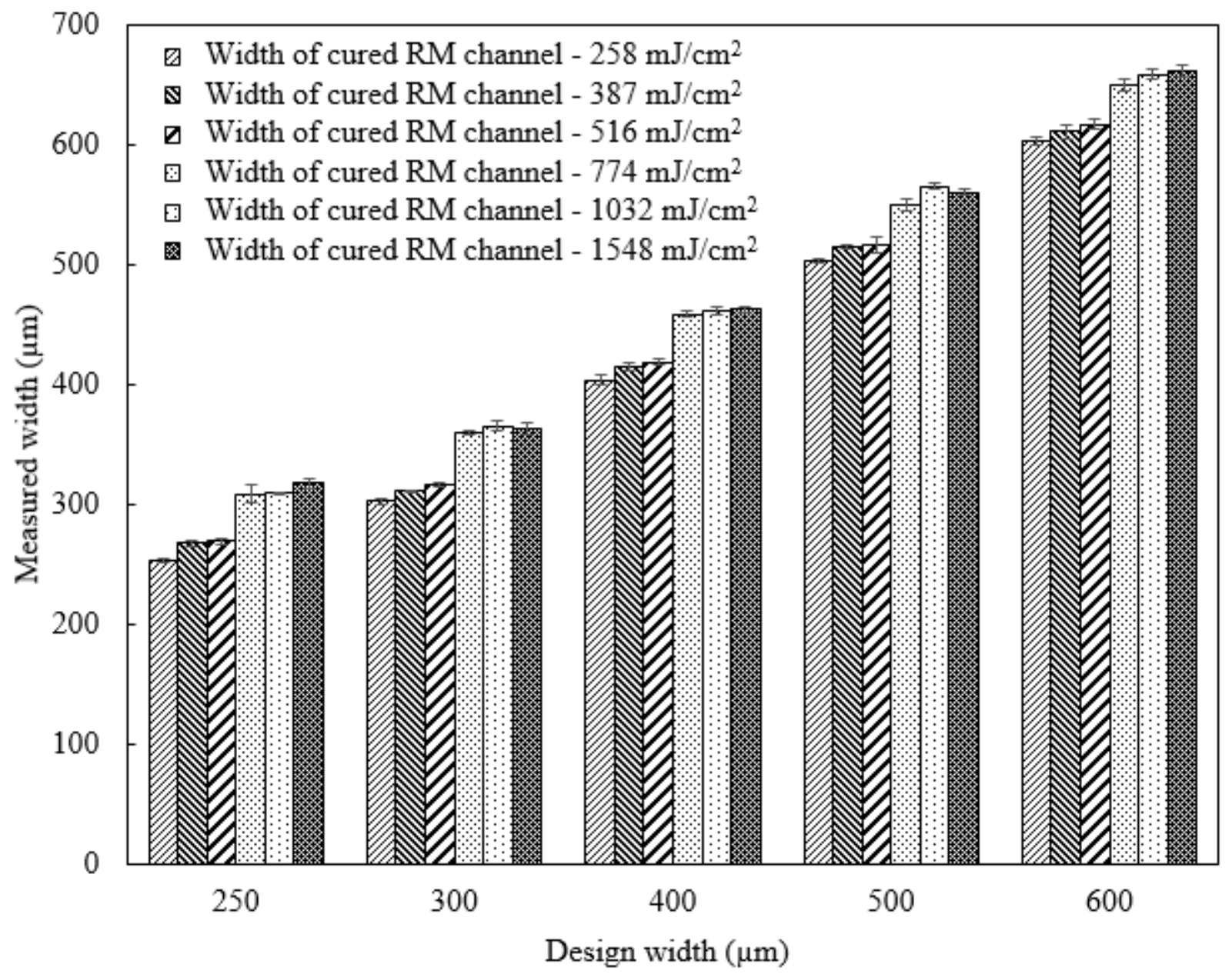

Figure 2.15 - Measured widths of HD RM cured at energy densities of 258, 387, 516, 774, 1032, and $1548 \mathrm{~mJ} / \mathrm{cm}^{2}$. Error bars indicate one standard deviation of 4 sets of experiments.

The apparent advantage of improved pattern transfer by undercuring was negated by the increased particle dose required to remove the undercured mask material, which, in turn, decreased the cured mask durability. Figure 2.16 shows the cured HT RM channel and AJM eroded channel widths at 258, 516 and $774 \mathrm{~mJ} / \mathrm{cm}^{2}$. As mentioned in Section 2.2.3, under identical conditions, five AJM passes were required to fully remove the cured mask materials at 516 and $774 \mathrm{~mJ} / \mathrm{cm}^{2}$, while eight passes were required at the undercured $258 \mathrm{~mJ} / \mathrm{cm}^{2}$. Similarly, the HD RM at 387 $\mathrm{mJ} / \mathrm{cm}^{2}$ required three passes to remove the brittle region of RM, while it took four passes at 258 $\mathrm{mJ} / \mathrm{cm}^{2}$. This was clear evidence of a critical minimum energy density required to fully cure the RM. As shown in Figs. 2.16 and 2.17, the longer required machining time associated with the undercured RM also ultimately resulted in significantly wider channels than designed (average of $\sim 15 \%$ wider for $\mathrm{HT}$ and $4 \%$ for $\mathrm{HD}$ ). In contrast, the RM cured at the recommended energy 
densities or slightly above ultimately resulted in channel widths that were much closer to design (within 5\% for HT, and 1\% for HD).

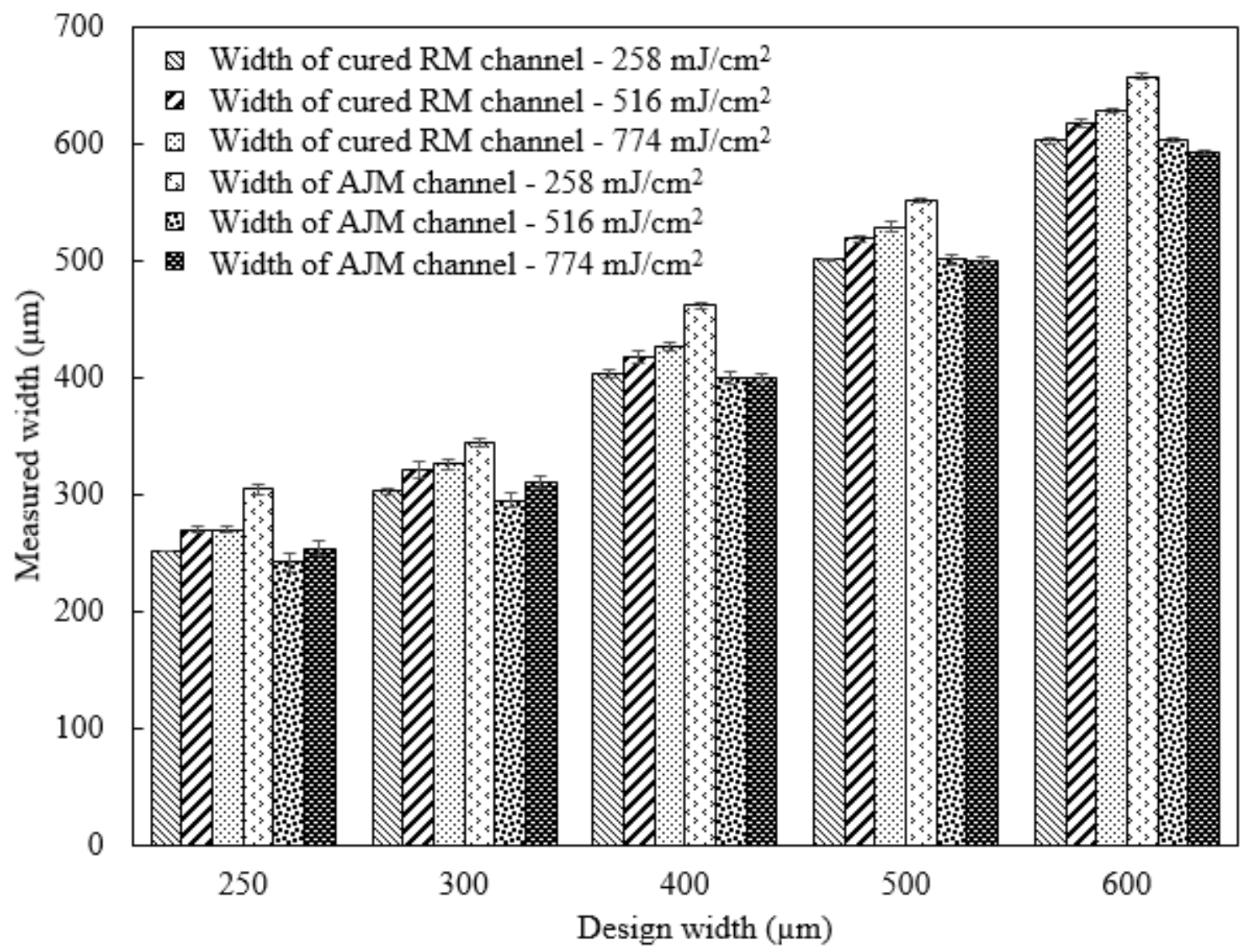

Figure 2.16 - Measured widths of cured HT RM and resulting AJM channel widths when curing at three energy densities. Error bars indicate one standard deviation of for 4 sets of experiments for UV exposure and 3 sets of AJM process.

In summary, curing at $387-516 \mathrm{~mJ} / \mathrm{cm}^{2}$ for the HD RM, and $516-774 \mathrm{~mJ} / \mathrm{cm}^{2}$ for the HT RM, gave the best overall results in terms of eroded feature width. Although the manufacturer did not specify a recommended curing energy density, these values are roughly consistent with the curing times that they recommended for their specific curing unit. Energy densities out of these ranges will either result in premature wear of the masks or features that are significantly wider than desired. 


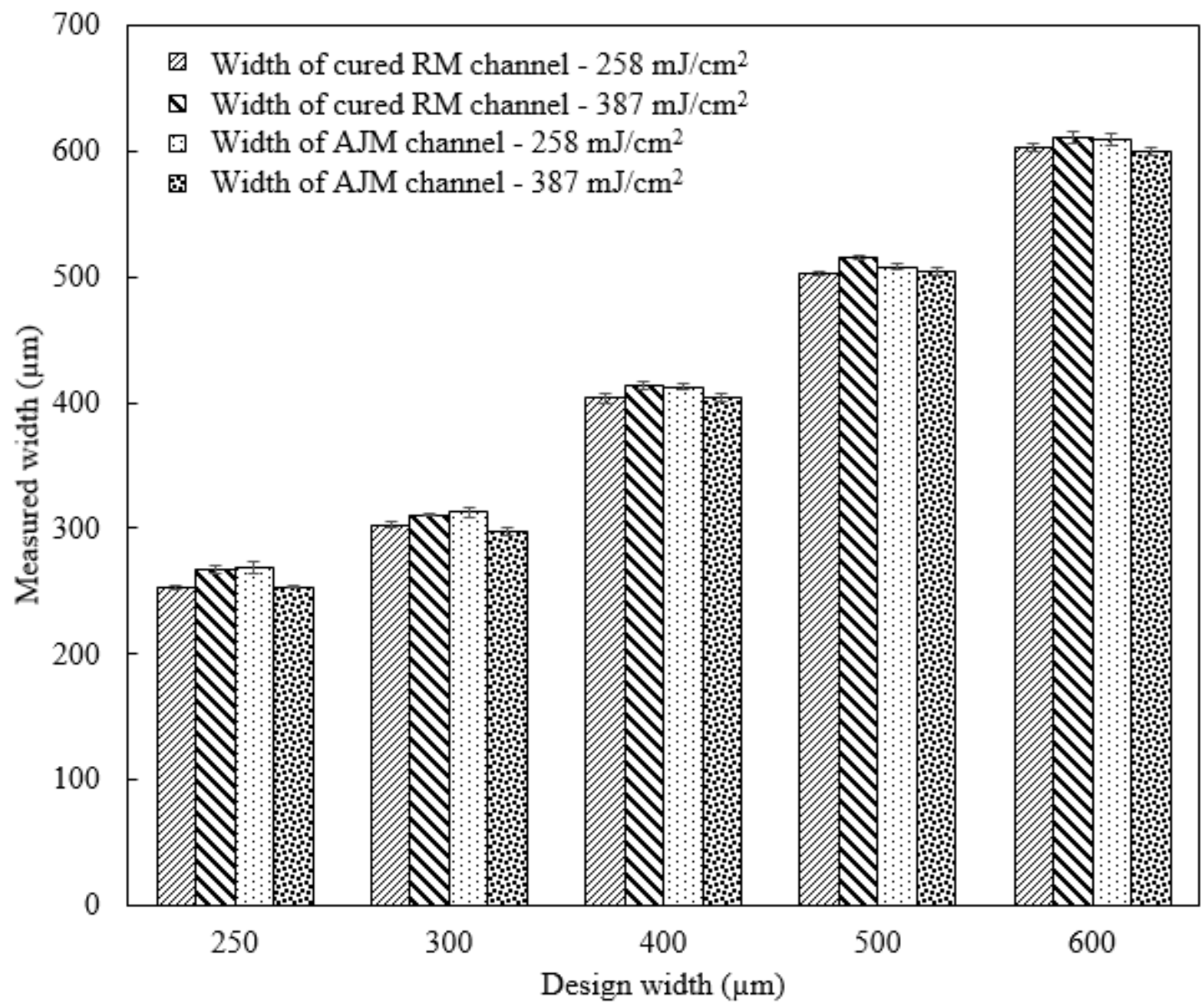

Figure 2.17 - Measured widths of cured HD RM and resulting AJM channel widths when curing at two energy densities. Error bars indicate one standard deviation of for 4 sets of experiments for UV exposure and 3 sets of AJM process.

\subsubsection{Effects of pattern orientation and location within cylindrical unit}

Figure 2.18, corresponding to curing in the orientation in Fig. 2.11a, shows that the effect of the orientation of the channels, i.e. whether the channel length was parallel or perpendicular to the axis on the UV light source, while curing in the cylindrical unit was insignificant $(P>0.05)$. 


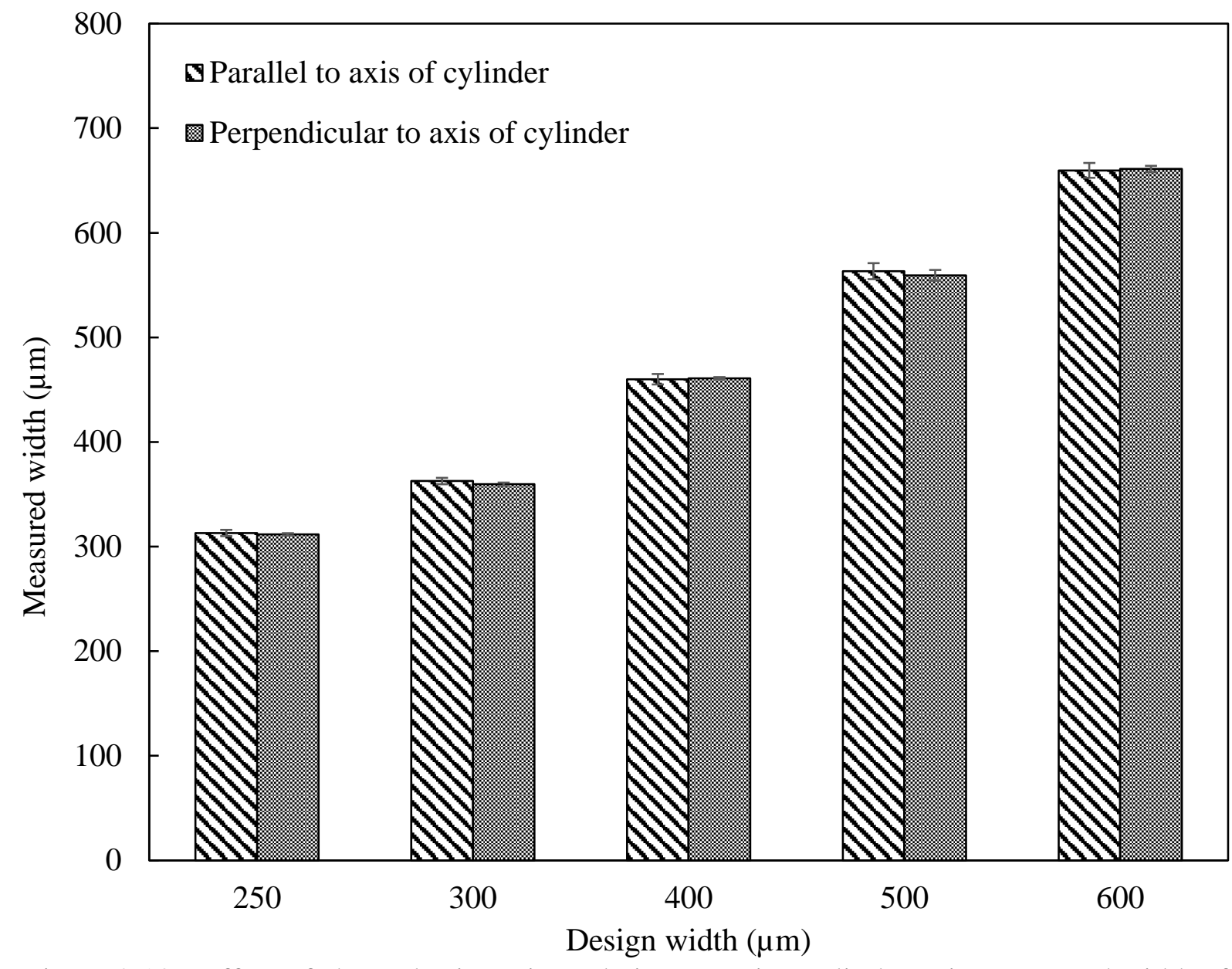

Figure 2.18 - Effect of channel orientation relative to curing cylinder axis. Measured width of cured region of HT RM for 5 different channel widths exposed in cylindrical unit at energy density $516 \mathrm{~mJ} / \mathrm{cm}^{2}$. The samples were placed perpendicular and parallel to the axis of the cylinder as in Fig. 2.11a. Error bars indicate one standard deviation of 4 sets of experiments.

As expected, since the UV light energy density was constant around the circumference of the cylinder at any point along the axis of the cylinder, the y-coordinate of the location of the samples had no effect on the pattern transfer. However, Figure 2.9(b) shows that there was a variation in measured intensities along the length ( $x$ coordinate) of the cylindrical Letralite unit, resulting in different energy densities along the axis of the lamp for the same curing time. Figure 2.19, corresponding to the curing configuration in Fig. 2.11b, shows that the RM samples cured near the center of the cylinder length at the recommended energy density $\left(516 \mathrm{~mJ} / \mathrm{cm}^{2}\right)$ have eroded features geometries closer to the design width ( within $3 \%$ of design width) than eroded channels resulting from the RM samples cured and the ends of the cylinder, where the energy density was $384 \mathrm{~mJ} / \mathrm{cm}^{2}$ ( $15 \%$ wider than design width), evidence of undercured RM. Therefore, 
it is recommended to place the RM samples within the middle $20.5 \mathrm{~cm}$ range of the UV light unit shown in Fig. 2.9b to achieve sufficient curing.

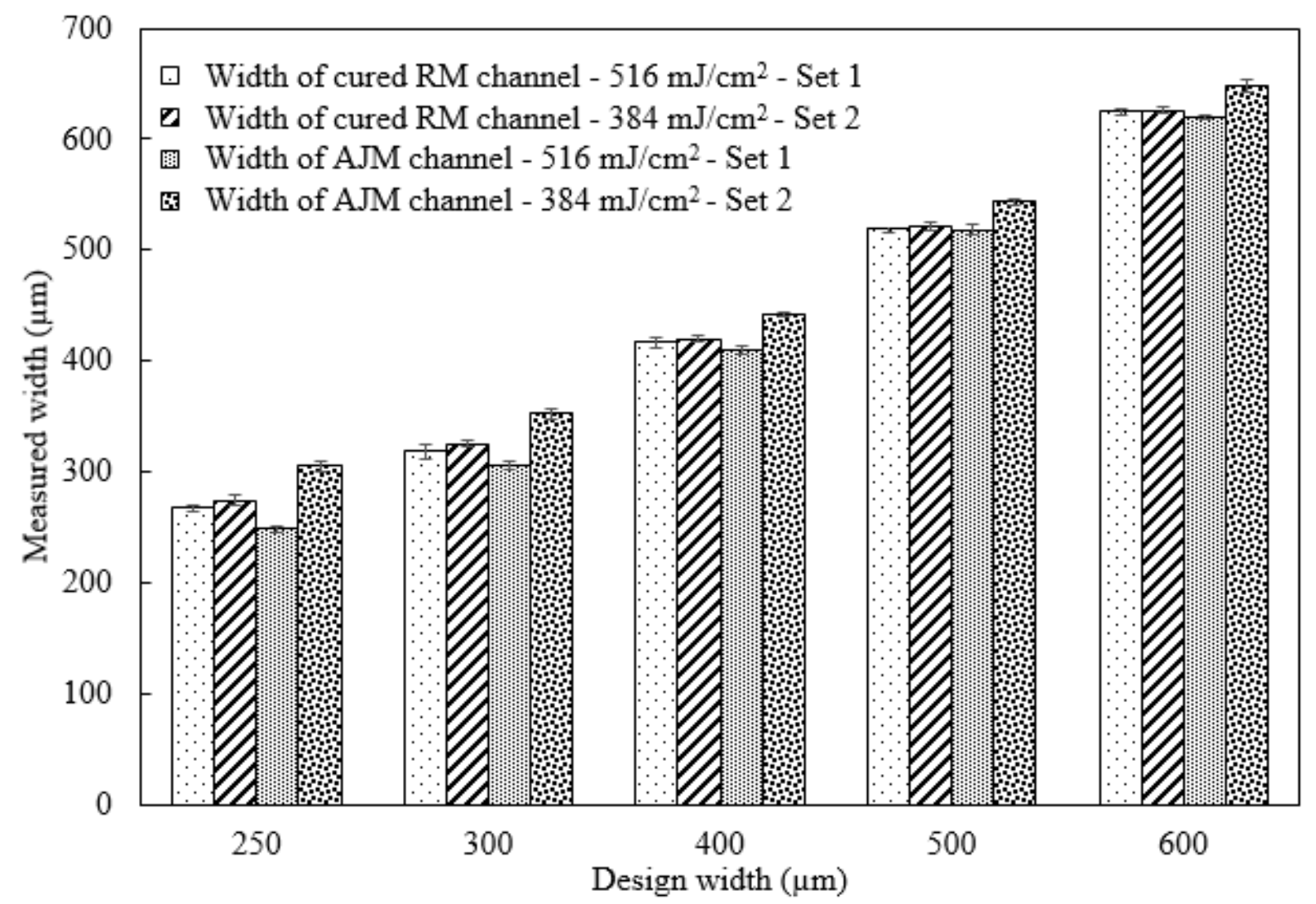

Figure 2.19 - Measured widths of cured HT RM and resulting AJM channel widths cured at two energy densities, 384 and $516 \mathrm{~mJ} / \mathrm{cm}^{2}$. The samples were placed with orientations shown in Fig. 2.11b. Error bars indicate one standard deviation from 4 sets of experiments for UV exposure and 3 sets of experiments for AJM process.

\subsubsection{Effect of multiple RapidMask layers}

As explained in Section 2.2.6, a novel method was introduced to expose multiple RM layers as a single thicker mask, in an attempt to increase the erosion resistance, and ultimately improve the achievable aspect ratio. Both single and double were UV exposed at the same energy density of $516 \mathrm{~mJ} / \mathrm{cm}^{2}$ in order to compare the two under identical conditions. Figure 2.20 shows that the double layer system did not significantly change the accuracy of the pattern transfer, i.e. the width of the cured patterns were within $\sim 3 \mu \mathrm{m}$ of each other. It took five passes to erode the cured regions of the single layer HT RM down to the glass, while ten passes were required for the double layer RM since the thickness was twice as large. Figure 2.20 also shows that, under identical conditions, 
the double layer HT RM resulted in narrower shallow channels, i.e. channels eroded with a single layer were an average of $\sim 5 \%$ narrower than design, whereas channels machined with a double layer were $\sim 3 \%$ wider than design. The reason for the narrower channel when using the thicker mask is likely related to differences in the trajectories of ricocheting particles. As explained by Nouhi et al. [17], when thicker masks are used, more particles tend to ricochet from the mask edge and strike the opposite side of the mask, rather than ricocheting onto the surface, as occurs with thinner masks. This leads to a lower erosion rate at the channel edges when thicker masks are used.

Figure 2.20 shows that the double layer HT RM resulted in narrower deep channels, under identical machining conditions, i.e., channels eroded with a single layer were an average of $\sim 13 \%$ wider than design, whereas channels machined with a double layer were $\sim 3 \%$ wider than design. The reason for the larger channel using a thinner mask likely was due to more rapid erosion of the mask edges. This hypothesis is supported by the increased durability observed for the double layer system. The manufacturing recommended curing time for $100 \mu \mathrm{m}$ thick HT RM was $129 \mathrm{~mJ} / \mathrm{cm}^{2}$ more than the UV energy density needed for the thinner, $50 \mu \mathrm{m}$ thickness, HD RM. Extrapolating from this, it is recommended to cure the $200 \mu \mathrm{m}$ thick double layer HT RM at between 516 and $645 \mathrm{~mJ} / \mathrm{cm}^{2}$. 


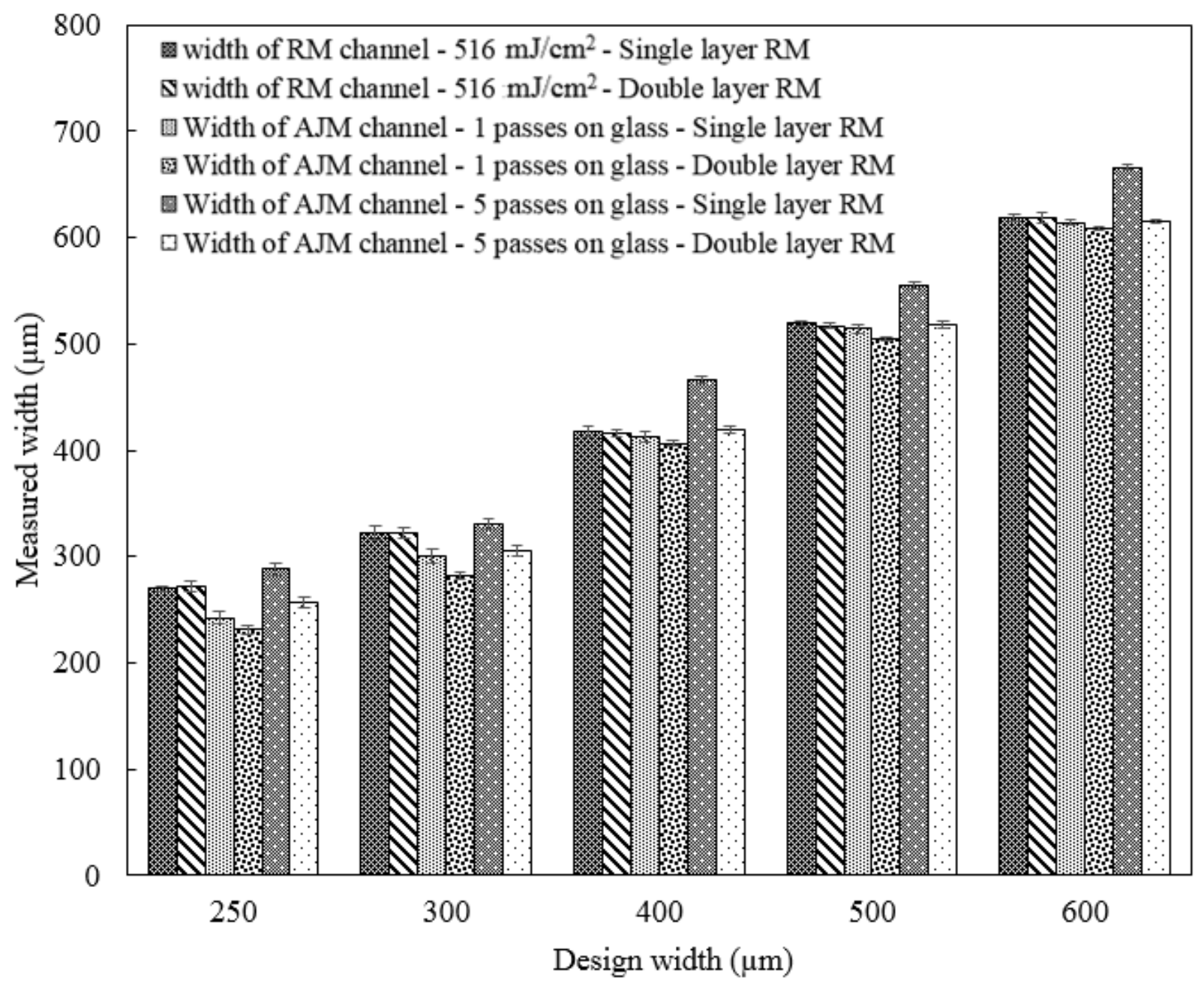

Figure 2.20 - Measured widths of cured single and double HT RM and resulting AJM channel widths exposed in the cylindrical UV exposure unit at an energy density of $516 \mathrm{~mJ} / \mathrm{cm}^{2}$. Error bars indicate one standard deviation of 4 sets of experiments for UV exposure and 3 sets of AJM process.

Figure 2.21 shows the resulting average measured centerline depth of the eroded shallow channels. The average centerline depths were $~ 8 \%$ deeper when the double layer HT RM was used, when compared to single layer RM, after 1 pass, and $\sim 16 \%$ deeper after 5 passes. These differences were even more significant for narrower channels. Channels with widths of 250 and $300 \mu \mathrm{m}$ resulted in $\sim 30 \%$ larger average depth using the double layer RM, when compared to single layer RM, after 5 passes of AJM. The deeper channels resulting from using the thicker mask are likely due to particle ricochet and second strike effects. As explained by Slikkerveer et al. [16] and Ghobeity et al. [67], steep channel walls, or thicker masks, cause particles to rebound from the 
channel or mask sidewalls and strike the center of the channel, thus providing additional erosion at that location.

After 1 pass of AJM on glass, the average aspect ratios of the eroded channels for both samples were between 0.15 and 0.20 . After 5 passes of AJM, the average aspect ratios of the eroded channels were $\sim 0.40$ when the single layer HT RM was used, and this value improved to 0.56 for eroded channels using the double layer RM. This, and the increased mask durability resulting from using multiple RM layers, implies that use of multiple layers is effective in increasing the maximum possible achievable aspect ratio.

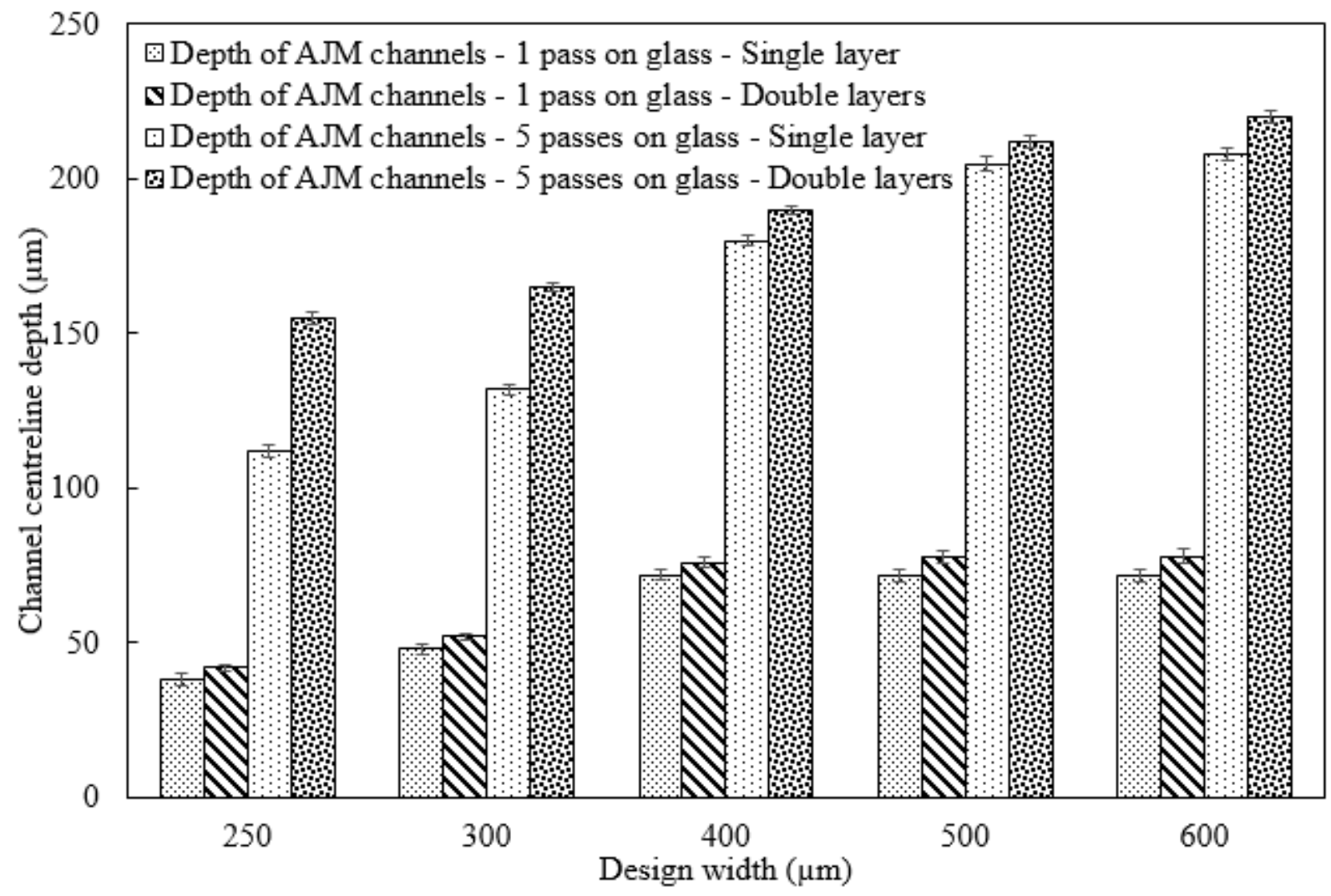

Figure 2.21 - Centerline depth of AJM channels machined using single and double HT RM for 1 and $5 \mathrm{AJM}$ passes on the glass. Error bars indicate one standard deviation from 4 measurements of eroded channels.

\subsubsection{Effect of side of mask exposed}

Figure 2.22 shows that exposing the RM from either the dull or shiny side did not significantly affect either the width of the cured region $(P>0.05)$ or the width of the eroded channel $(P>0.05)$. Therefore, it is feasible to first adhere the RM onto the glass samples, UV expose it, and 
then machine it using AJM. For flat-bed UV curing systems, this could offer significant time savings since the preparation time would be reduced.

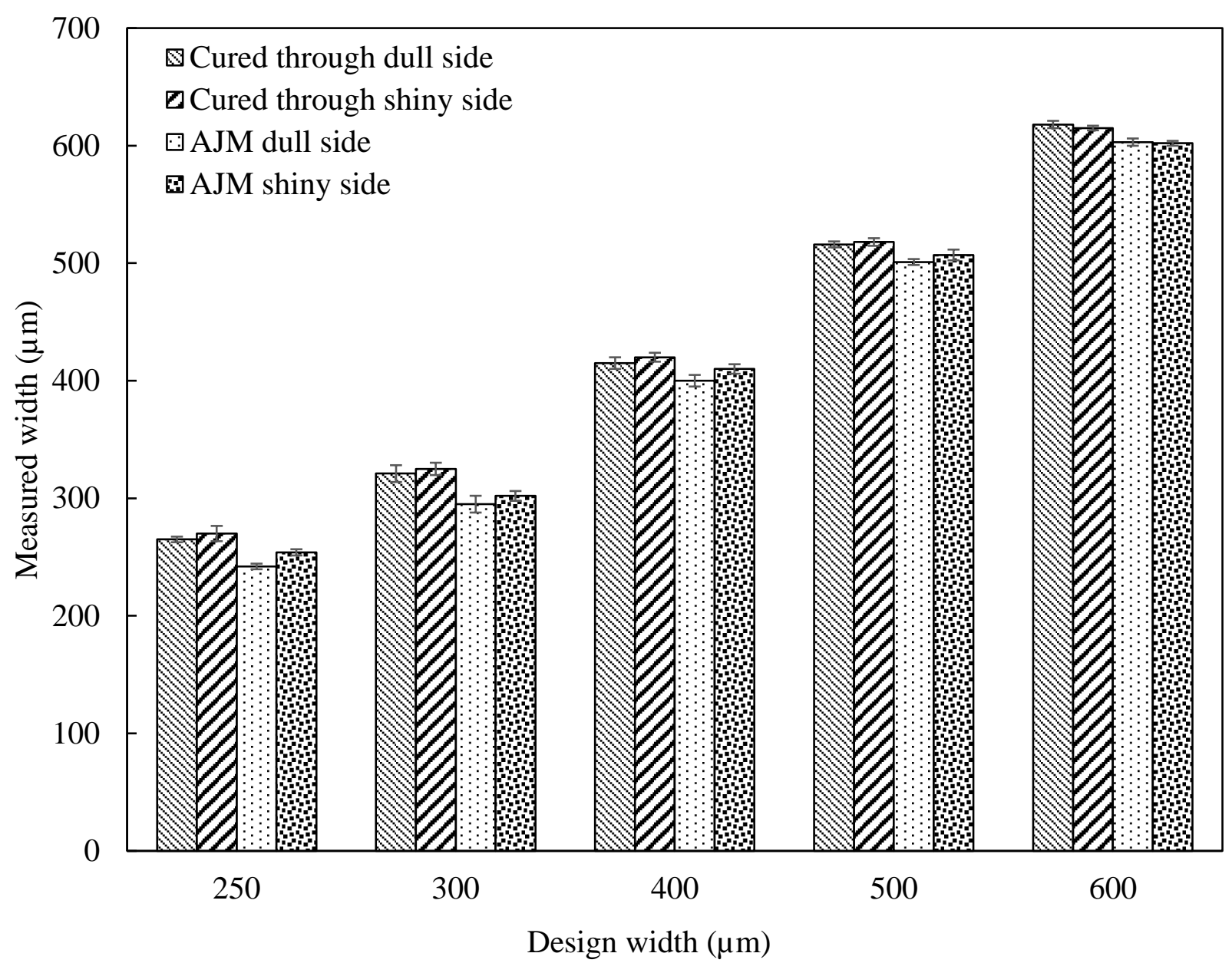

Figure 2.22 - Effect of curing the RM from dull and shiny sides. Measured width of cured region and resulting AJM channel widths of HT RM for 5 different channel widths exposed in cylindrical unit at UV energy density $516 \mathrm{~mJ} / \mathrm{cm}^{2}$. The samples were exposed from the dull and shiny side and were placed with set 1 orientation shown in Fig. 2.11b. Error bars indicate one standard deviation of 4 sets of experiments for UV exposure and 3 sets of AJM process.

\subsubsection{Erosion rate of Uncured RM, cured RM, and borosilicate glass}

The relative erosion rates of the uncured mask, the cured mask, and the target material play an important role in the AJM process. In general, the erosion behaviour of most materials can be classified as either 'brittle', characterized by a maximum erosion rate at a perpendicular incidence, or 'ductile', characterized by a maximum erosion rate at oblique incidence [30]. Figure 2.23 shows the normalized erosion rate of uncured, UV cured HT RM exposed at the manufacturer 
recommended energy density, and borosilicate glass when machined with AJM. Each curve was normalized to its corresponding maximum erosion rate value, i.e. $0.035 \mathrm{~mm}^{3} / \mathrm{g}$ (at $\sim 30^{\circ}$ incidence), $0.014 \mathrm{~mm}^{3} / \mathrm{g}$ (at $90^{\circ}$ incidence), and $0.116 \mathrm{~mm}^{3} / \mathrm{g}$ (at $90^{\circ}$ incidence) for the uncured $\mathrm{RM}$, cured RM, and borofloat glass, respectively. The borofloat glass and the cured mask materials exhibited typically brittle erosive behavior, while the uncured mask had a ductile erosive response. The most beneficial situation, therefore, is to perform AJM with the jet incident perpendicular to the surface, where the erosion is maximized for the target and cured mask, and minimized for the uncured mask. However, there are situations where use of an inclined jet is necessary, such as, e.g., when creating features with an abrupt change in depth [68]. In this case, curves such as Fig. 2.23 can be used to determine the relative erosion rates of the mask and substrate, so that the maximum achievable channel depth without undue mask erosion can be estimated. For the case of Fig. 2.23, in order to achieve machined features with geometries as close as possible to the desired pattern, it is essential to avoid machining with nozzle angles $<\sim 50^{\circ}$ where the uncured RM erosion rate increases drastically.

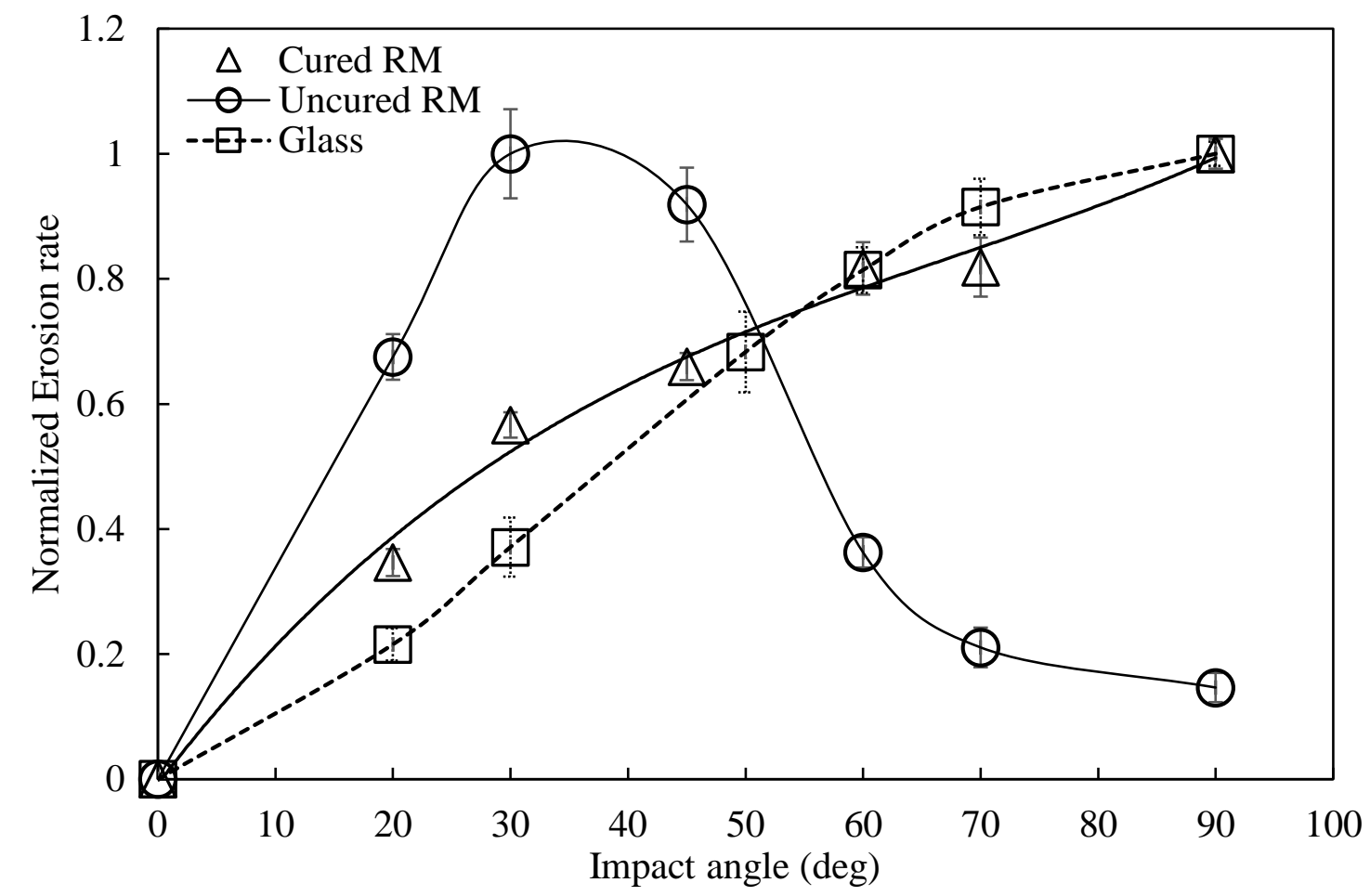

Figure 2.23- Normalized erosion rate of uncured, UV cured RM, and borosilicate glass as a function of angle of impingement. 90 indicates nozzle angle with respect to the RM surface. The curves were normalized to their maximum erosion rates of $0.035,0.014$, and $0.116 \mathrm{~mm}^{3} / \mathrm{g}$, for the UV cured RM, uncured RM, and glass, respectively. Error bars indicate the normalized standard deviation of 3 sets of experiments at each angle. 


\subsection{Summary}

The major conclusions of this chapter can be summarized as follows:

1) Rather than relying on manufacturer-specified curing times that are specific for a particular curing unit, it was found that measured energy density can be used to quantify a recommended cure that is independent of curing unit. For the tested configurations and irrespective of curing unit, the best pattern transfer from a designed pattern ultimately to eroded channels, was achieved using UV light energy densities between 516-774 and 387$516 \mathrm{~mJ} / \mathrm{cm}^{2}$, for the HT and HD RMs, respectively. This was roughly consistent with the curing times recommended by the manufacturer for their specific curing unit. Under these conditions, the width of eroded shallow channels were within approximately $3-9 \%$ of design.

2) The undercuring of RM resulted in wider than desired channels after AJM since a longer machining time was required to remove the cured RM regions. A small degree of overcuring the RM could be tolerated, although the features tended to get progressively larger as the energy densities increased above the recommended range.

3) When using the recommended energy density, the thinner HD RM resulted in a more accurate pattern transfer than the thicker HT masks, consistent with the claims of the manufacturer.

4) For the cylindrical UV exposure unit, it is recommended to cure the RM on the middle 20.5 $\mathrm{cm}$ along the length of the cylinder where the energy density was approximately constant for a given curing time.

5) As long as the recommended energy densities are used, the RMs can be cured from either side, dull or shiny, without loss of pattern transfer accuracy. This allows the user to adhere the mask onto the substrate surface before curing the RM.

6) A novel method to stack two RM layers was developed. As long as they are sufficiently cured (e.g. UV energy density $516-645 \mathrm{~mJ} / \mathrm{cm}^{2}$ for two layers of HT RM), eroded features 
with higher aspect ratios can be achieved by stacking the layers, without any loss in pattern transfer accuracy.

The information and findings of this chapter were implemented for fabrication of a 3D flow focusing microfluidic device using masked AJM (chapter 3). The parameters such as the amount of UV energy dose needed for multiple RM, Section 2.3.4, as well as the erosion rate of cured, uncured, and borosilicate glass, Section 2.3.6, were two of many findings that were used to fabricate the chip. 


\section{Application of AJM microfabrication to microfluidic three dimensional flow focusing}

The findings and results found in Chapter 2 were used to fabricate a 3D flow focusing device using AJM, as described in this chapter. The microfluidic chip with varying depth was made using double layer HD RM, with the results obtained from Section 2.3.4.

\subsection{Introduction}

Abrasive jet machining (AJM) has been used to fabricate microfluidic devices used for electrophoresis [47], electro-osmotic flow separation [25], and flow mixing [8]. However, AJM has not been applied to fabricate microfluidic chips with three-dimensional (3D) flow focusing capabilities. 3D focusing is the confinement of the sample flow toward the center of the flow cross section [10]. 3D flow focusing is possible by conventional soft lithography processes using polydimethylsiloxane (PDMS) [1]. To prevent microchannel clogging, it is desirable to have biological samples focused to the cross sectional center of the microchannel [1]. The most common technique to confine a sample flow is hydrodynamic focusing. It is usually done by introducing two sheath flows with higher flow rate from two sides of the sample flow.

Fabricating two dimensional microfluidic channels has been demonstrated using masked AJM techniques [25,48]. These studies used RapidMask to pattern desired geometry onto a substrate material, borosilicate glass, as explained in chapter 2. However, in both studies, the channels were limited to channels with constant depth along the length of the channels.

This chapter introduces the fabrication of a 3D flow focusing microfluidic device, where the depth of the channel is allowed to vary along the channel length, and controlled by the fabrication process combining RM and AJM. The information and results found in Chapters 1 and 2 were used to show the powerful application of RM in creating varying depth micro-channels using AJM. The fabricated chip was then tested with different flows to demonstrate flow focusing capabilities in both lateral and transverse direction in the channel.

\subsection{Experiments}

\subsubsection{Chip design}

The chip design was inspired by a design by Chiu et al. [1], Fig. 1.1. The design consisted of three inlets (one sample flow, two sheath flow) and one outlet. The original design was a combination of two layers (top and bottom) where very precise alignment was required. The new 
design only features microchannel patterns in a single layer, yet yields similar results to those by Chiu et al. [1]. This device has an increased channel depth, a slope of 30-60 at the flow focusing junction (where the three inlet flows meet), so that the sample flow is guided by the sheath flows for focusing of the suspension, Fig. 3.2. With this design, no alignment would be required after the fabrication of the chip. Alignment is typically a very delicate and time consuming process $[10,69,70]$. The full detail of the design is shown in Fig. 3.1 below. Channel A is the shallower channel where the sample flow enters the chip while channels B, C, D have the same center-line depths that are $100 \mu \mathrm{m}$ deeper than channel A.

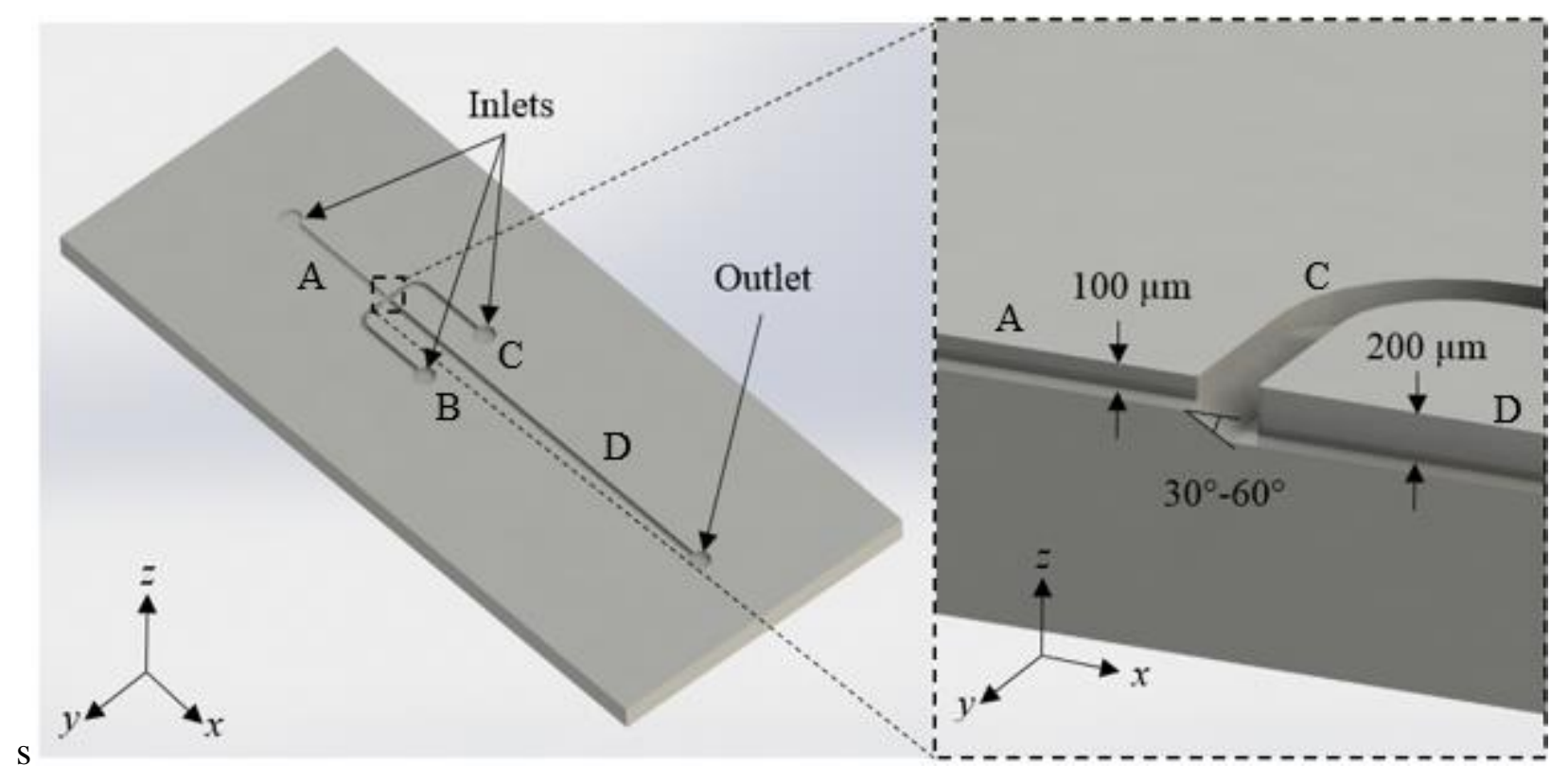

Figure 3.1- Schematic of the chip pattern design with $250 \mu \mathrm{m}$ channel width for all inlets channels and depth change at the junction. 


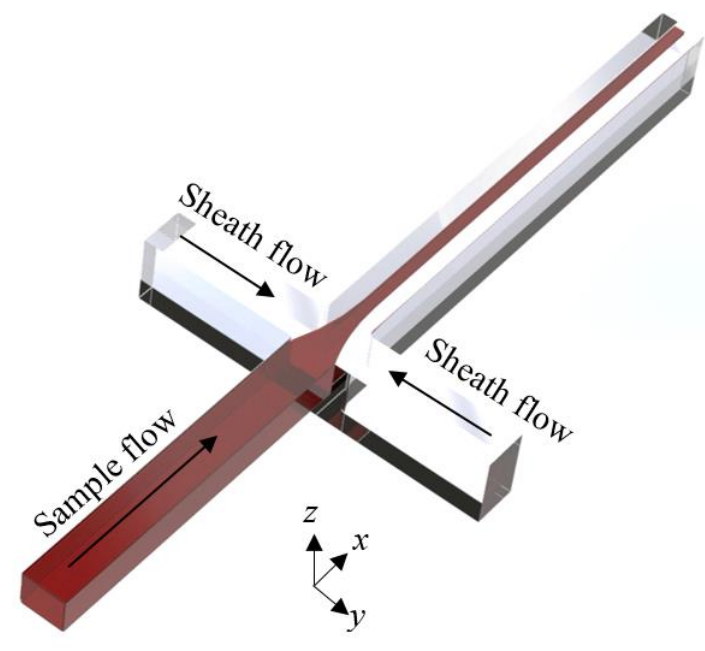

Figure 3.2 - 3D Schematic of the device as sample flow is focused in $y$ - $z$ plane by two sheath flows.

\subsubsection{Device fabrication}

The device was fabricated using double layers of HD RapidMask and AJM using the information and results obtained from Chapter 2. The microchannel was designed using computer aided design (CAD) software (AutoCAD 2016, Autodesk, Inc., Dan Rafael, CA, U.S.A.) and printed using a inkjet printer onto a transparency sheet (25,400 dpi, CAD/ART Services Inc., Bandon, OR, U.S.A.) to be used as photomasks. Figure 3.3 shows a schematic of the design used to fabricate the microfluidics chip. Two layers of HD RM were stacked together $(200 \mu \mathrm{m}$ thickness) before placing the patterned transparency sheet on them. The RM was then UV cured with a cylindrical UV exposure unit (Letralite, IKONICS Imaging, Duluth, MN, U.S.A.) with 516 $\mathrm{mJ} / \mathrm{cm}^{2} \mathrm{UV}$ energy density (Section 2.3.4). 


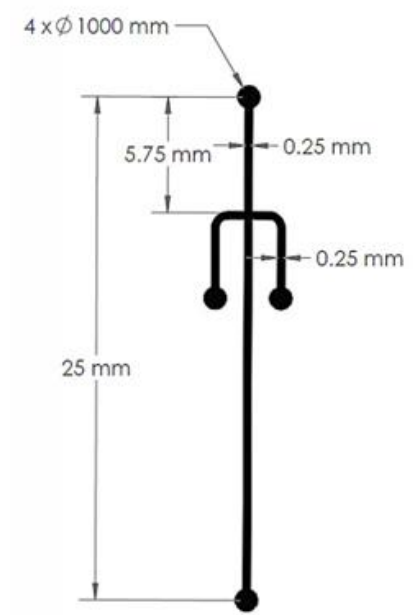

(a)

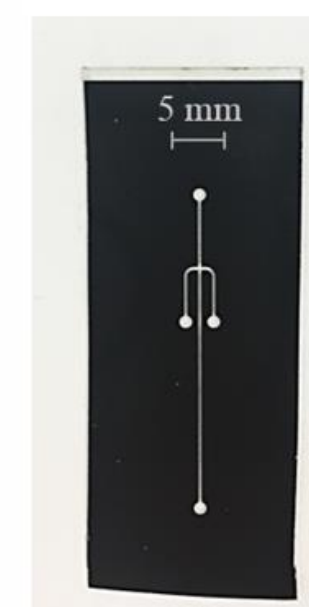

(b)

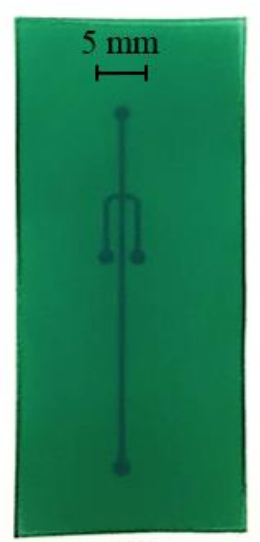

(c)

Figure 3.3- Schematic of (a) design layout (b) patterned transparency and (c) cured two layers HD RM used to fabricate the three dimensional flow focusing device.

The self-adhesive RM with the desired pattern was then placed onto a $1.1 \mathrm{~mm}$ thick borosilicate glass target (Borofloat, Swift glass Co. Inc., Elmira, NY, U.S.A.). The AJM blasting process was performed with equipment and input parameters explained in Section 2.2.2. An AccuFlo AF10 Micro-Abrasive Blaster, (Comco, Inc. Burbank, CA, U.S.A.) with $200 \mathrm{kPa}$ air pressure was used for the AJM process. A refrigeration air dryer, a dehumidifier, and a desiccant filter were implemented together to minimize the moisture in the experiment room that may lead to clogging of the abrasive jet tube [64]. To achieve a constant uniform particle mass flow rate, an electrical mixer (Arrow Model 850, Arrow Engineering Co., Inc., Hillside, NJ, U.S.A.) was placed in the reservoir. The abrasive particle used to fabricate the chip were Aluminum oxide $\left(\mathrm{Al}_{2} \mathrm{O}_{3}\right)$ powder (Comco Inc. Burbank, CA, U.S.A.) with nominal $25 \mu \mathrm{m}$ particle diameter.

The glass sample with cured RM with the desired pattern was clamped on a programmable computer controlled linear stage (Aerotech Inc., Pittsburgh, PA, U.S.A.). The stage was set to oscillate with $4 \mathrm{~mm}$ of amplitude, $8 \mathrm{~Hz}$ frequency at 10000 times cycles so that the entire design saw the same particle dose during the machining. The oscillating motion was set perpendicular to the scanning direction, and the target moved at a scanning velocity of $1 \mathrm{~mm} / \mathrm{sec}$ relative to the stationary nozzle, Fig. 3.4. The jet was fixed while the stage was controlled in two directions independently. A large 0.3 x 3.8 mm rectangular nozzle (Comco Inc. Burbank, CA, U.S.A.) was used for fabricating the chip with jet scanning direction parallel to the channel patterns on the RM. The nozzle standoff distance was kept at $20 \mathrm{~mm}$ from the surface of the sample. 


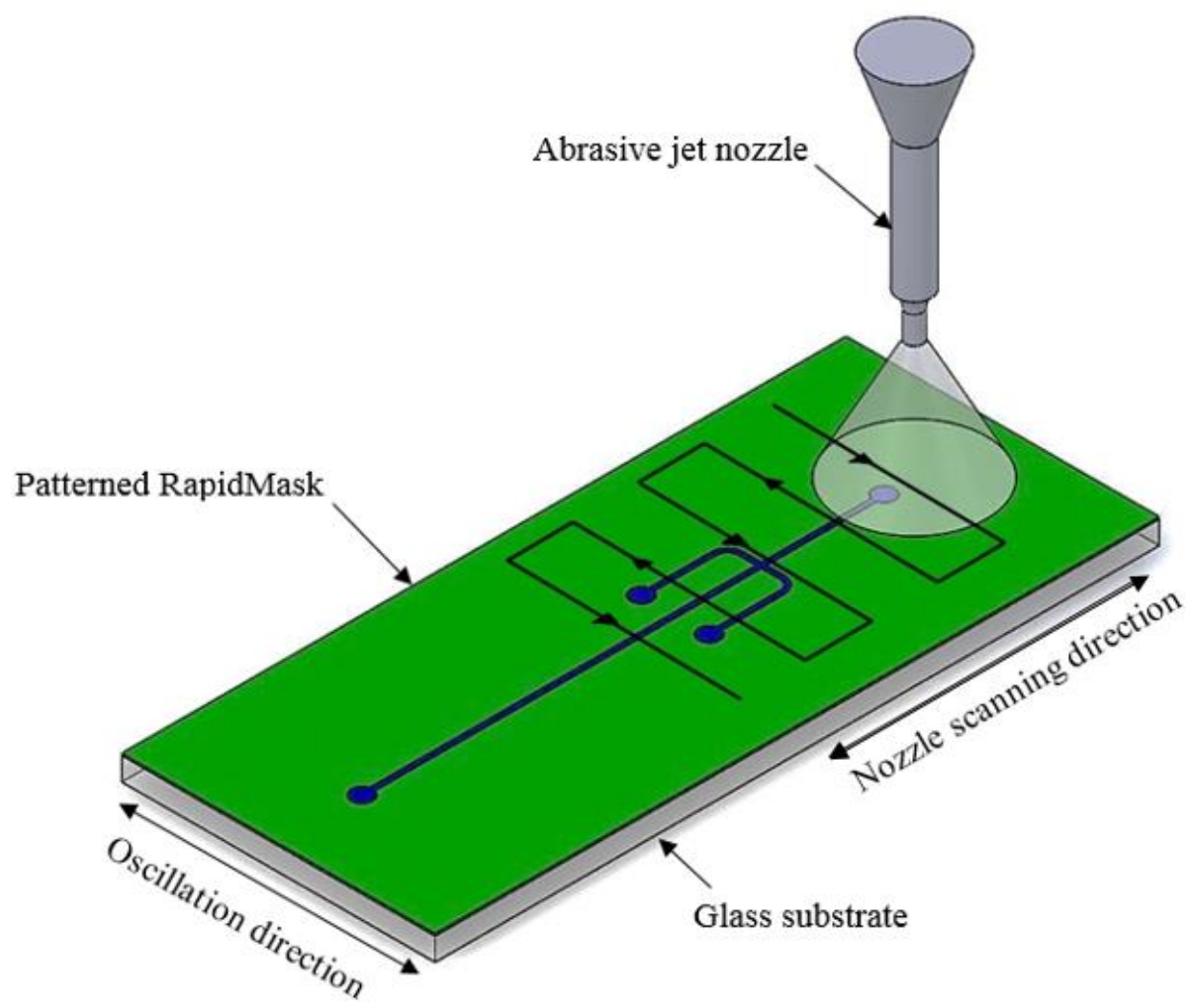

Figure 3.4 - Schematic of the procedure to etch the surface of the glass. The patterned RM is attached to the glass, and AJM is used to transfer the pattern to the glass substrate. Schematic is not scale.

The borosilicate glass with patterned RM was subjected to AJM with a $7.02 \mathrm{~g} / \mathrm{min}$ particle mass flow rate and a traverse speed of $1 \mathrm{~mm} / \mathrm{sec}$. The sample required ten passes to remove the cured (brittle) region (channel design). After the cured region was fully eroded, the sample was subjected to two more passes, creating a shallow channel on the glass surface. To create the slope at the junction, the sample flow inlet area of the design on RM was covered with a $300 \mu \mathrm{m}$ stainless steel shim. The shim was placed at the junction precisely using an optical microscope and image analysis (Clemex intelligent microscopy, Les Technologies Clemex Incorporated, Longueuil, Quebec, Canada). The sample was subjected to four more passes of machining, creating a deeper channel on the unprotected section of the target as well as a slope at the junction.

The RM was then removed and the chip was cleaned by high pressured air so that no particles were left in the eroded channels. Through thickness inlet holes were then machined using AJM with a $1 \mathrm{~mm}$ thick steel mask and multiple $1 \mathrm{~mm}$ diameter hole openings. The mask was fabricated using abrasive waterjet machining, OMAX 2626 Jet Machining Center (OMAX Corp., 
Kent, Washington, USA). The topographies of the eroded features were determined using an optical profilometer (NANOVEA ST400 Micro Photonics Inc., Irvine, CA, U.S.A.). The chip was then cleaned by ethanol using an ultrasonic cleaner (B-32 Ultrasonic Cleaner, Branson Ultrasonics, Danbury, CT, U.S.A.). After the fabricated chip was cleaned, it was bonded to another $1.1 \mathrm{~mm}$ thick borosilicate glass using a fusion bonding technique. The two glasses were placed together in an oven for 3 hours at $400{ }^{\circ} \mathrm{C}$ for the annealing process before the temperature was ramped up to $645^{\circ} \mathrm{C}$ for 6 hours during the thermal fusion of the two glasses.

A $35 \times 15 \times 4 \mathrm{~mm}$ slab of cured polydimethylsiloxane (PDMS) was used to seal the chip and for ease of attachment of the inlet/outlet pipette tips. The inlet and outlet holes were made using $1 \mathrm{~mm}$ diameter biopsy punch (Integra Miltex, Inc., Rietheim-Weilheim, Germany). After cleaning the PDMS and borosilicate glass, the two were irreversibly bonded using an oxygen plasma treatment (Harrick Plasma, Ithaca, NY, U.S.A.), Fig. 3.5.

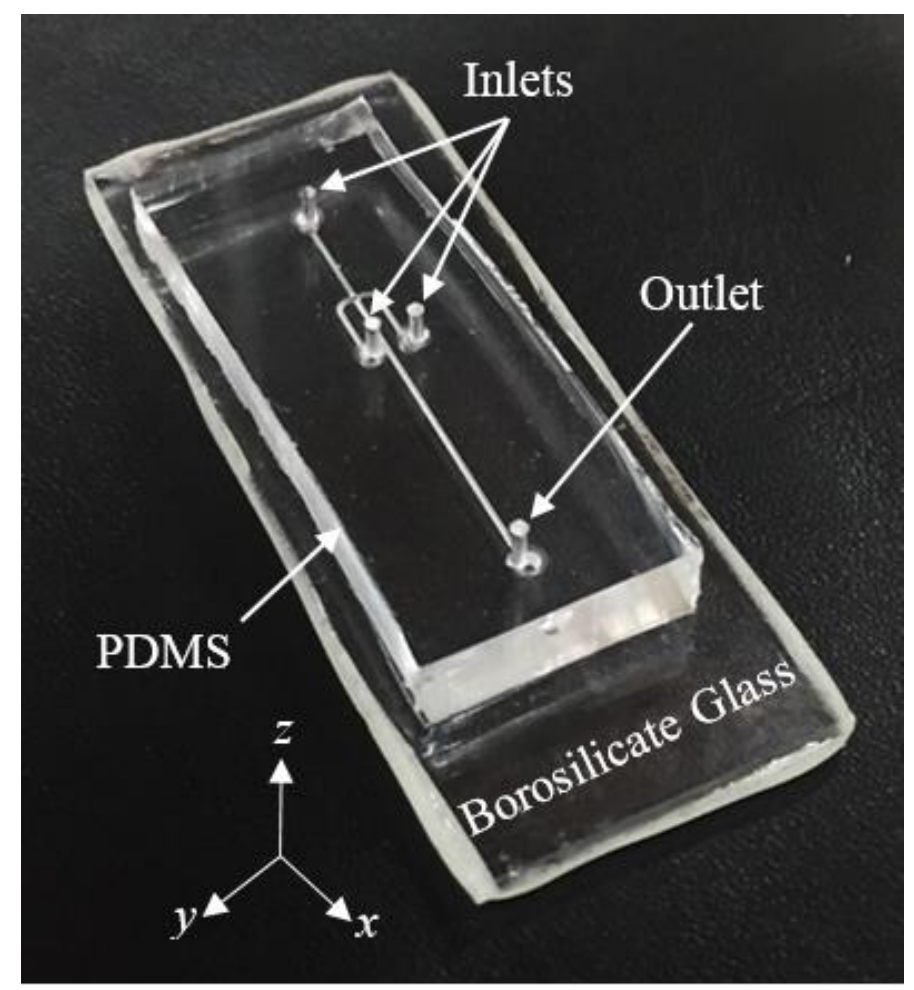

Figure 3.5 - Schematic of microfluidic chip fabricated on a borosilicate glass and PDMS layer on top. 


\subsubsection{Experimental set up for flow focusing application}

The flow focusing performance of the chip was tested by flowing black ink mixed with deionized (DI) water flowing from two sheath flow inlets. The pipette tips were inserted vertically into the inlets of the microfluidic device. The solutions were injected into the channel by a syringe pump (Harvard Apparatus, Holliston, MA, U.S.A.). The sample flow rate was set at $6 \mu \mathrm{L} / \mathrm{min}$ while the sheath flow rate was varied from 20 to $100 \mu \mathrm{L} / \mathrm{min}$ [1]. Images of flow focusing were captured using an inverted microscope (IX71, Olympus Corp., Tokyo, Japan) with 10× or 20× objectives (Fig. 3.6) and a high-speed camera (Miro M110, Vision Research, Wayne, NJ, U.S.A.) attached to the microscope.

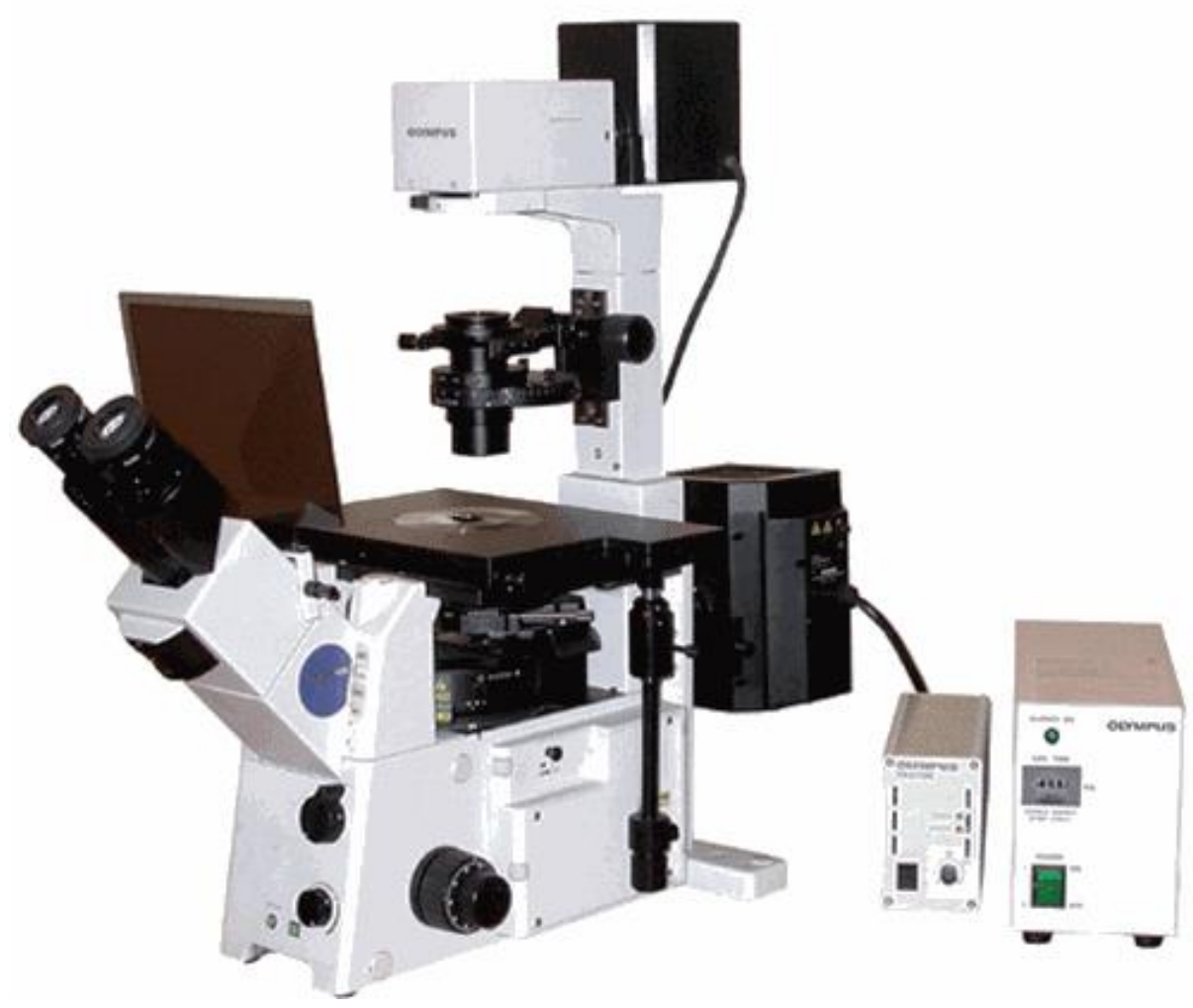

Figure 3.6 - A representative image of the inverted microscope set up used to image the flow focusing experiments [71].

Confocal imaging microscopy was performed to image the vertical position of the sample flow, to see if the sample flow was focused vertically. The focusing of the flow suspension was assessed using $0.1 \mu \mathrm{m}$ yellow-green fluorescent (505/515) FluoSpheres® Carboxylate-Modified Microspheres (Life technologies, Eugene, OR, U.S.A.) as sample flow and DI water for two sheath 
flows. The confocal images were captured using a Nikon A1 confocal microscope (Nikon Instruments Inc., Melville, NY, U.S.A.), Fig. 3.7. NIS-Elements Viewer advanced imaging software (Nikon Instruments Inc., Melville, NY, U.S.A.) was used for post-process of the images.

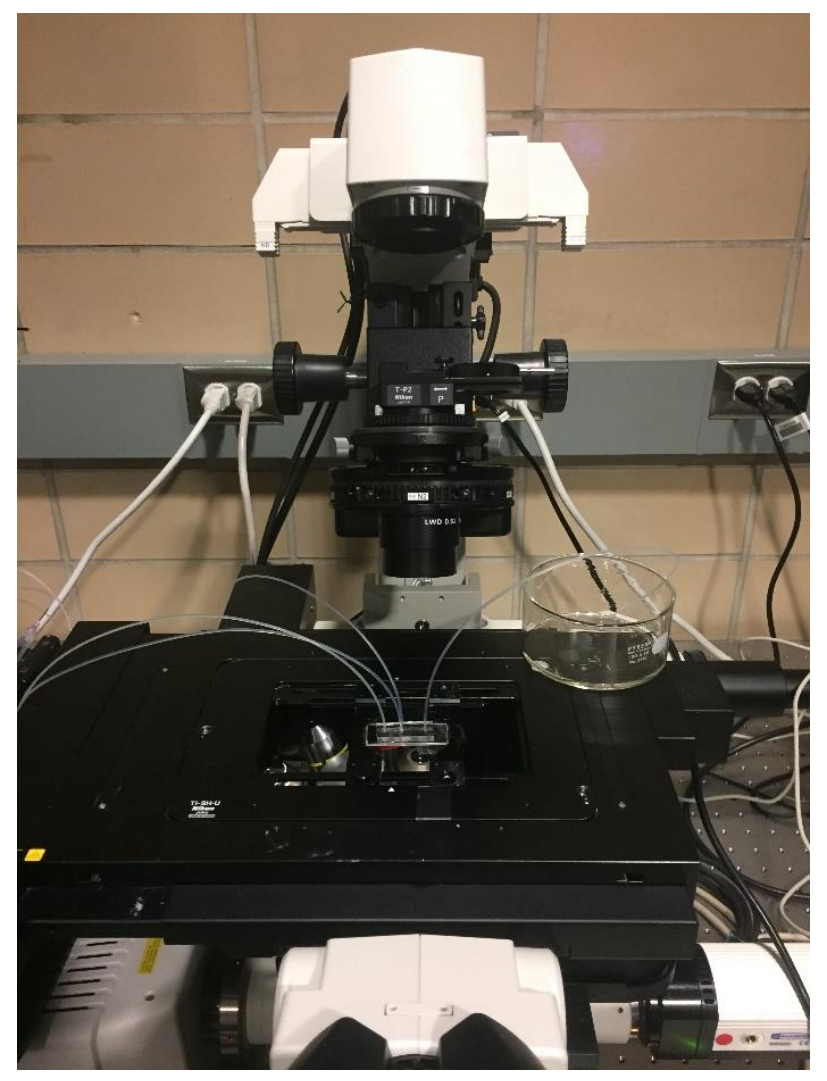

Figure 3.7 - A representative image of confocal imaging microscopy set up used to image the flow focusing experiments. 


\subsection{Results and Discussions}

\subsubsection{Shape of the fabricated chip}

Figure 3.8 shows the cross-sectional profile of the sheath flow and downstream channels, corresponding to channels B and C, and D, respectively, in Fig. 3.1. The measurements were performed near the flow focusing junction. Here, the downstream channel (D in Fig. 3.1) was designed to have greater depth than the two side sheath flow channels (B and C in Fig. 3.1).

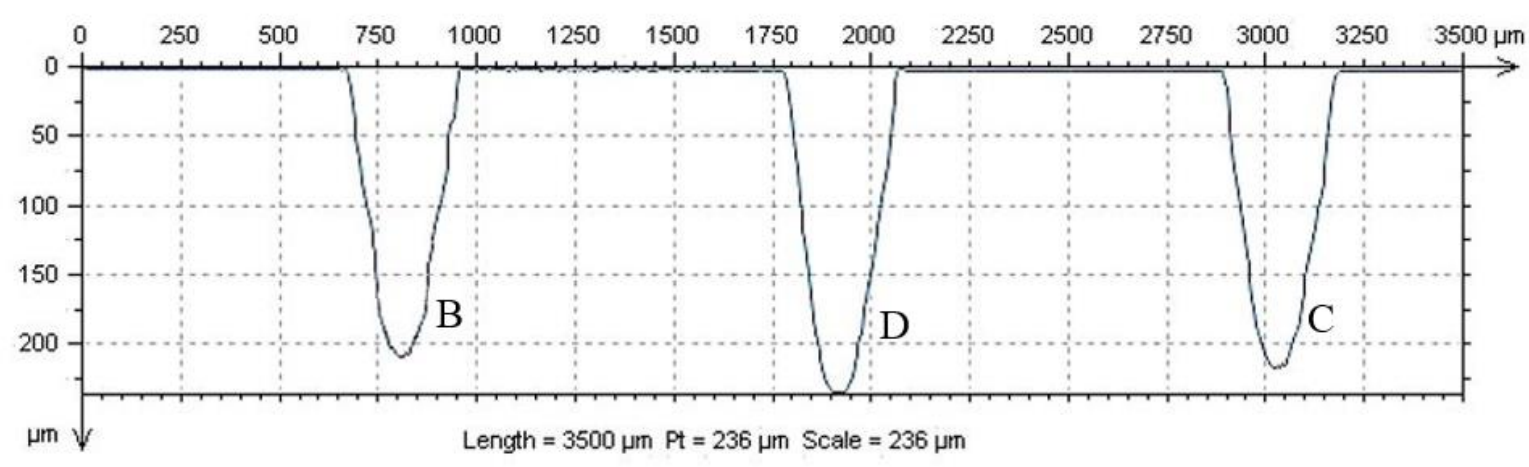

Figure 3.8 - Profile of channels B, C, D in Fig. 3.1 of the chip fabricated by AJM on a borosilicate substrate.

Figure 3.9 shows the width and center-line depth of all four channels of the fabricated chip on a borosilicate glass as well as the measured width of the cured RM channels. The average width of a cured RM channel was $263 \mu \mathrm{m}$ for all channels. The average width of the eroded shallow channel, channel A, was $265 \mu \mathrm{m}$ which was machined with two passes at $1 \mathrm{~mm} / \mathrm{sec}$ transverse speed. The measured width of Channels B, C, and D were 287, 289, and $290 \mu \mathrm{m}$, respectively. These results were expected since channels B, C, and D were fabricated using a much higher particle dose, i.e. six passes at $1 \mathrm{~mm} / \mathrm{sec}$ transverse speed.

Figure 3.9 shows the resulting average measured centerline depth of the eroded channel. The average center-line depth of channel A was $120 \mu \mathrm{m}$. The depth of channel D was measured to be $225 \mu \mathrm{m}$ while channels B and C average center-line depths were 215 and $220 \mu \mathrm{m}$, respectively. The two sheath flows depths were slightly shallower than the sample flow channel because during the AJM process, channel D saw a higher particle dose per oscillation than the other channels. The measured slope at the junction was found to be $33^{\circ}$. 


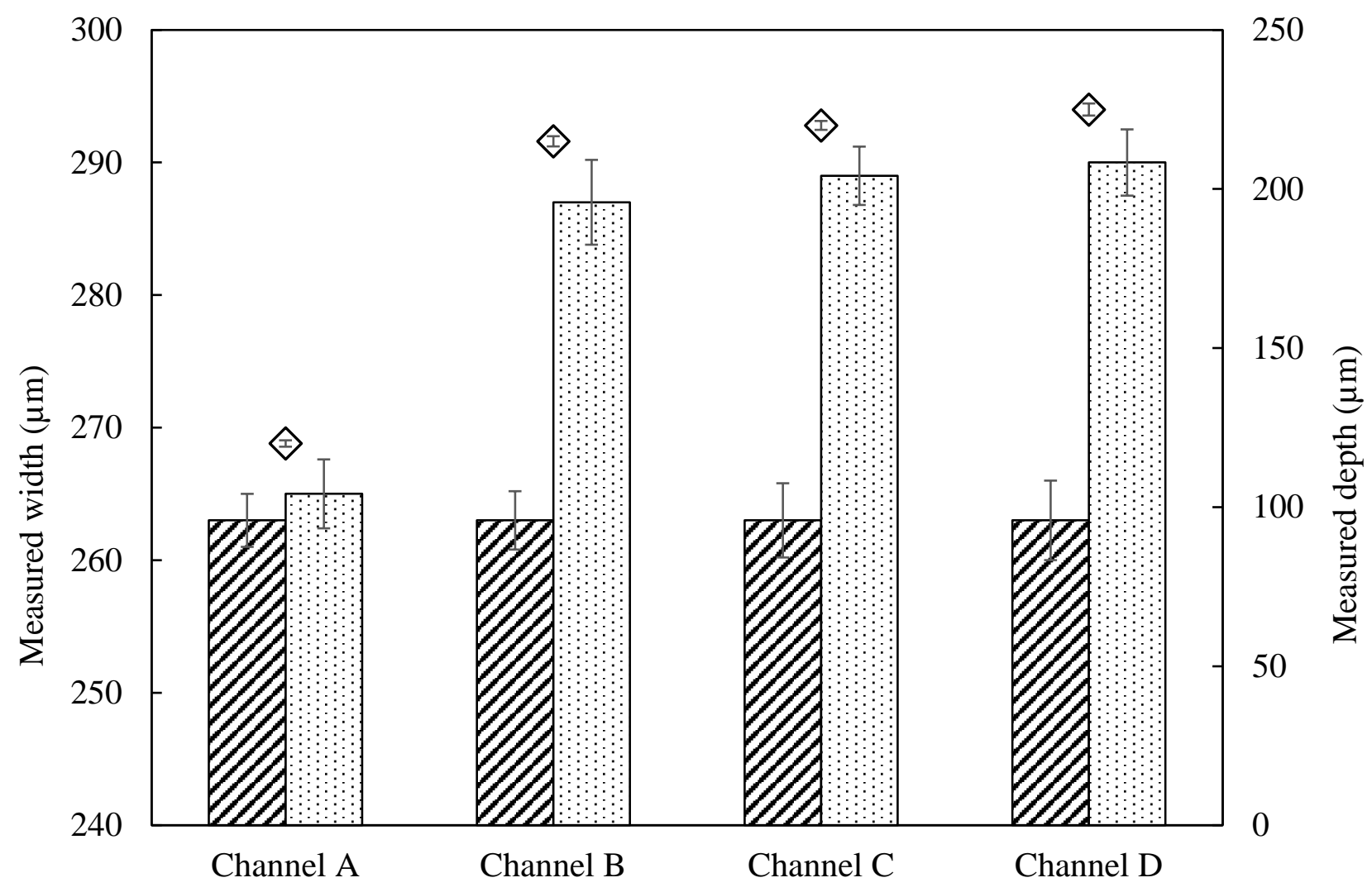

$\square$ Width of cured RM channel $\square$ Width of AJM channel $\diamond$ Centre-line depth of AJM channel

Figure 3.9 - Measured width of cured RM and AJM channels of A, B, C, D channels, Fig. 3.1, shown in primary axis with measured depth of the channels shown on the secondary axis. The error bars indicate one standard deviation of 3 measurements of RM and eroded channels.

\subsubsection{Three-dimensional flow focusing}

Figure 3.10 shows the focusing of the sample flow in the lateral $(y)$ direction. The flow rate of the sample flow and the two sheath flows were at 6 and $100 \mu \mathrm{L} / \mathrm{min}$, respectively. This experimental image shows that the sample was hydrodynamically focused in the lateral direction of the chip. The sample flow was focused from $265 \mu \mathrm{m}$ wide to approximately $10 \mu \mathrm{m}$ wide stream, i.e. the sample flow was focused $96 \%$ in lateral direction. 


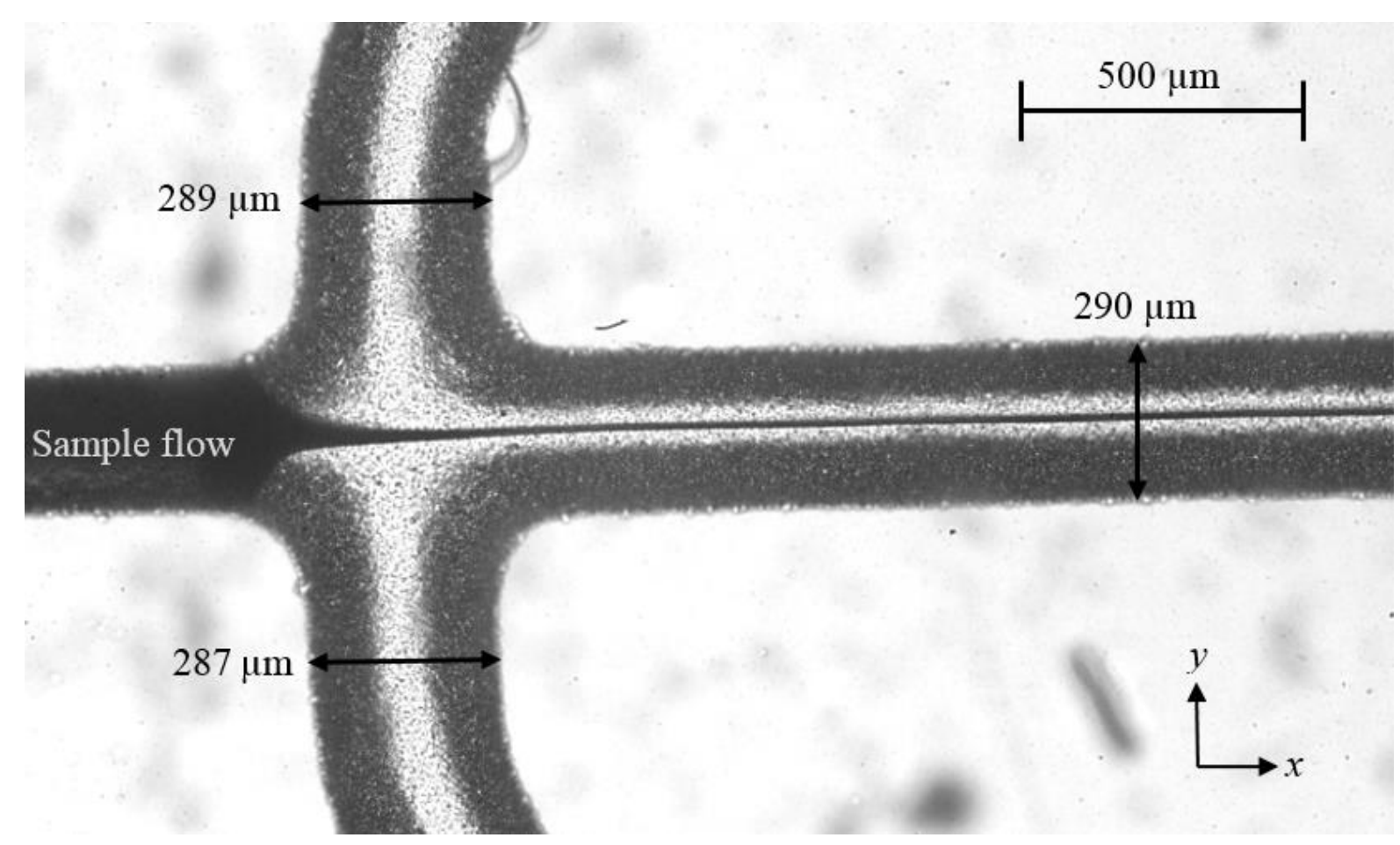

Figure 3.10 - An image of top view of a focused flow in the microfluidic chip fabricated with AJM using RM. The flow rate was set at $6 \mu \mathrm{L} / \mathrm{min}$ and $100 \mu \mathrm{L} / \mathrm{min}$ for sample and two sheath flows, respectively.

Figure 3.11 shows a confocal image of the microfluidic chip and the focusing of the flow in lateral direction as well as the suspension. The flow rate of the sample flow and the two sheath flows were at 6 and $100 \mu \mathrm{L} / \mathrm{min}$, respectively. The flow was pushed down $\sim 50 \mu \mathrm{m}$ in negative $z$ direction by the two sheath flows immediately downstream of the focusing junction, Fig. 3.11. The flow was moved downward for another $\sim 50 \mu \mathrm{m}$ at $\sim 2 \mathrm{~cm}$ downstream of the focusing junction. However, there was evidence of the sample flow at the bottom of the channel, Fig. 3.11. It is hypothesized that this secondary-focused flow was due to the different depths of the downstream channel and two sheath flow channels, described in Section 3.3.1. The depth of the sheath flow channels, channel B and C, were slightly less than the depth of the main downstream channel, channel D, Fig. 3.9. The sample flow at the bottom of the main downstream channel was not focused to the center of the channel by the sheath flows because the sample flow entered the downstream channel at a depth of $225 \mu \mathrm{m}$, which is deeper than the sheath flow channels, 215 and $220 \mu \mathrm{m}$. This effect can be mitigated by making the sample flow channel and sheath flow channels such that they have the same depth. In addition, it was anticipated that if the sheath flow channels were slightly deeper than the main downstream channel, the sheath flows would affect the sample 
flow entirely in the similar manner as if all channels have equal depths. These ideas will be implemented in the next prototype of the chip.

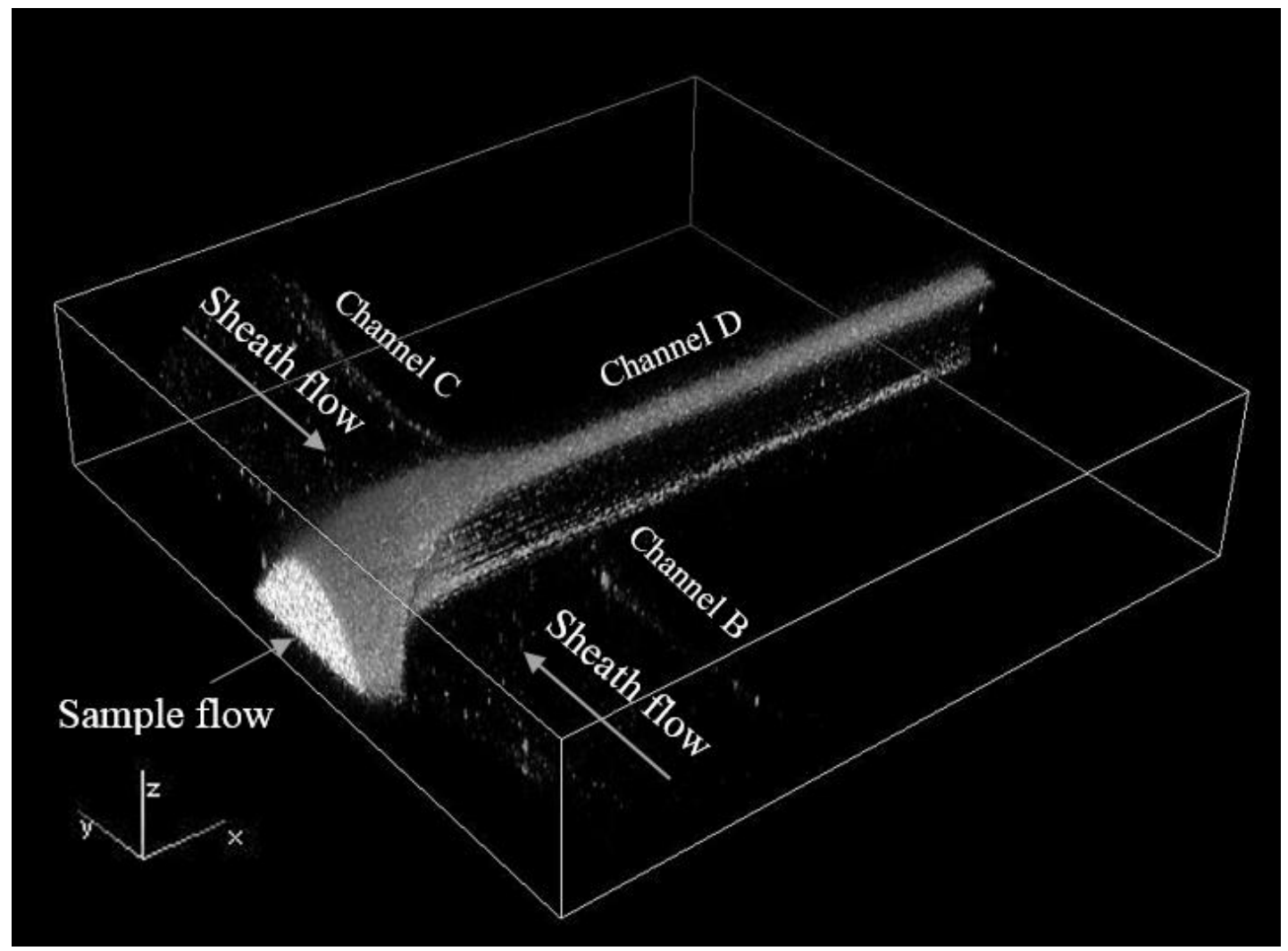

Figure 3.11 - The confocal imaging of the channel at the focusing junction. The flow rate was set at $6 \mu \mathrm{L} / \mathrm{min}$ and $100 \mu \mathrm{L} / \mathrm{min}$ for sample flow rate and two sheath flows, respectively.

\subsection{Summary}

A 3D dimensional flow focusing microfluidic chip was fabricated using AJM on a glass substrate. The device was made using double layer HD RM and the results found for RM during the AJM process. The flow focusing chip with varying depth at the junction was then fusion bonded to another glass slide and tested experimentally. The sample flow was focused in lateral direction by two sheath flows, Fig. 3.10. Using confocal imaging, the focusing of the flow suspension in the vertical was demonstrated. However, it was found that there was an unanticipated flow at the bottom of the channel which might be eliminated in future work by ensuring that the depths of the sample and sheath flow channels, are closer to equal. 


\section{Conclusions and Future Work}

\subsection{Conclusion}

The principle conclusions of this thesis can be summarized as follows:

(i) Instead of using the manufacturer-specified curing times that are specific for a particular curing unit, it was found that measured energy density can be used to quantify a recommended cure time and light intensity that is independent of curing unit. For the tested configurations and irrespective of curing unit, the best pattern transfer from a designed pattern ultimately used to erode channels, was achieved using UV light energy densities between 516-774 and $387-516 \mathrm{~mJ} / \mathrm{cm}^{2}$, for the HT and HD RMs, respectively. This was roughly consistent with the curing intensity recommended by the manufacturer for their specific curing unit. Under these conditions, the width of eroded shallow channels were within approximately 3-9\% of design.

(ii) The undercuring of RM resulted in wider than desired channels after AJM since a longer machining time was required to remove the cured RM regions. A small degree of overcuring of RM could be tolerated. In addition, when RM was cured above the recommended range of UV energy density, the features tended to become progressively larger.

(iii) For the cylindrical UV exposure unit, it is recommended to cure the RM on the middle $20.5 \mathrm{~cm}$ along the length of the cylinder where the energy density is approximately constant for a given curing time.

(iv) The RM mask can be cured through either of its sides, dull or shiny, achieving the same pattern transfer accuracy. Therefore, masks can be adhered to the substrate before curing them.

(v) A novel method to stack two RM layers was developed. As long as they are sufficiently cured (e.g. UV energy density $516-645 \mathrm{~mJ} / \mathrm{cm}^{2}$ for two layers of HT 
RM), eroded features with higher aspect ratios can be achieved by stacking the layers, without any loss in pattern transfer accuracy.

(vi) A 3D flow focusing device was fabricated using double layer HD RM and AJM. The channel depth change at the junction allowed control of the location of the focused flow, in the vertical direction, in addition to the lateral direction. 


\subsection{Recommendations for future work}

The research presented in this thesis could be extended to study the following other interesting aspects of RapidMask used in AJM and microfluidic chip fabrication.

(i) More than two layers of RM in AJM can be used to achieve higher aspect ratio channels on glass. The multiple layered (more than two) masks increase the erosion resistance of the RM. Sufficient amount of UV energy density to cure more than two layers of RM needs to be studied before investigating their performance during the AJM process.

(ii) A steeper slope at the junction can be achieved with AJM nozzle inclined, Section 3.2.1 and 3.3.1. This could be accomplished by using the information given in Section 2.3.6 to machine the maximum achievable slope with geometries as close as possible to the desired pattern.

(iii) The fusion bonding of the fabricated chip can be eliminated if the PDMS used for placing pipette tips is also used for sealing the channel on the borosilicate glass instead of another glass slide, Section 3.2.2. This is achievable if the inlets/outlet holes on the chip are not through-holes. With this configuration, the PDMS slap can be used to seal the channel and also used for its initial purpose of inserting the inlets/outlet pipette tips.

(iv) The chip can be improved by ensuring that the depths of the sample and sheath flow channels, are closer to equal. It is anticipated that equal or slightly deeper sheath flow channels will eliminate any unexpected flow seen on the bottom of the channel, shown in Fig. 3.9. 


\section{References}

[1] Chiu Y, Cho SH, Mei Z, Lien V, Wu T, Lo Y. Universally applicable three-dimensional hydrodynamic microfluidic flow focusing. Lab Chip 2013;13:1803-9. doi:10.1039/c3lc41202d.

[2] van Dijke K, Veldhuis G, Schroën K, Boom R. Parallelized edge-based droplet generation (EDGE) devices. Lab Chip 2009;9:2824. doi:10.1039/b906098g.

[3] Roederer M, Brenchley JM, Betts MR, De Rosa SC. Flow cytometric analysis of vaccine responses: How many colors are enough? Clin Immunol 2004;110:199-205. doi:10.1016/j.clim.2003.11.015.

[4] Jennings CD, Foon KA. Flow cytometry: recent advances in diagnosis and monitoring leukemia. Cancer Investig 1997;15:384-99.

[5] Lehmann AK, Sørnes S, Halstensen A. Phagocytosis: Measurement by flow cytometry. J Immunol Methods 2000;243:229-42. doi:10.1016/S0022-1759(00)00237-4.

[6] Keeney M, Chin-Yee I, Weir K, Popma J, Nayar R, Robert Sutherland D. Single platform flow cytometric absolute CD34+ cell counts based on the ISHAGE guidelines. Commun Clin Cytom 1998;34:61-70.

[7] Jun Y, Kim MJ, Hwang YH, Jeon EA, Kang AR, Lee SH, et al. Microfluidics-generated pancreatic islet microfibers for enhanced immunoprotection. Biomaterials 2013;34:812230. doi:10.1016/j.biomaterials.2013.07.079.

[8] Sayah A, Thivolle P-A, Parashar VK, Gijs M a M. Fabrication of microfluidic mixers with varying topography in glass using the powder-blasting process. J Micromechanics Microengineering 2009;19:85024. doi:10.1088/0960-1317/19/8/085024.

[9] Anderson JR, Chiu DT, Jackman RJ, Chemiavskaya O, McDonald JC, Wu H, et al. Fabrication of topologically complex three-dimensional microfluidic systems in PDMS by rapid prototyping. Anal Chem 2000;72:3158-64. doi:10.1021/ac9912294.

[10] Lu M, Ozcelik A, Grigsby CL, Zhao Y, Guo F, Leong KW, et al. Microfluidic hydrodynamic focusing for synthesis of nanomaterials. Nano Today 2016. doi:http://dx.doi.org/10.1016/j.nantod.2016.10.006.

[11] Li X, Liu X. Fabrication of three-dimensional microfluidic channels in a single layer of cellulose paper. Microfluid Nanofluidics 2014;16:819-27. doi:10.1007/s10404-014-1340- 
Z.

[12] Martinez AW, Phillips ST, Whitesides GM. Three-dimensional microfluidic devices fabricated in layered paper and tape. Proc Natl Acad Sci U S A 2008;105:19606-11. doi:10.1073/pnas.0810903105.

[13] Ghobeity A, Krajac T, Burzynski T, Papini M, Spelt JK. Surface evolution models in abrasive jet micromachining. Wear 2008;264:185-98. doi:10.1016/j.wear.2007.02.020.

[14] Belloy E, Pawlowski AG, Sayah A, Gijs MAM. Microfabrication of high-aspect ratio and complex monolithic structures in glass. J Microelectromechanical Syst 2002;11:521-7. doi:10.1109/JMEMS.2002.803418.

[15] Ghobeity A, Ciampini D, Papini M. An analytical model of the effect of particle size distribution on the surface profile evolution in abrasive jet micromachining. J Mater Process Technol 2009;209:6067-77. doi:10.1016/j.jmatprotec.2009.05.026.

[16] Slikkerveer PJ, Veld FHI. Model for patterned erosion. Wear 1999;233-235:377-86. doi:10.1016/S0043-1648(99)00177-5.

[17] Nouhi A, Sookhak Lari MR, Spelt JK, Papini M. Implementation of a shadow mask for direct writing in abrasive jet micro-machining. J Mater Process Technol 2015;223:232-9. doi:10.1016/j.jmatprotec.2015.04.007.

[18] Iliescu C, Chen B, Miao J. On the wet etching of Pyrex glass. Sensors Actuators, A Phys 2008;143:154-61. doi:10.1016/j.sna.2007.11.022.

[19] Cheng X, Wang Z, Nakamoto K, Yamazaki K. A study on the micro tooling for micro/nano milling. Int J Adv Manuf Technol 2011;53:523-33. doi:10.1007/s00170-010-2856-3.

[20] Liu HT, Sun YZ, Shan DB, Zhang CX. Experimental research of brittle-ductile transition conditions and tool wear for micromilling of glass material. Int J Adv Manuf Technol 2013;68:1901-9. doi:10.1007/s00170-013-4987-9.

[21] Zheng Z-P, Lin J-K, Huang F-Y, Yan B-H. Improving the machining efficiency in electrochemical discharge machining (ECDM) microhole drilling by offset pulse voltage. J Micromechanics Microengineering 2008;18:25014. doi:10.1088/0960-1317/18/2/025014.

[22] Kucukturk G, Can C. A New Method for Machining of Electrically Nonconductive Workpieces Using Electric Discharge Machining. Mach Sci Technol 2010;14:189-207. doi:10.1080/10910344.2010.500497. 
[23] Ogura H, Yoshida Y. Hole Drilling of Glass Substrates with a CO 2 Laser. Jpn J Appl Phys 2003;42:2881-6. doi:10.1143/JJAP.42.2881.

[24] Wang J, Nguyen T, Pang KL. Mechanisms of microhole formation on glasses by an abrasive slurry jet. J Appl Phys 2009;105. doi:10.1063/1.3079802.

[25] Ghobeity A, Crabtree HJ, Papini M, Spelt JK. Characterisation and comparison of microfluidic chips formed using abrasive jet micromachining and wet etching. J Micromechanics Microengineering 2012;22:25014. doi:10.1088/0960-1317/22/2/025014.

[26] Burzynski T, Papini M. Analytical model of particle interference effects in divergent erosive jets. Tribol Int 2010;43:554-67. doi:10.1016/j.triboint.2009.09.004.

[27] Zhang L, Kuriyagawa T, Yasutomi Y, Zhao J. Investigation into micro abrasive intermittent jet machining. Int J Mach Tools Manuf 2005;45:873-9. doi:10.1016/j.ijmachtools.2004.11.003.

[28] Wensink H, Schlautmann S, Goedbloed MH, Elwenspoek MC. Fine tuning the roughness of powder blasted surfaces. J Micromechanics Microengineering 2002;12:616-20. doi:10.1088/0960-1317/12/5/316.

[29] Mohammad Jafar RH, Spelt JK, Papini M. Surface roughness and erosion rate of abrasive jet micro-machined channels: Experiments and analytical model. Wear 2013;303:302-12. doi:10.1016/j.wear.2013.03.005.

[30] Hutchings IM. Ductile-brittle transitions and wear maps for the erosion and abrasion of brittle materials. J Phys D Appl Phys 1992;25:A212-21. doi:10.1088/00223727/25/1A/033.

[31] Sheikh-Ahmad JY. Machining of Polymer Composites. Abu Dhabi: Springer; 2009. doi:10.1007/978-0-387-68619-6.

[32] Finnie I. Some Observations on the Erosion of Ductile Metals. Wear 1972;19:81-90.

[33] Tilly GP. A two stage mechanism of ductile erosion. Wear 1973;23:87-96. doi:10.1016/0043-1648(73)90044-6.

[34] Hutchings IM, Winter RE. Particle erosion of ductile metals: A mechanism of material removal. Wear 1974;27:121-8. doi:10.1016/0043-1648(74)90091-X.

[35] Papini M, Dhar S. Experimental verification of a model of erosion due to the impact of rigid single angular particles on fully plastic targets. Int J Mech Sci 2006;48:469-82. 
doi:10.1016/j.ijmecsci.2005.12.010.

[36] Tilly GP, Sage S. The interaction of particle and material bahaviour in erosion processes. Wear 1970;16:447-65.

[37] Sheldon GL, Finnie I. On the Ductile Behavior of Nominally Brittle Materials During Erosive Cutting. J Eng Ind 1966;88:387-92.

[38] Misra A, Finnie I. On the size effect in abrasive and erosive wear. Wear 1981;65:359-73. doi:10.1016/0043-1648(81)90062-4.

[39] Barkoula NM, Karger-Kocsis J. Processes and influencing parameters of the solid particle erosion of polymers and their composites. J Mater Sci 2002;37:3807-20. doi:10.1023/A:1019633515481.

[40] Ali YM, Mathew P, Wang J. Progress in the Modeling of Abrasive Jet Machining. Adv Mater Res 2010;126-128:3-8. doi:10.4028/www.scientific.net/AMR.126-128.3.

[41] Ali YM, Wang J. Machining with abrasives. Springer; 2011. doi:10.1007/978-1-4419-73023.

[42] Aquaro D, Fontani E. Erosion of ductile and brittle materials. Meccanica 2001;36:651-61. doi:10.1023/A:1016396719711.

[43] Verspui MA, De With G, Corbijn A, Slikkerveer PJ. Simulation model for the erosion of brittle materials. Wear 1999;233-235:436-43. doi:10.1016/S0043-1648(99)00180-5.

[44] Mohammad Jafar RH, Spelt JK, Papini M. Numerical simulation of surface roughness and erosion rate of abrasive jet micro-machined channels. Wear 2013;303:302-12. doi:10.1016/j.wear.2013.03.021.

[45] Basak AK, Fan JM, Wang J, Mathew P. Material removal mechanisms of monocrystalline silicon under the impact of high velocity micro-particles. Wear 2010;269:269-77. doi:10.1016/j.wear.2010.04.006.

[46] Zeng, J. Kim T. An erosion model of polycrystalline ceramic in abrasive water jet cutting. Wear 1996;193:275-82.

[47] Solignac D, Sayah A, Constantin S, Freitag R, Gijs MAM. Powder blasting for the realisation of microchips for bio-analytic applications. Sensors Actuators, A Phys 2001;92:388-93. doi:10.1016/S0924-4247(01)00577-5.

[48] Burzynski T, Papini M. Modelling surface evolution in abrasive jet micromachining using 
level se. Ryerson University, 2012.

[49] Schlautmann S, Wensink H, Schasfoort R, Elwenspoek M, Berg A Van Den. Powderblasting technology as an alternative tool for microfabrication of capillary electrophoresis chips with integrated conductivity sensors. J Micromechanics Microengineering 2001;11:386-9. doi:10.1088/0960-1317/11/4/318.

[50] Belloy E, Sayah A, Gijs MAM. Micromachining of glass inertial sensors. J Microelectromechanical Syst 2002;11:85-90. doi:10.1109/84.982867.

[51] Park DS, Cho MW, Seo TI. Mechanical etching of micro pockets by powder blasting. Int J Adv Manuf Technol 2005;25:1098-104. doi:10.1007/s00170-003-1941-2.

[52] Yamahata C, Lacharme F, Burri Y, Gijs MAM. A ball valve micropump in glass fabricated by powder blasting. Sensors Actuators, B Chem 2005;110:1-7. doi:10.1016/j.snb.2005.01.005.

[53] Wensink H, Jansen H V, Berenschot JW, Elwenspoek MC. Mask materials for powder blasting. J Micromechanics Microengineering 2000;10:175-80. doi:10.1088/0960$1317 / 10 / 2 / 313$.

[54] Liao YS, Chen LC. A method of etching and powder blasting for microholes on brittle $\begin{array}{llll}\text { materials. } & \text { J } & \text { Mater 2009;209:4390-4 }\end{array}$ doi:10.1016/j.jmatprotec.2008.11.032.

[55] Pawlowski AG, Sayah A, Gijs MAM. Precision poly-(dimethyl siloxane) masking technology for high-resolution powder blasting. J Microelectromechanical Syst 2005;14:619-24. doi:10.1109/JMEMS.2005.844745.

[56] Lomas T, Wisitsoraat a, Chevasuvit F, Tuantranont a. A precision hot embossing mold fabricated by high-resolution powder blasting with polydimethylsiloxane and SU-8 masking technology. J Micromechanics Microengineering 2009;19:35002. doi:10.1088/0960-1317/19/3/035002.

[57] Park DS, Cho MW, Lee H, Cho WS. Micro-grooving of glass using micro-abrasive jet $\begin{array}{llll}\text { machining. } & \text { J } & \text { Mater } & \text { Process }\end{array}$ Technol 146:234-40. doi:10.1016/j.jmatprotec.2003.11.013.

[58] Yagyu H, Sugano K, Hayashi S, Tabata O. Micropowder blasting with nanoparticles dispersed polymer mask for rapid prototyping of glass chip. J Micromechanics 
Microengineering 2005;15:1236-41. doi:10.1088/0960-1317/15/6/014.

[59] Slikkerveer PJ, Touwslager FJ. Erosion of elastomeric protective coatings. Wear 1999;236:189-98. doi:10.1016/S0043-1648(99)00268-9.

[60] Achtsnick M, Drabbe J, Hoogstrate AM, Karpuschewski B. Erosion behaviour and pattern transfer accuracy of protecting masks for micro-abrasive blasting. J Mater Process Technol 2004;149:43-9. doi:10.1016/j.jmatprotec.2003.10.037.

[61] RapidMask High Detail 2015:2.

[62] RapidMask High Tack 2015:2.

[63] Safety Data Sheet-Rapid Mask flims 2006:1-11.

[64] Ghobeity A, Getu H, Krajac T, Spelt JK, Papini M. Process repeatability in abrasive jet micro-machining. J Mater Process Technol 2007;190:51-60. doi:10.1016/j.jmatprotec.2007.03.111.

[65] Getu H, Ghobeity A, Spelt JK, Papini M. Abrasive jet micromachining of polymethylmethacrylate. Wear 2007;263:1008-15. doi:10.1016/j.wear.2007.01.063.

[66] Li HZ, Wang J, Fan JM. Analysis and modelling of particle velocities in micro-abrasive air jet. Int J Mach Tools Manuf 2009;49:850-8. doi:10.1016/j.ijmachtools.2009.05.012.

[67] Ghobeity A, Papini M, Spelt JK. Computer simulation of particle interference in abrasive jet micromachining. Wear 2007;263:265-9. doi:10.1016/j.wear.2007.01.112.

[68] Ghobeity A, Papini M, Spelt JK. Abrasive jet micro-machining of planar areas and transitional slopes in glass using target oscillation. J Mater Process Technol 2009;209:5123-32. doi:10.1016/j.jmatprotec.2009.02.012.

[69] Love JC, Wolfe DB, Jacobs HO, Whitesides GM. Microscope projection photolithography for rapid prototyping of masters with micron-scale features for use in soft lithography. Langmuir 2001;17:6005-12. doi:10.1021/la010655t.

[70] Stephan K, Pittet P, Renaud L, Kleimann P, Morin P, Ouaini N, et al. Fast prototyping using a dry film photoresist: microfabrication of soft-lithography masters for microfluidic structures. J Micromechanics Microengineering 2007;17:N69-74. doi:10.1088/0960$1317 / 17 / 10 / \mathrm{N} 01$.

[71] Olympus. Olympus IX71 inverted microscopes 2015:30. 